\title{
CAPACITY, ENERGY AND POTENTIAL THEORY FOR RANDOM FIELDS
}

\begin{abstract}
MING YANG
Abstract. Let $X: \mathbb{R}^{N} \rightarrow \mathbb{R}^{d}$ be a random field. We define capacity and energy and obtain a two-sided inequality relating capacity and energy for $X$. We apply our potential-theoretic results to various hitting probabilities for Markov fields. For non-Markovian fields, similar hitting probability results will be given elsewhere.
\end{abstract}

\section{INTRODUCTION}

Let $X: \mathbb{R}^{N} \rightarrow \mathbb{R}^{d}$ be a field having the paths which are right continuous with left limits (rcll) in the usual partial order $\preceq$ of $\mathbb{R}^{N}: s \preceq t \Longleftrightarrow s_{i} \leq t_{i}, 1 \leq i \leq N$. We can therefore assume that the sample space $\Omega$ is the space of rcll functions from $\mathbb{R}^{N}$ into $\mathbb{R}^{d}$. Let $\lambda_{d}$ be $d$-dimensional Lebesgue measure. Fix a Borel set $G \subset \mathbb{R}_{+}^{N}$. Let $F \subset \mathbb{R}^{d}$ be an analytic set. $F$ is called essentially polar provided $P\{X(G) \cap(F-y) \neq \emptyset\}=P^{y}\{X(G) \cap F \neq \emptyset\}=0$ for $\lambda_{d}$-a.e. $y$, while $F$ is said to be polar if $P^{y}\{X(G) \cap F \neq \emptyset\}=0$ for every $y$. Let $P^{x}, x \in \mathbb{R}^{d}$, be the probability measure on $(\Omega, \mathcal{F})$ given by the path spatial-shift $P^{x}\{\bullet\}=P^{0}\{x+\bullet\}$, where $x+A=\{x+\omega: \omega \in A\}, A \in \mathcal{F}, P^{0}=P$, and $x+\omega \in \Omega$ is defined by $(x+\omega)(t)=x+\omega(t), t \in \mathbb{R}^{N}$. Hence, we have a $\sigma$-finite measure $P_{\lambda}$ on $(\Omega, \mathcal{F})$ given by $P_{\lambda}(\bullet)=\int_{\mathbb{R}^{d}} P^{x}(\bullet) d x$. (In [4], the superscript notation $P^{\nu}$ is used, where $P^{\nu}(\bullet)=\int_{\mathbb{R}^{d}} P^{x}(\bullet) \nu(d x)$ and $\nu$ is a measure on $\mathbb{R}^{d}$.) Assume that $X$ is in the canonical form. It is easy to see that

$$
P_{\lambda}\left\{X_{t} \in F \text { for some } t \in G\right\}=E\left\{\lambda_{d}(X(G)-F)\right\},
$$

where $E_{1}-E_{2}=\left\{x-y: x \in E_{1}, y \in E_{2}\right\}$ for nonempty sets $E_{1}$ and $E_{2}$ of $\mathbb{R}^{d}$. By Fubini's theorem, $E\left\{\lambda_{d}(X(G)-F)\right\}=\int_{\mathbb{R}^{d}} P\{X(G) \cap(F-y) \neq \emptyset\} d y$. Thus, $F$ is essentially polar iff $E\left\{\lambda_{d}(X(G)-F)\right\}=0$ iff $P_{\lambda}\left\{X_{t} \in F\right.$ for some $\left.t \in G\right\}=0$. We will call the Choquet capacity (with respect to the class of compact sets, or of open sets):

$$
\Gamma(F)=P_{\lambda}\left\{X_{t} \in F \text { for some } t \in G\right\}
$$

the $Y$-capacity. ( $Y$ stands for one of the author's initials. We are trying to make the notation for a new terminology as handy as possible.) Immediately, $F$ is essentially polar iff $\Gamma(F)=0$. It will soon become clear that $\Gamma$ is a natural generalization of the $q$-capacity $(q>0)$ for Markov processes, the $q$-capacity for Lévy processes, and

Received by the editors February 13, 2012 and, in revised form, October 13, 2012 and November 10,2012 .

2010 Mathematics Subject Classification. Primary 60J45, 60G60; Secondary 60G17.

Key words and phrases. Random fields, capacity, energy, potential theory, Markov fields. 
the "classical capacity" of [4 for a class of Markov fields. Define

$$
Q_{\mu}^{X}(\xi)=\int_{\mathbb{R}^{N}} \int_{\mathbb{R}^{N}} E e^{i \xi \cdot\left(X_{s}-X_{t}\right)} \mu(d s) \mu(d t),
$$

where $\mu$ is a probability measure in $\mathbb{R}^{N}$ and $\xi \in \mathbb{R}^{d}$. Let $\mathcal{P}(B)$ denote the set of probability measures $\mu$ on $B$, i.e., $\mu\left(B^{c}\right)=0$. We will call

$$
\mathcal{E}(F)=\inf \left\{(2 \pi)^{-d} \int_{\mathbb{R}^{d}}|\hat{\mu}(\xi)|^{2} Q_{\eta}^{X}(\xi) d \xi: \mu \in \mathcal{P}(F), \eta \in \mathcal{P}(G)\right\}
$$

the $Y$-energy of $F$, where $\hat{\mu}$ is the Fourier transform of $\mu$. $Y$-energy is clearly an analog of the Hawkes energy for Lévy processes. See below for the Hawkes energy. (By standard norm argument, for any compact $F$, if $F^{o} \neq \emptyset$ and $\mathcal{E}(F)<\infty$, then

$$
\mathcal{E}(F)=\inf \left\{(2 \pi)^{-d} \int_{\mathbb{R}^{d}}\left|\widehat{\lambda_{F}}(\xi)\right|^{2} Q_{\eta}^{X}(\xi) d \xi: \eta \in \mathcal{P}(G)\right\},
$$

where $\lambda_{F}$ is the normalized Lebesgue measure on $F$.) The subject matter of potential theory, among other things, is to understand how energy is related to capacity. One always has

$$
[\Gamma(F)]^{-1} \leq \mathcal{E}(F)
$$

and under a rather general regularity assumption on $X$

$$
\text { either } \mathcal{E}(F) / 2 \leq[\Gamma(F)]^{-1} \text { or } \Gamma(F)>0 \Longrightarrow \mathcal{E}(F)<\infty \text {. }
$$

In most applications, one is concerned about whether capacity is zero or positive (or energy is finite or infinite), and not about the actual value of either $\Gamma(F)$ or $\mathcal{E}(F)$.

(1.5) is not new for Markov processes and for the Markov fields of 4. For a simple list of standard original references about the subject, we send a new reader of the present paper to [1, Chap. VI], [5], and [4]. The following results hold for a general state space $S$ and for any reference measure $\xi$ on $S$. Since the present paper is about fields in $\mathbb{R}^{d}$, where $P_{\lambda}$ is with respect to $\lambda_{d}$, we will fix the state space $\mathbb{R}^{d}$ and the reference measure $\lambda_{d}$.

Let $M$ be a Markov process taking values in $\mathbb{R}^{d}$ in duality relative to $\lambda_{d}$ with the canonical $q$-potential density $u^{q}(x, y)$ for each $q>0$. There exists a unique Radon measure $\mu_{F}^{q}$ (the $q$-capacitary measure of $F$ ) with $\operatorname{supp}\left(\mu_{F}^{q}\right) \subset \bar{F}$ such that

$$
P^{x}\left(M\left(\left(0, \tau^{q}\right)\right) \cap F \neq \emptyset\right)=\int_{\bar{F}} u^{q}(x, y) \mu_{F}^{q}(d y), \forall x,
$$

where $\tau^{q}$ is an exponential time at rate $q$ independent of $M$. The Choquet capacity $\mathcal{C}^{q}(F)=\mu_{F}^{q}(\bar{F})$ is called the $q$-capacity of $F$. Furthermore, $\mathcal{C}^{q}(F)=\sup \{\mu(F)$ : $\int_{F} u^{q}(x, y) \mu(d y) \leq 1$ for all $\left.x, \operatorname{supp}(\mu) \subset F\right\}$ and $\mathcal{C}^{q}(F)=0$ for some $q>0$ (hence all $q>0)$ iff $F$ is polar with $G=(0, \infty)$. Note that for $M$, polar = essentially polar (cf. the notion of $\phi$-potential density in Section 3). We have

$$
\begin{aligned}
& \Gamma^{q}(F)=P_{\lambda}\left(M\left(\left(0, \tau^{q}\right)\right) \cap F \neq \emptyset\right)=\int_{\mathbb{R}^{d}} P^{x}\left(M\left(\left(0, \tau^{q}\right)\right) \cap F \neq \emptyset\right) d x \\
& =\int_{\mathbb{R}^{d}} \int_{\bar{F}} u^{q}(x, y) \mu_{F}^{q}(d y) d x=\int_{\bar{F}} \int_{\mathbb{R}^{d}} u^{q}(x, y) d x \mu_{F}^{q}(d y) \\
& =\int_{\bar{F}} q^{-1} \mu_{F}^{q}(d y)=q^{-1} \mu_{F}^{q}(\bar{F})=q^{-1} \mathcal{C}^{q}(F) .
\end{aligned}
$$


If in addition $M$ is symmetric, i.e., $u^{q}(x, y)$ is a symmetric kernel, then $M$ has a transition density and one defines the $q$-energy $\mathcal{E}^{q}(F)$ of $F$ by $\mathcal{E}^{q}(F)=$ $\inf \left\{\iint u^{q}(x, y) \mu(d x) \mu(d y): \mu \in \mathcal{P}(F)\right\}$. One always has $\mathcal{C}^{q}(F)=0 \Longleftrightarrow \mathcal{E}^{q}(F)=$ $\infty, \mathcal{C}^{q}(F)=\infty \Longleftrightarrow \mathcal{E}^{q}(F)=0$, and if $\mathcal{C}^{q}(F) \in(0, \infty)$, then $\left[\mathcal{C}^{q}(F)\right]^{-1}=\mathcal{E}^{q}(F)=$ $\iint u^{q}(x, y) \mu_{F}^{q, *}(d x) \mu_{F}^{q, *}(d y)$, or equivalently $\mathcal{C}^{q}(F)=\iint u^{q}(x, y) \mu_{F}^{q}(d x) \mu_{F}^{q}(d y)$, where $\mu_{F}^{q, *}=\left[\mathcal{C}^{q}(F)\right]^{-1} \mu_{F}^{q}$.

Remark. As $q$ decreases to $0, u^{q}(x, y)$ increases to a function $u(x, y)$. If $X$ is transient, $u$ is the canonical duality density (a.e. finite) of $(U, \widehat{U})$. In the transient case, $\mu_{F}^{q}$ converges weakly to a Radon measure $\mu_{F}$ with $\operatorname{supp}\left(\mu_{F}\right) \subset \bar{F}$ as $q \downarrow 0$ such that $P^{x}(M((0, \infty)) \cap F \neq \emptyset)=\int_{\bar{F}} u(x, y) \mu_{F}(d y), \forall x . \mathcal{C}(F)=\mu_{F}(\bar{F})$ is called the capacity of $F$. Our $Y$-capacity $\Gamma(F)$ with $G=(0, \infty)$ is not necessarily a multiple of $\mathcal{C}(F)$, since $P_{\lambda}(M((0, \infty)) \cap F \neq \emptyset)=0$ or $\infty$ always. On the other hand, for a transient Hunt process $M, \mu_{F}$ can be defined without assuming that $M$ has a dual. See [2, Chap. 5]. Hence for a Hunt process $M$, as long as $U^{q}(x, \cdot)$ has a density $u^{q}(x, \cdot)$ for each $x, \mu_{F}^{q}$ can be defined via killing $M$ at $\tau^{q}$.

Let $Z$ be an arbitrary Lévy process in $\mathbb{R}^{d}$. By Hunt's switching lemma, there is a unique Radon measure $\mu_{F}^{q}$ for each $q>0$ (the $q$-capacitary measure of $F$ ) with $\operatorname{supp}\left(\mu_{F}^{q}\right) \subset \bar{F}$ such that $\mu_{F}^{q} \widehat{U}^{q}(d x)=E^{x}\left(e^{-q T_{F}}\right) d x$, where $\widehat{U}^{q}$ is the $q$-resolvent kernel of the dual $\widehat{Z}=-Z$ and $T_{F}=\inf \left\{t>0: Z_{t} \in F\right\} \cdot \mathcal{C}^{q}(F)=\mu_{F}^{q}(\bar{F})$ is called the $q$-capacity of $F$. If $Z$ has a strong Feller resolvent, then $\mu_{F}^{q}$ agrees with the one for $M$. It is important to know that $\mathcal{C}^{q}(F)=0$ for some $q>0$ (hence for all $q>0)$ iff $F$ is essentially polar with $G=(0, \infty)$. The Hawkes energy for Lévy processes is defined by $\mathcal{E}^{q}(F)=\inf \left\{(2 \pi)^{-d} \int_{\mathbb{R}^{d}}|\hat{\mu}(\xi)|^{2} Q_{\kappa^{q}}^{Z}(\xi) d \xi: \mu \in \mathcal{P}(F)\right\}$, where $\kappa^{q}(d t)=q e^{-q t} d t, t \geq 0$, for each $q>0$. If $Z$ is symmetric and has a strong Feller resolvent, then $\mathcal{E}^{q}(F)$ coincides with the one for $M$. For an arbitrary Lévy process $Z$ in any event, one always has $\mathcal{E}^{q}(F) \leq\left[\mathcal{C}^{q}(F)\right]^{-1} \leq 4 \mathcal{E}^{q}(F)$.

Let $X_{t}=\left(X_{t_{1}}^{1}, \cdots, X_{t_{N}}^{N}\right), t=\left(t_{1}, \cdots, t_{N}\right) \in \mathbb{R}_{+}^{N}$, where $X^{i}: \mathbb{R}_{+} \rightarrow \mathbb{R}^{d_{i}}$ are independent Markov processes. Assume that $X^{i}$ is in duality relative to $\lambda_{d_{i}}$. Let $u^{i, q_{i}}$ be the canonical $q_{i}$-potential density of $X^{i}, q_{i}>0 . X$ is a Markov field taking values in $\mathbb{R}^{d}$, where $d=d_{1}+\cdots+d_{N}$. (See Section 3.) Let $\tau_{1}^{q_{1}}, \cdots, \tau_{N}^{q_{N}}$ be independent exponential times also independent of $X^{1}, \cdots, X^{N}$, where $\tau_{i}^{q_{i}}$ is at rate $q_{i}$, let $\left(0, \tau^{\mathbf{q}}\right)=\left(0, \tau_{1}^{q_{1}}\right) \times \cdots \times\left(0, \tau_{N}^{q_{N}}\right)$, let $q=q_{1} \cdots q_{N}$, and let $u^{\mathbf{s}, \mathbf{q}}(x, y)$ be the complete symmetrization of the product $u^{1, q_{1}}\left(x^{1}, y^{1}\right) \cdots u^{N, q_{N}}\left(x^{N}, y^{N}\right)$, where $x=\left(x^{1}, \cdots, x^{N}\right), y=\left(y^{1}, \cdots, y^{N}\right), x^{i}, y^{i} \in \mathbb{R}^{d_{i}}$. As in [4], let $Q=P_{\lambda}$ with respect to $\lambda_{d}, \mathcal{E}^{q}(F)=\inf \left\{\iint u^{\mathbf{s}, \mathbf{q}}(x, y) \mu(d x) \mu(d y): \mu \in \mathcal{P}(F)\right\}$, the "classical energy" of [4], and $\Gamma^{q}(F)=q P_{\lambda}\left\{X_{t} \in F\right.$ for some $\left.t \in\left(0, \tau^{\mathbf{q}}\right)\right\}$, the "classical capacity" of [4. One always has $2^{-3 N} \mathcal{E}^{q}(F) \leq\left[\Gamma^{q}(F)\right]^{-1}$. If each $X^{i}$ is symmetric, then $\left[\Gamma^{q}(F)\right]^{-1} \leq 2^{N-1} \mathcal{E}^{q}(F)$. Applying the potential-theoretic result to hitting probability, one always has $P^{x}\left\{X_{t} \in F\right.$ for some $\left.t \in(0, \infty)^{N}\right\}=0$ for every $x$ if $\mathcal{E}^{q}(F)=\infty$ for some $q>0$ (hence for all $q>0$ ). For fixed $x=\left(x^{1}, \cdots, x^{N}\right), P^{x}\left\{X_{t} \in F\right.$ for some $\left.t \in(0, \infty)^{N}\right\}>0$ if for each $i, X^{i}$ is symmetric, $u^{i, q_{i}}\left(x^{i}, y^{i}\right)>0$ for a.e. $y^{i}$, and $\mathcal{E}^{q}(F)<\infty$.

While an infinite number of probability measures $\eta$ are associated with $G$ if $G$ is not a finite set, the $Y$-energy $\mathcal{E}(F)$ in (1.4) is far more useful in computation and in making a comparison between two fields than the "additive functional energy" $I_{\mathrm{HRM}}$ of [4]. On the other hand, for a general field, even for a Markov field of [4], it is not always possible to obtain a closed-form energy kernel. (The symmetrized 
energy kernel $u^{\mathbf{s}, \mathbf{q}}$ of [4] works essentially for a subclass of the fields of [4.) However one can show that for a large number of types of fields, e.g., symmetric Lévy sheets, anisotropic Gaussian fields, fields with stationary increments, just to name a few, the closed-form 1-kernel $Q_{\kappa}^{Y}(\xi)$, where $Y: \mathbb{R}^{N} \rightarrow \mathbb{R}^{d}$ is some field with stationary increments and $\kappa(d t)=e^{-\sum_{j=1}^{N} t_{j}} d t, t=\left(t_{1}, \cdots, t_{N}\right) \in \mathbb{R}_{+}^{N}$, vanishing elsewhere, is a correct energy kernel. While a basic function of potential theory is to determine hitting probabilities (see Section 3 in the case of Markov fields), there are several complete non-potential-theoretic results on hitting probabilities in relatively elementary situations. Generally speaking, potential theory is inspired by many of these non-potential-theoretic ideas. At last, harmonic analysis has replaced classical Gaussian-type density estimates. [6] and [3] do not use potential theory for example, but [6] showed that for $(N, d)$ Brownian sheets, hitting probability is comparable to Riesz capacity of order $d-2 N$ and [3] obtained the same result, when $N=2$, for the non-Gaussian continuous solution to a system of $d$ nonlinear hyperbolic spds in two variables.

In Section 2 we prove (1.5). In Section 3 we obtain hitting probability results for Markov fields from the potential theory developed in the paper. Finally, in Section 4 we establish the links from fractional fields to potential theory.

\section{THE b-CONDITION}

The gist of proving many interesting potential-theoretic results lies in a new potential-theoretic concept, called the b-condition. Let $X: \mathbb{R}^{N} \rightarrow \mathbb{R}^{d}$ be an rcll field in the usual partial order. Assume that the sample space $\Omega$ of $X$ is the space of rcll functions from $\mathbb{R}^{N}$ into $\mathbb{R}^{d}$. Fix a sufficiently large $l \in(0, \infty)$. Consider a measurable mapping $\mathbf{b}: \Omega \rightarrow \Omega_{+}$, where $\Omega_{+}$is the space of rcll functions from $\mathbb{R}_{+}^{N}$ into $\mathbb{R}^{d}$. So, $\mathbf{b}$ is a random variable taking values in $\Omega_{+}$. Let $\Omega_{l}$ be the space of rcll functions from $[0, l)^{N}$ into $\mathbb{R}^{d}$, which is the sample space of the field $X_{t}^{l}=X_{t}, t \in$ $[0, l)^{N}$. Let $r: \Omega \rightarrow \Omega_{l}$ be the restriction mapping, i.e., $r(\omega)(t)=\omega(t), t \in[0, l)^{N}$, for $\omega \in \Omega$. Assume that $\mathbf{b}$ has the properties: (1) $\mathbf{b}$ is independent of $X^{l}$. (2) There exists an event $\Omega^{\prime} \subset \Omega$ with $r\left(\Omega^{\prime}\right)=\Omega_{l}$ such that $r\left[\mathbf{b}\left(\omega^{\prime}\right)\right]=r\left(\omega^{\prime}\right)$ for each $\omega^{\prime} \in \Omega^{\prime}$. (Here the second restriction makes sense because $[0, l)^{N} \subset \mathbb{R}_{+}^{N}$.) If $\mathbf{b}$ exists for some arbitrarily large $l$, we will simply say that $X$ has $\mathbf{b}$, or just the $\mathbf{b}$-condition holds. ("b-condition" is not a cool name. One may rather call it the "path rigid independence" condition.) We can also define $\mathbf{b}$ in the same way as above yet by replacing $\Omega_{+}$by $\Omega$ and by changing the domain $[0, l)^{N}$ of $\Omega_{l}$ into $\mathbb{R}^{m_{1}} \times[-l, l) \times \mathbb{R}^{m_{2}}$, where $m_{1}+m_{2}=N-1$, or $\mathbb{R}^{m_{1}} \times[-l, l)^{n} \times \mathbb{R}^{m_{2}}$ with $m_{1}+m_{2}+n=N$, or $[0, l)^{n} \times \mathbb{R}^{m}$ with $n+m=N$, depending on the field $X$ under consideration and the need.

Note that $\mathbf{b}$ only has to be defined on an a.s. event $A$ of $\Omega$. Define $\mathbf{b}_{A}: A \rightarrow \Omega_{+}$ $\left(\mathbf{b}_{A}: A \rightarrow \Omega\right)$ such that (1) $\mathbf{b}_{A}$ is independent of $X^{l}$. (2) For some event $A^{\prime} \subset A$ with $r\left(A^{\prime}\right)=r(A), r\left[\mathbf{b}\left(\omega^{\prime}\right)\right]=r\left(\omega^{\prime}\right)$ for each $\omega^{\prime} \in A^{\prime}$. Define $\mathbf{b}(\omega)=\mathbf{b}_{A}(\omega)$ for $\omega \in A$ and $\mathbf{b}(\omega)=\omega_{+}(\mathbf{b}(\omega)=\omega)$ for $\omega \in \Omega \backslash A$, where $\omega_{+}(t)=\omega(t), t \in \mathbb{R}_{+}^{N}$. Clearly, $\mathbf{b}$ is independent of $X^{l}$. Extend $\Omega_{l} \backslash r(A)$ to an event $B$ of $\Omega$ in an arbitrary way. Set $\Omega^{\prime}=A^{\prime} \cup B$. Clearly, $r\left(\Omega^{\prime}\right)=\Omega_{l}$. Note that $A \cap B=\emptyset$ since $r(B)=\Omega_{l} \backslash r(A)$. For each $\omega^{\prime} \in B, \mathbf{b}\left(\omega^{\prime}\right)=\omega_{+}^{\prime}\left(\mathbf{b}\left(\omega^{\prime}\right)=\omega^{\prime}\right)$. Thus, $r\left(\mathbf{b}\left(\omega^{\prime}\right)\right)=r\left(\omega_{+}^{\prime}\right)=r\left(\omega^{\prime}\right)$ $\left(r\left(\mathbf{b}\left(\omega^{\prime}\right)\right)=r\left(\omega^{\prime}\right)\right)$. It follows that $r\left[\mathbf{b}\left(\omega^{\prime}\right)\right]=r\left(\omega^{\prime}\right)$ for each $\omega^{\prime} \in \Omega^{\prime}$.

Let $U, W: \mathbb{R}^{N} \rightarrow \mathbb{R}^{d}$ be two rcll fields on the same sample space $\Omega$, the space of rcll functions from $\mathbb{R}^{N}$ into $\mathbb{R}^{d}$. We say that $U$ is adapted to $W$ if $U_{t}, t \in$ $[0, l]^{N}\left(U_{t}, t \in[-l, l] \times \mathbb{R}^{N-1}\right)$ are measurable to the $\sigma$-field $\sigma\left(W_{t}: t \in[0, l]^{N}\right)$ 
$\left(\sigma\left(W_{t}: t \in[-l, l] \times \mathbb{R}^{N-1}\right)\right)$ for all large $l \in(0, \infty)$. Note that in some cases, $U_{t}, t \in$ $\mathbb{R}^{N}$, are measurable to $\sigma\left(W_{t}: t \in\left[-l_{1}, l_{1}\right] \times \mathbb{R}^{N-1}\right)$ for some sufficiently large $l_{1}$. It is elementary that if $W$ has $\mathbf{b}$, so does $U$. This assertion can be proved as follows. Since $U$ is adapted to $W, \mathbf{b}$ is independent of $U^{l}$ as well. Thus, $U$ has $\mathbf{b}$, which is just the same $\mathbf{b}$ for $W$.

Lemma 2.1. Let $X^{i}: \mathbb{R}^{N_{i}} \rightarrow \mathbb{R}^{d_{i}}$ be an rcll field having $\mathbf{b}_{i}, 1 \leq i \leq n$. Assume that $X^{i}$ are independent. Then the fields $Z_{t}^{1}=\left(X_{t}^{1}, \cdots, X_{t}^{n}\right), Z_{t}^{2}=\left(X_{t_{1}}^{1}, \cdots, X_{t_{n}}^{n}\right)$, $t=\left(t_{1}, \cdots, t_{n}\right), Z_{t}^{3}=X_{t}^{1}+\cdots+X_{t}^{n}$, and $Z_{t}^{4}=X_{t_{1}}^{1}+\cdots+X_{t_{n}}^{n}, t=\left(t_{1}, \cdots, t_{n}\right)$, all have $\mathbf{b}$, where $\left(Z^{2}\right)^{l}$ and $\left(Z^{4}\right)^{l}$ have domain $\left([-l, l) \times \mathbb{R}^{N_{1}-1}\right) \times \cdots \times\left([-l, l) \times \mathbb{R}^{N_{n}-1}\right)$. Here we have assumed that every $\left(X^{i}\right)^{l}$ has domain $[-l, l) \times \mathbb{R}^{N_{i}-1}$ and $\mathbf{b}_{i}: \Omega_{i} \rightarrow \Omega_{i}$ for the sake of simplicity. But clearly, the arguments are the same for any kind of combination of the domains and for $\mathbf{b}_{i}: \Omega_{i} \rightarrow\left(\Omega_{i}\right)_{+}$. For instance, if $d_{1}=d_{2}=d$, $X^{1}: \mathbf{b}_{1}: \Omega_{1} \rightarrow\left(\Omega_{1}\right)_{+},[0, l)^{N_{1}}, X^{2}: \mathbf{b}_{2}: \Omega_{2} \rightarrow \Omega_{2},[-l, l)^{N_{2}}$, then $Z_{t}^{4}=X_{t_{1}}^{1}+X_{t_{2}}^{2}:$ $[0, l)^{N_{1}} \times[-l, l)^{N_{2}}, \mathbf{b}: \Omega_{Z} \rightarrow \Omega^{*}$, where $\Omega^{*}$ is the space of rcll functions from $\mathbb{R}_{+}^{N_{1}} \times \mathbb{R}^{N_{2}}$ into $\mathbb{R}^{d}$.

Conversely, if $Z_{t}^{5}=\left(X_{t}^{1}, X_{t}^{2}\right)$ has $\mathbf{b}$, then both $X^{1}$ and $X^{2}$ have $\mathbf{b}$, where $X^{1}$ and $X^{2}$ are not necessarily independent.

Proof. $Z^{1}$ : Let $\theta$ be an rcll function from $\mathbb{R}^{N}$ into $\mathbb{R}^{d_{1}+\cdots+d_{n}}$. Then $\theta(t)=\left(\omega_{1}(t)\right.$, $\left.\cdots, \omega_{n}(t)\right)$ with $\omega_{i} \in \Omega_{i}$, where the $\omega_{i}$ are unique. Thus, $\mathbf{b}(\theta)(t)=\left(\mathbf{b}_{1}\left(\omega_{1}\right)(t)\right.$, $\left.\cdots, \mathbf{b}_{n}\left(\omega_{n}\right)(t)\right)$ is well defined. Since $\mathbf{b}_{i}$ are independent, $\mathbf{b}$ is independent of $\left(Z^{1}\right)^{l}$. Let $\bar{\Omega}^{\prime}=\left\{\left(\omega_{1}, \cdots, \omega_{n}\right): \omega_{i} \in \Omega_{i}^{\prime}\right\}$. We see that $r\left(\bar{\Omega}^{\prime}\right)=\bar{\Omega}_{l}$, where $\bar{\Omega}_{l}$ is the space of rcll functions from $[-l, l) \times \mathbb{R}^{N-1}$ into $\mathbb{R}^{d_{1}+\cdots+d_{n}}$. For each $\theta=\left(\omega_{1}, \cdots, \omega_{n}\right), \omega_{i} \in$ $\Omega_{i}^{\prime}$, we have $r(\mathbf{b}(\theta))=\left(r_{1}\left(\mathbf{b}_{1}\left(\omega_{1}\right)\right), \cdots, r_{n}\left(\mathbf{b}_{n}\left(\omega_{n}\right)\right)\right)=\left(r_{1}\left(\omega_{1}\right), \cdots, r_{n}\left(\omega_{n}\right)\right)=r(\theta)$.

$Z^{2}$ : In this case, $\left(Z^{2}\right)^{l}$ has domain $\left([-l, l) \times \mathbb{R}^{N_{1}-1}\right) \times \cdots \times\left([-l, l) \times \mathbb{R}^{N_{n}-1}\right)$. Let $A=\left\{\left(\omega_{1}, \cdots, \omega_{n}\right): \omega_{i} \in \Omega_{i}\right\}$. Then $P(A)=1$. Note that $\left(\omega_{1}, \cdots, \omega_{n}\right)=$ $\left(\omega_{1}^{\prime}, \cdots, \omega_{n}^{\prime}\right) \Longrightarrow \omega_{i}=\omega_{i}^{\prime}$. Thus, for $\theta=\left(\omega_{1}, \cdots, \omega_{n}\right) \in A, \mathbf{b}(\theta)=\left(\mathbf{b}_{1}\left(\omega_{1}\right)\right.$, $\left.\cdots, \mathbf{b}_{n}\left(\omega_{n}\right)\right)$ is well defined. Since $\mathbf{b}_{i}$ are independent, $\mathbf{b}$ is independent of $\left(Z^{2}\right)^{l}$. Let $A^{\prime}=\left\{\left(\omega_{1}, \cdots, \omega_{n}\right): \omega_{i} \in \Omega_{i}^{\prime}\right\}$. Then $A^{\prime} \subset A$ and $r\left(A^{\prime}\right)=r(A)$ since $r_{i}\left(\Omega_{i}^{\prime}\right)=\left(\Omega_{i}\right)_{l}$. For each $\theta \in A^{\prime}, t_{i} \in[-l, l) \times \mathbb{R}^{N_{i}-1}$, we have $\left(\mathbf{b}_{1}\left(\omega_{1}\right)\left(t_{1}\right), \cdots, \mathbf{b}_{n}\left(\omega_{n}\right)\left(t_{n}\right)\right)=$ $\left(\omega_{1}\left(t_{1}\right), \cdots, \omega_{n}\left(t_{n}\right)\right)$, i.e., $r(\mathbf{b}(\theta))=r(\theta)$.

$Z^{3}$ : Let $A=\left\{\omega_{1}(t)+\cdots+\omega_{n}(t): \omega_{i} \in \Omega_{i}\right\}$. Then $P(A)=1$. The relation $\omega_{1}(t)+$ $\cdots+\omega_{n}(t)=\omega_{1}^{\prime}(t)+\cdots+\omega_{n}^{\prime}(t), \forall t, \omega_{i}, \omega_{i}^{\prime} \in \Omega_{i}$, divides $A$ into disjoint equivalence classes. Denote a class by $C$. Then there exists a well-defined measurable map b : $A \rightarrow \Omega$ such that there is an $\omega_{1}(t)+\cdots+\omega_{n}(t) \in C$ for each class $C$ and for any $\theta^{\prime}(t)=\omega_{1}^{\prime}(t)+\cdots+\omega_{n}^{\prime}(t) \in C, \mathbf{b}\left(\theta^{\prime}\right)(t)=\mathbf{b}_{1}\left(\omega_{1}\right)(t)+\cdots+\mathbf{b}_{n}\left(\omega_{n}\right)(t)$, and if $\omega_{i}^{\prime} \in \Omega_{i}^{\prime}$ for all $i$, then $\omega_{i} \in \Omega_{i}^{\prime}$ for all $i$. Since $\mathbf{b}_{i}$ are independent, $\mathbf{b}$ is independent of $\left(Z^{3}\right)^{l}$. Let $A^{\prime}=\left\{\omega_{1}(t)+\cdots+\omega_{n}(t): \omega_{i} \in \Omega_{i}^{\prime}\right\}$. Clearly, $A^{\prime} \subset A$. Also $r\left(A^{\prime}\right)=r(A)$ because $r_{i}\left(\Omega_{i}^{\prime}\right)=\left(\Omega_{i}\right)_{l}$. For each $\theta^{\prime} \in A^{\prime}, t \in[-l, l) \times \mathbb{R}^{N}$, assuming $\theta^{\prime} \in C$, we have $\mathbf{b}\left(\theta^{\prime}\right)(t)=\mathbf{b}_{1}\left(\omega_{1}\right)(t)+\cdots+\mathbf{b}_{n}\left(\omega_{n}\right)(t)=\omega_{1}(t)+\cdots+\omega_{n}(t)=\omega_{1}^{\prime}(t)+\cdots+\omega_{n}^{\prime}(t)$, i.e., $r\left(\mathbf{b}\left(\theta^{\prime}\right)\right)=r\left(\theta^{\prime}\right)$.

$Z^{4}$ : In this case, $\left(Z^{4}\right)^{l}$ has domain $\left([-l, l) \times \mathbb{R}^{N_{1}-1}\right) \times \cdots \times\left([-l, l) \times \mathbb{R}^{N_{n}-1}\right)$. First assume that $X_{0}^{i}=0$ a.s. Let $A=\left\{\omega_{1}\left(t_{1}\right)+\cdots+\omega_{n}\left(t_{n}\right): \omega_{i} \in \Omega_{i}, \omega_{i}(0)=0\right\}$. Then $P(A)=1$. If $\omega_{1}\left(t_{1}\right)+\cdots+\omega_{n}\left(t_{n}\right)=\omega_{1}^{\prime}\left(t_{1}\right)+\cdots+\omega_{n}^{\prime}\left(t_{n}\right), \forall t_{i}$, where $\omega_{i}, \omega_{i}^{\prime} \in \Omega_{i}$, $\omega_{i}(0)=\omega_{i}^{\prime}(0)=0$, then taking $t_{j}=0$ for all $j \neq i$ shows that $\omega_{i}\left(t_{i}\right)=\omega_{i}^{\prime}\left(t_{i}\right), \forall t_{i}$, i.e., $\omega_{i}=\omega_{i}^{\prime}$. Therefore, for $\theta(t)=\omega_{1}\left(t_{1}\right)+\cdots+\omega_{n}\left(t_{n}\right) \in A, \mathbf{b}(\theta)(t)=\mathbf{b}_{1}\left(\omega_{1}\right)\left(t_{1}\right)+$ $\cdots+\mathbf{b}_{n}\left(\omega_{n}\right)\left(t_{n}\right)$ is well defined. Since $X_{0}^{i}=0$ a.s. and since $\mathbf{b}_{i}$ are independent, $\mathbf{b}$ is independent of $\left(Z^{4}\right)^{l}$. Let $A^{\prime}=\left\{\omega_{1}\left(t_{1}\right)+\cdots+\omega_{n}\left(t_{n}\right): \omega_{i} \in \Omega_{i}^{\prime}, \omega_{i}(0)=0\right\}$. 
We see that $A^{\prime} \subset A$ and $r\left(A^{\prime}\right)=r(A)$ because $r_{i}\left(\Omega_{i}^{\prime}\right)=\left(\Omega_{i}\right)_{l}$. For each $\theta \in A^{\prime}$ and $t_{i} \in[-l, l) \times \mathbb{R}^{N_{i}-1}$, we have $\mathbf{b}_{1}\left(\omega_{1}\right)\left(t_{1}\right)+\cdots+\mathbf{b}_{n}\left(\omega_{n}\right)\left(t_{n}\right)=\omega_{1}\left(t_{1}\right)+\cdots+\omega_{n}\left(t_{n}\right)$, i.e., $r(\mathbf{b}(\theta))=r(\theta)$.

We can now remove the assumption that $X_{0}^{i}=0$ a.s. Let $A=\left\{\omega_{1}\left(t_{1}\right)+\right.$ $\left.\cdots+\omega_{n}\left(t_{n}\right): \omega_{i} \in \Omega_{i}\right\}$. Then $P(A)=1$. The relation $\omega_{1}\left(t_{1}\right)+\cdots+\omega_{n}\left(t_{n}\right)=$ $\omega_{1}^{\prime}\left(t_{1}\right)+\cdots+\omega_{n}^{\prime}\left(t_{n}\right), \forall t_{i}, \omega_{i}, \omega_{i}^{\prime} \in \Omega_{i}$, divides $A$ into disjoint equivalence classes. Denote a class by $C$. Then there exists a well-defined measurable map $\mathbf{b}: A \rightarrow \Omega$ such that there is an $\omega_{1}\left(t_{1}\right)+\cdots+\omega_{n}\left(t_{n}\right) \in C$ for each class $C$ and for any $\theta^{\prime}(t)=\omega_{1}^{\prime}\left(t_{1}\right)+\cdots+\omega_{n}^{\prime}\left(t_{n}\right) \in C, \mathbf{b}\left(\theta^{\prime}\right)(t)=\mathbf{b}_{1}\left(\omega_{1}\right)\left(t_{1}\right)+\cdots+\mathbf{b}_{n}\left(\omega_{n}\right)\left(t_{n}\right)$, and if $\omega_{i}^{\prime} \in \Omega_{i}^{\prime}$ for all $i$, then $\omega_{i} \in \Omega_{i}^{\prime}$ for all $i$. Since $\mathbf{b}_{i}$ are independent, $\mathbf{b}$ is independent of $\left(Z^{4}\right)^{l}$. Let $A^{\prime}=\left\{\omega_{1}\left(t_{1}\right)+\cdots+\omega_{n}\left(t_{n}\right): \omega_{i} \in \Omega_{i}^{\prime}\right\}$. Clearly, $A^{\prime} \subset A$. Also $r\left(A^{\prime}\right)=r(A)$ because $r_{i}\left(\Omega_{i}^{\prime}\right)=\left(\Omega_{i}\right)_{l}$. For each $\theta^{\prime} \in A^{\prime}, t_{i} \in[-l, l) \times \mathbb{R}^{N_{i}-1}$, assuming $\theta^{\prime} \in C$, we have $\mathbf{b}\left(\theta^{\prime}\right)(t)=\mathbf{b}_{1}\left(\omega_{1}\right)\left(t_{1}\right)+\cdots+\mathbf{b}_{n}\left(\omega_{n}\right)\left(t_{n}\right)=\omega_{1}\left(t_{1}\right)+\cdots+\omega_{n}\left(t_{n}\right)=$ $\omega_{1}^{\prime}\left(t_{1}\right)+\cdots+\omega_{n}^{\prime}\left(t_{n}\right)$, i.e., $r\left(\mathbf{b}\left(\theta^{\prime}\right)\right)=r\left(\theta^{\prime}\right)$.

The last assertion of the lemma can be proved as follows. Let $\pi$ be the first component projection, i.e., $\pi\left(\omega_{1}, \omega_{2}\right)=\omega_{1}$, where $\omega_{1} \in \Omega_{1}$. Let $\mathbf{b}$ and $\Omega^{\prime}$ be for $Z^{5}$. We choose a measurable extention from $\Omega_{1}$ to $\Omega$ : $\omega_{1} \mapsto\left(\omega_{1}, e\left(\omega_{1}\right)\right) \in \Omega$ such that for each $\omega_{1} \in \pi\left(\Omega^{\prime}\right),\left(\omega_{1}, e\left(\omega_{1}\right)\right) \in \Omega^{\prime}$. Clearly, $r\left(\pi\left(\Omega^{\prime}\right)\right)=\left(\Omega_{1}\right)_{l}$. So let $\Omega_{1}^{\prime}=\pi\left(\Omega^{\prime}\right)$. Now define $\mathbf{b}_{1}\left(\omega_{1}\right)=\pi\left[\mathbf{b}\left(\left(\omega_{1}, e\left(\omega_{1}\right)\right)\right)\right], \omega_{1} \in \Omega_{1}$. Since $\mathbf{b}$ is independent of $\left(Z^{5}\right)^{l}$, $\mathbf{b}_{1}$ is independent of $\left(X^{1}\right)^{l}$. For each $\omega_{1} \in \pi\left(\Omega^{\prime}\right)$, since $\left(\omega_{1}, e\left(\omega_{1}\right)\right) \in \Omega^{\prime}, r\left(\mathbf{b}_{1}\left(\omega_{1}\right)\right)=$ $r\left(\pi\left[\mathbf{b}\left(\left(\omega_{1}, e\left(\omega_{1}\right)\right)\right)\right]\right)=\pi\left(r\left[\mathbf{b}\left(\left(\omega_{1}, e\left(\omega_{1}\right)\right)\right)\right]\right)=\pi\left(r\left(\left(\omega_{1}, e\left(\omega_{1}\right)\right)\right)\right)=r\left(\omega_{1}\right)$. This shows that $X^{1}$ has $\mathbf{b}$, which is $\mathbf{b}_{1}$. Similarly, one can show that $X^{2}$ has $\mathbf{b}$ as well.

An rcll field $X: \mathbb{R}^{N} \rightarrow \mathbb{R}^{d}$ is called b-related for a fixed Borel set $G \subset \mathbb{R}^{N}$, or $G \subset \mathbb{R}_{+}^{N}$, if there exist an rcll field $X^{b}: \mathbb{R}^{N} \rightarrow \mathbb{R}^{d}$ having $\mathbf{b}$ and a constant $C \in(0, \infty)$ such that

$$
E\left\{\lambda_{d}(X(G)-F)\right\}>0 \Longrightarrow E\left\{\lambda_{d}\left(X^{b}(G)-F\right)\right\}>0
$$

for each compact $F \subset \mathbb{R}^{d}$ and $Q_{\eta}^{X} \leq C Q_{\eta}^{X^{b}}$ for each $\eta \in \mathcal{P}(G)$, where both $X^{b}$ and $C$ may depend on $G$ and $F$, but are independent of $\eta$. Obviously if $X$ has $\mathbf{b}, X$ is b-related.

It is pretty safe to say that almost every field of interest can be studied from the standpoint of the $\mathbf{b}$-condition. More importantly, the idea of $\mathbf{a} \mathbf{b}$-condition is very flexible for solving concrete problems. It imposes no restrictions on more complex fields generated from the original ones such as $k$-multiple point (time) fields (see Example 2 and Example 4 in Section 3), subordinate fields (see Example 5 of Section 3 and also Section 4 below), graphic fields, etc., which must be dealt with as the intermediate entities. This paper does not seek how to convince the reader of this claim. Instead we offer some new ideas concerning potential theory for random fields through the proofs of some potential theorems. However we will provide meaningful examples of fields having $\mathbf{b}$ later on in Section 4.

Proposition 2.2. Let $X^{1}: \mathbb{R}^{n_{1}} \rightarrow \mathbb{R}^{d}$ and $X^{2}: \mathbb{R}^{n_{2}} \rightarrow \mathbb{R}^{d}$ be independent rcll fields both continuous in probability and having $\mathbf{b}$. Assume that $X_{0}^{2}=0$ a.s. Let $G_{1} \subset \mathbb{R}^{n_{1}}$ and $G_{2} \subset \mathbb{R}^{n_{2}}$ be two Borel sets. Let $\Gamma=E\left\{\lambda_{d}\left[X^{1}\left(G_{1}\right)+X^{2}\left(G_{2}\right)\right]\right\}$ and $\mathcal{E}=\inf \left\{(2 \pi)^{-d} \int_{\mathbb{R}^{d}} Q_{\mu_{1}}^{X^{1}}(\xi) Q_{\mu_{2}}^{X^{2}}(\xi) d \xi: \mu_{1} \in \mathcal{P}\left(G_{1}\right), \mu_{2} \in \mathcal{P}\left(G_{2}\right)\right\}$. If $\Gamma>0$, then $\mathcal{E} \leq 2 / \Gamma$.

Proof. We only have to assume that both $G_{1}$ and $G_{2}$ are compact and

$$
\Gamma_{R}=E\left\{\lambda_{d}\left[\left(X^{1}\left(G_{1}\right)+X^{2}\left(G_{2}\right)\right) \cap B(0, R)\right]\right\}>0
$$


for all sufficiently large $R \in(0, \infty)$, where and throughout $B(x, r) \subset \mathbb{R}^{m}$ will denote the open ball of radius $r$ with center at $x$.

Define $Y_{t}=X_{t_{1}}^{1}+X_{t_{2}}^{2}, t=\left(t_{1}, t_{2}\right), t_{1} \in \mathbb{R}^{n_{1}}, t_{2} \in \mathbb{R}^{n_{2}}, n=n_{1}+n_{2}, G=G_{1} \times G_{2}$, and $G^{\delta}=G_{1}^{\delta} \times G_{2}^{\delta}$ for $\delta>0$, where $G_{i}^{\delta} \subset \mathbb{R}^{n_{i}}$ is the closed $\delta$-enlargement of $G_{i}$, that is, the smallest compact set such that for each point $s=\left(s_{1}, \cdots, s_{n_{1}}\right) \in G_{i}$, $\left[s_{1}, s_{1}+\delta\right] \times \cdots \times\left[s_{n_{1}}, s_{n_{1}}+\delta\right] \subset G_{i}^{\delta}$.

In the following we will use the space $(\Omega, \mathcal{F})$ of rcll functions from $\mathbb{R}^{n}$ into $\mathbb{R}^{d}$ endowed with the canonical Skorohod topology. Let $P$ be the complete probability measure on $\Omega$ given by $Y$, i.e., the law of $Y$, where we assume that $Y$ is in the canonical form. Let $P^{x}, x \in \mathbb{R}^{d}$, be the probability measure on $(\Omega, \mathcal{F})$ given by the path spatial shift $P^{x}\{\bullet\}=P^{0}\{x+\bullet\}$, where $x+A=\{x+\omega: \omega \in A\}, A \in \mathcal{F}$, $P^{0}=P$, and $x+\omega \in \Omega$ is defined by $(x+\omega)(t)=x+\omega(t), t \in \mathbb{R}^{N}$. This gives rise to a $\sigma$-finite measure $P_{\lambda}$ on $(\Omega, \mathcal{F})$ given by $P_{\lambda}(\bullet)=\int_{\mathbb{R}^{d}} P^{x}(\bullet) d x$. To see $\sigma$ finiteness of $P_{\lambda}$, let $\mathbb{R}^{d}=\bigcup_{i} E_{i}$ where $\lambda_{d}\left(E_{i}\right)<\infty$. Define $A_{i}=\left\{\omega: \omega(0) \in E_{i}\right\}$. Then $\Omega=\bigcup_{i} A_{i}$ and $P_{\lambda}\left(A_{i}\right)=\lambda_{d}\left(E_{i}\right)$. (Recall that if $P_{t}(x, A)$ is the transition function of a Markov process in $\mathbb{R}^{d}$, then each $\sigma$-finite measure $\mu$ in $\mathbb{R}^{d}$ gives rise to a measure $\mu P_{t}$ on $\mathbb{R}^{d}$ by $\mu P_{t}(A)=\int_{\mathbb{R}^{d}} P_{t}(x, A) \mu(d x)$. In potential theory for Markov processes, the supersript notation $P^{\mu}$ is often used. So the construction is similar, but on two totally different spaces: $\mathbb{R}^{d}$ and $\Omega$.)

By Lemma 2.1 (cf. the field $Z^{4}$ ), $Y$ has b. The domain $J\left(l_{1}\right)$ for $Y^{l_{1}}$ can be $\left[-l_{1}, l_{1}\right)^{n},\left[0, l_{1}\right)^{n},\left[0, l_{1}\right)^{n_{1}} \times\left[-l_{1}, l_{1}\right)^{n_{2}},\left(\left[-l_{1}, l_{1}\right) \times \mathbb{R}^{n_{1}-1}\right) \times\left(\left[-l_{1}, l_{1}\right) \times \mathbb{R}^{n_{2}-1}\right)$, $\left(\left[-l_{1}, l_{1}\right) \times \mathbb{R}^{n_{1}-1}\right) \times\left[0, l_{1}\right)^{n_{2}},\left(\left[-l_{1}, l_{1}\right) \times \mathbb{R}^{n_{1}-1}\right) \times\left[-l_{1}, l_{1}\right)^{n_{2}}$, etc. In any case, we fix a large $l_{1}$ satisfying $G^{\delta} \subset J\left(l_{1}\right)$. Let $\mathbf{b}: \Omega \rightarrow \Omega$, or $\mathbf{b}: \Omega \rightarrow \Omega^{*}$, where $\Omega^{*}$ is the space of rcll functions from $\mathbb{R}_{+}^{n_{1}} \times \mathbb{R}^{n_{2}}$ into $\mathbb{R}^{d}$, or $\mathbf{b}: \Omega \rightarrow \Omega_{+}$be the measurable mapping such that (1) $\mathbf{b}$ is independent of $Y^{l_{1}}$ and (2) for some event $\Omega^{\prime} \subset \Omega$ with $r\left(\Omega^{\prime}\right)=\Omega_{l_{1}}, r\left[\mathbf{b}\left(\omega^{\prime}\right)\right]=r\left(\omega^{\prime}\right)$ for each $\omega^{\prime} \in \Omega^{\prime}$, where $\Omega_{l_{1}}$ is the space of rcll functions from $J\left(l_{1}\right)$ into $\mathbb{R}^{d}$, the sample space of $Y^{l_{1}}$, and $r: \Omega \rightarrow \Omega_{l_{1}}$ is the restriction mapping. Let the probability measure $P_{l_{1}}$ be the natural restriction of $P$ to $\Omega_{l_{1}}$, i.e., the law of $Y^{l_{1}}$. We then define the $\sigma$-finite measure $\left[P_{l_{1}}\right]_{\lambda}$ on $\Omega_{l_{1}}$ in an analogous way. Consider the event

$$
A^{\delta}=\left\{\omega \in \Omega: \omega(t) \in B(0, \delta) \text { for some } t \in G^{\delta}, \omega(0) \in B(0, R)\right\} .
$$

The fact that $\omega$ is right continuous and $G_{i}^{\delta}$ is the closed $\delta$-enlargement of the compact set $G_{i}$ implies that

$$
A^{\delta}=\left\{\omega \in \Omega: \omega(t) \in B(0, \delta) \text { for some } t \in G^{\delta} \cap \mathbb{Q}^{n}, \omega(0) \in B(0, R)\right\},
$$

where as always $\mathbb{Q}$ stands for the rational field. (This also shows that $A^{\delta} \in \mathcal{F}$.) Note that $Y$ and $Y^{l_{1}}$ are both in the canonical form. By Fubini's theorem,

$$
P_{\lambda}\left\{A^{\delta}\right\}=E\left\{\lambda_{d}\left(\left[Y\left(G^{\delta}\right)-B(0, \delta)\right] \cap B(0, R)\right)\right\},
$$

which implies that

$$
\infty>\lambda_{d}[B(0, R)] \geq P_{\lambda}\left\{A^{\delta}\right\} \geq E\left\{\lambda_{d}(Y(G) \cap B(0, R))\right\}>0 .
$$

Clearly,

$$
r\left(A^{\delta}\right)=\left\{\omega \in \Omega_{l_{1}}: \omega(t) \in B(0, \delta) \text { for some } t \in G^{\delta}, \omega(0) \in B(0, R)\right\} .
$$


Since $r\left(\Omega^{\prime}\right)=\Omega_{l_{1}}, r\left(\Omega^{\prime} \cap A^{\delta}\right)=r\left(A^{\delta}\right)$. Let $S \subset A^{\delta}$ be any event. Recall that $G^{\delta} \subset J\left(l_{1}\right)$. Let $E_{l_{1}}$ be the expectation for $P_{l_{1}}$. We have

$$
\begin{gathered}
P_{\lambda}(S)=E\left\{\lambda_{d}\left(\left[Y\left(G^{\delta}\right)-B(0, \delta)\right] \cap B(0, R)\right) 1_{S}\right\} \\
=E_{l_{1}}\left\{\lambda_{d}\left(\left[Y^{l_{1}}\left(G^{\delta}\right)-B(0, \delta)\right] \cap B(0, R)\right) 1_{r(S)}\right\}=\left[P_{l_{1}}\right]_{\lambda}(r(S)) .
\end{gathered}
$$

In particular, $P_{\lambda}\left\{A^{\delta}\right\}=\left[P_{l_{1}}\right]_{\lambda}\left\{r\left(A^{\delta}\right)\right\}$. Set $\widetilde{A}^{\delta}=\Omega^{\prime} \cap A^{\delta}$. Since $r\left(\widetilde{A}^{\delta}\right)=r\left(A^{\delta}\right)$,

$$
P_{\lambda}\left\{\widetilde{A}^{\delta}\right\}=\left[P_{l_{1}}\right]_{\lambda}\left\{r\left(\widetilde{A}^{\delta}\right)\right\}=\left[P_{l_{1}}\right]_{\lambda}\left\{r\left(A^{\delta}\right)\right\}=P_{\lambda}\left\{A^{\delta}\right\} .
$$

For each $\omega \in A^{\delta}$, denote by $\zeta_{\omega}^{\delta}$ the set of the points $u \in G^{\delta} \cap \mathbb{Q}^{n}$ such that $|\omega(u)|<$ $\delta$. Clearly, $\zeta_{\omega}^{\delta}$ is a countably infinite set and the set $\zeta_{\omega}^{\delta}$ remains the same for both $\omega \in A^{\delta}$ and $\omega \in r\left(A^{\delta}\right)$. Note that the set $r\left(A^{\delta}\right) \subset \Omega_{l_{1}}$ is measuable. Thus, using the Skorohod topology on $\Omega_{l_{1}}$, we are able to define a Borel mapping $\theta: \Omega_{l_{1}} \rightarrow G^{\delta} \cap \mathbb{Q}^{n}$ such that for each $\omega \in r\left(A^{\delta}\right), \theta(\omega) \in \zeta_{\omega}^{\delta}$, where the countable set $G^{\delta} \cap \mathbb{Q}^{n}$ has its discrete topology. We can construct such a $\theta$ with more regularity properties (for proving our proposition only). Recall that $X_{0}^{2}=0$ a.s. Let $\Omega_{l_{1}}^{1}+A_{l_{1}}^{2}=\left\{\omega_{1}+\omega_{2}\right.$ : $\left.\omega_{1} \in \Omega_{l_{1}}^{1}, \omega_{2} \in A_{l_{1}}^{2}\right\}$, where $A_{l_{1}}^{2}=\left\{\omega_{2} \in \Omega_{l_{1}}^{2}: \omega_{2}(0)=0\right\}$. If $\omega_{1}+\omega_{2}=\omega_{1}^{\prime}+\omega_{2}^{\prime}$, where $\omega_{1}, \omega_{1}^{\prime} \in \Omega_{l_{1}}^{1}, \omega_{2}, \omega_{2}^{\prime} \in A_{l_{1}}^{2}$, then $\omega_{1}\left(t_{1}\right)+\omega_{2}(0)=\omega_{1}^{\prime}\left(t_{1}\right)+\omega_{2}^{\prime}(0)$. Since $\omega_{2}(0)=\omega_{2}^{\prime}(0)=0, \omega_{1}\left(t_{1}\right)=\omega_{1}^{\prime}\left(t_{1}\right)$, i.e., $\omega_{1}=\omega_{1}^{\prime}$. Consequently, $\omega_{2}=\omega_{2}^{\prime}$. Thus it is possible to construct $\theta$ and measurable mappings $\theta_{1}: \Omega_{l_{1}}^{1} \rightarrow G_{1}^{\delta} \cap \mathbb{Q}^{n_{1}}, \theta_{2}: A_{l_{1}}^{2} \rightarrow$ $G_{2}^{\delta} \cap \mathbb{Q}^{n_{2}}$ such that for each $\omega \in r\left(A^{\delta}\right), \theta(\omega) \in \zeta_{\omega}^{\delta}$, and for each $\omega=\omega_{1}+\omega_{2} \in$ $\Omega_{l_{1}}^{1}+A_{l_{1}}^{2}, \theta(\omega)=\left(\theta_{1}\left(\omega_{1}\right), \theta_{2}\left(\omega_{2}\right)\right)$. The restriction mapping $r$ from $\Omega$ to $\Omega_{l_{1}}$ is clearly measurable. Thus, $\phi=\theta \circ r: \Omega \rightarrow G^{\delta} \cap \mathbb{Q}^{n}$ is a measurable mapping. Finally, for each $\omega^{\prime} \in \widetilde{A}^{\delta}$,

$$
\phi\left(\mathbf{b}\left(\omega^{\prime}\right)\right)=\theta\left(r\left(\mathbf{b}\left(\omega^{\prime}\right)\right)\right)=\theta\left(r\left(\omega^{\prime}\right)\right) \in \zeta_{r\left(\omega^{\prime}\right)}^{\delta}=\zeta_{\omega^{\prime}}^{\delta}
$$

since $r\left(\omega^{\prime}\right) \in r\left(A^{\delta}\right)$. Define

$$
T^{\delta}=\phi \circ \mathbf{b} .
$$

It is immediate that $T^{\delta}$ is a random variable defined on $\Omega$ taking values in the countable set $G^{\delta} \cap \mathbb{Q}^{n}$ satisfying (i) $T^{\delta}$ is independent of $Y^{l_{1}}$ and (ii)

$$
T^{\delta}(\omega) \in \zeta_{\omega}^{\delta} \text {, for each } \omega \in \widetilde{A}^{\delta} .
$$

Define

$$
\mu^{\delta}(\bullet)=\frac{P_{\lambda}\left\{T^{\delta} \in \bullet, \widetilde{A}^{\delta}\right\}}{P_{\lambda}\left\{\widetilde{A}^{\delta}\right\}}
$$

which is a probability measure on $G^{\delta}$ since $P_{\lambda}\left\{\widetilde{A}^{\delta}\right\}=P_{\lambda}\left\{A^{\delta}\right\} \in(0, \infty)$ by $(2.5)$ and (2.6). Indeed we know more about $\mu^{\delta}$. Set $T^{\delta}(\omega)=\left(T_{1}^{\delta}(\omega), T_{2}^{\delta}(\omega)\right)$. Let $A^{2}=$ $\left\{\omega_{2} \in \Omega^{2}: \omega_{2}(0)=0\right\}$. Without loss of generality we can assume that $\mathbf{b}_{2}$ satisfies $\mathbf{b}_{2}\left(\omega_{2}\right) \in A^{2}$ for $\omega_{2} \in A^{2}$. Similarly, if $\omega_{1}+\omega_{2}=\omega_{1}^{\prime}+\omega_{2}^{\prime}$, where $\omega_{1}, \omega_{1}^{\prime} \in \Omega^{1}, \omega_{2}, \omega_{2}^{\prime} \in$ $A^{2}$, then $\omega_{1}=\omega_{1}^{\prime}$ and $\omega_{2}=\omega_{2}^{\prime}$. The properties of $\theta$ and of $\mathbf{b}$ imply that

$$
\begin{array}{r}
T_{1}^{\delta}(\omega)=\theta_{1} \circ r \circ \mathbf{b}_{1}\left(\omega_{1}\right) \in G_{1}^{\delta} \cap \mathbb{Q}^{n_{1}}, T_{2}^{\delta}(\omega)=\theta_{2} \circ r \circ \mathbf{b}_{2}\left(\omega_{2}\right) \\
\forall G_{2}^{\delta} \cap \mathbb{Q}^{n_{2}}, \\
\forall \omega=\omega_{1}+\omega_{2} \in \Omega^{2}+A^{2} .
\end{array}
$$

Since $\mathbf{b}_{1}$ and $\mathbf{b}_{2}$ are independent, i.e., $X^{1}$ and $X^{2}$ are independent, (2.10) implies that $T_{1}^{\delta}$ and $T_{2}^{\delta}$ are independent. Since $P_{\lambda}\left(\widetilde{A}^{\delta}\right)=\left[P_{l_{1}}\right]_{\lambda}\left(r\left(A^{\delta}\right)\right)$ and since $T_{1}^{\delta}$ and 
$T_{2}^{\delta}$ are both independent of $Y_{t}, t \in G^{\delta} \subset J\left(l_{1}\right)$, we obtain

$$
\mu^{\delta}=\mu_{1}^{\delta} \times \mu_{2}^{\delta}
$$

for some $\mu_{1}^{\delta} \in \mathcal{P}\left(G_{1}^{\delta}\right)$ and $\mu_{2}^{\delta} \in \mathcal{P}\left(G_{2}^{\delta}\right)$.

Let $\mathbf{x}: \mathbb{R}^{N} \rightarrow \mathbb{R}^{d}$ be an rcll field. For $s, t \in \mathbb{R}^{N}$ and $f$ with $f, \hat{f} \in L^{1}$, we define

$$
P_{s, t} f(x)=E\left[f\left(\mathbf{x}_{t}-\mathbf{x}_{s}+x\right)\right], x \in \mathbb{R}^{d} .
$$

Using the inversion formula for convolution yields

$$
P_{s, t} f(x)=(2 \pi)^{-d} \int_{\mathbb{R}^{d}} e^{i x \cdot \xi} \hat{f}(-\xi) E e^{i \xi \cdot\left(\mathbf{x}_{t}-\mathbf{x}_{s}\right)} d \xi, x \in \mathbb{R}^{d} .
$$

Define for each $\varepsilon>0, f^{\varepsilon}(x)=\left(2 \pi \varepsilon^{2}\right)^{-d / 2} e^{-\frac{|x|^{2}}{2 \varepsilon^{2}}}, x \in \mathbb{R}^{d}, P_{s, t} f^{\varepsilon}(x)=E\left[f^{\varepsilon}\left(Y_{t}-\right.\right.$ $\left.\left.Y_{s}+x\right)\right], s, t \in \mathbb{R}^{n}, x \in \mathbb{R}^{d}, N(v, x)=\int_{\mathbb{R}^{n}} P_{v, t} f^{\varepsilon}(x) \mu^{\delta}(d t), v \in \mathbb{R}^{n}, x \in \mathbb{R}^{d}$, and $H(s, t, \xi)=E e^{i \xi \cdot\left(Y_{s}-Y_{t}\right)}: \mathbb{R}^{n} \times \mathbb{R}^{n} \times \mathbb{R}^{d} \rightarrow \mathbb{C}$. Since $Y$ is continuous in probability, $H$ is a bounded continuous function. Since $\widehat{f^{\varepsilon}}(-\xi)=e^{-\frac{\varepsilon^{2}}{2}|\xi|^{2}}$ and since we are allowed to interchange the order of integration owing to the integration factor $e^{-\frac{\varepsilon^{2}}{2}|\xi|^{2}}$, by (2.12) we have, for any $v \in \mathbb{R}^{n}$,

$$
\int_{\mathbb{R}^{n}} P_{v, t} f^{\varepsilon}(0) \mu^{\delta}(d t)=(2 \pi)^{-d} \int_{\mathbb{R}^{n}} \int_{\mathbb{R}^{d}} e^{-\frac{\varepsilon^{2}}{2}|\xi|^{2}} H(u, v, \xi) d \xi \mu^{\delta}(d u) .
$$

Note that $f^{\varepsilon}$ is Lipschitz continuous. Let $D(\varepsilon)$ be the Lipschitz constant of $f^{\varepsilon}$. By the definition of $P_{s, t} f^{\varepsilon}$, we have

$$
D(\varepsilon) \delta+\inf _{|z| \leq \delta} P_{v, t} f^{\varepsilon}(z) \geq P_{v, t} f^{\varepsilon}(0) .
$$

Since $\mu^{\delta}$ is a probability measure, we have

$$
\int_{\mathbb{R}^{n}} \inf _{|z| \leq \delta} P_{v, t} f^{\varepsilon}(z) \mu^{\delta}(d t) \geq \int_{\mathbb{R}^{n}} P_{v, t} f^{\varepsilon}(0) \mu^{\delta}(d t)-D(\varepsilon) \delta .
$$

Let $x$ be any vector in $\mathbb{R}^{d}$ such that $|x| \leq \delta$. Then $P_{v, t} f^{\varepsilon}(x) \geq \inf _{|z| \leq \delta} P_{v, t} f^{\varepsilon}(z)$. It follows that for any $|x|<\delta$ and $v \in \mathbb{R}^{n}$,

$N(v, x)=\int_{\mathbb{R}^{n}} P_{v, t} f^{\varepsilon}(x) \mu^{\delta}(d t) \geq(2 \pi)^{-d} \int_{\mathbb{R}^{n}} \int_{\mathbb{R}^{d}} e^{-\frac{\varepsilon^{2}}{2}|\xi|^{2}} H(u, v, \xi) d \xi \mu^{\delta}(d u)-D(\varepsilon) \delta$.

Recall that $Y$ is in the canonical form, i.e., $Y_{u}(\omega)=\omega(u)$ for each $\omega \in \Omega$ and each $u \in \mathbb{R}^{n}$. By (2.8),

$$
\left|Y_{T^{\delta}(\omega)}(\omega)\right|<\delta \text { for each } \omega \in \widetilde{A}^{\delta} .
$$

Thus, taking $v=T^{\delta}$ and $x=Y_{T^{\delta}}$ in (2.13) yields

$$
1_{\widetilde{A}^{\delta}} N\left(T^{\delta}, Y_{T^{\delta}}\right) \geq 1_{\widetilde{A}^{\delta}}\left\{(2 \pi)^{-d} \int_{\mathbb{R}^{n}} \int_{\mathbb{R}^{d}} e^{-\frac{\varepsilon^{2}}{2}|\xi|^{2}} H\left(u, T^{\delta}, \xi\right) d \xi \mu^{\delta}(d u)-D(\varepsilon) \delta\right\},
$$

which implies that

$$
1_{\widetilde{A}^{\delta}} D(\varepsilon) \delta+N\left(T^{\delta}, Y_{T^{\delta}}\right) \geq 1_{\widetilde{A}^{\delta}}(2 \pi)^{-d} \int_{\mathbb{R}^{n}} \int_{\mathbb{R}^{d}} e^{-\frac{\varepsilon^{2}}{2}|\xi|^{2}} H\left(u, T^{\delta}, \xi\right) d \xi \mu^{\delta}(d u) .
$$

Applying the inequality $(a+b)^{2} \leq 2 a^{2}+2 b^{2}$ then gives us

$$
1_{\widetilde{A}^{\delta}} 2[D(\varepsilon) \delta]^{2}+2 N^{2}\left(T^{\delta}, Y_{T^{\delta}}\right) \geq 1_{\widetilde{A}^{\delta}}(2 \pi)^{-2 d}\left(\int_{\mathbb{R}^{n}} \int_{\mathbb{R}^{d}} e^{-\frac{\varepsilon^{2}}{2}|\xi|^{2}} H\left(u, T^{\delta}, \xi\right) d \xi \mu^{\delta}(d u)\right)^{2} .
$$


Let $E_{\lambda}$ denote the expectation operator with respect to $P_{\lambda}$ for real-valued random variables $\mathbf{w}$ defined on $\Omega$. One can check that $E_{\lambda}(\mathbf{w})=\int_{\mathbb{R}^{d}} E^{x}(\mathbf{w}) d x$ for nonnegative w. Taking $E_{\lambda}$ on both sides of (2.15) followed by Jensen's inequality, since $\mu^{\delta}$ is a probability measure, and interchanging order of integration thanks to the integrable factor $e^{-\frac{\varepsilon^{2}}{2}|\xi|^{2}}$ turn (2.15) into

$2[D(\varepsilon) \delta]^{2} P_{\lambda}\left(A^{\delta}\right)+2 E_{\lambda}\left\{N^{2}\left(T^{\delta}, Y_{T^{\delta}}\right)\right\} \geq(2 \pi)^{-2 d}\left\{\int_{\mathbb{R}^{d}} e^{-\frac{\varepsilon^{2}}{2}|\xi|^{2}} Q_{\mu^{\delta}}^{Y}(\xi) d \xi\right\}^{2} P_{\lambda}\left(A^{\delta}\right)$.

(Recall that $P_{\lambda}\left(\widetilde{A}^{\delta}\right)=P_{\lambda}\left(A^{\delta}\right)$ by (2.6).) Using (2.5) we have

$2[D(\varepsilon) \delta]^{2} \lambda_{d}[B(0, R)]+2 E_{\lambda}\left\{N^{2}\left(T^{\delta}, Y_{T^{\delta}}\right)\right\} \geq(2 \pi)^{-2 d}\left\{\int_{\mathbb{R}^{d}} e^{-\frac{\varepsilon^{2}}{2}|\xi|^{2}} Q_{\mu^{\delta}}^{Y}(\xi) d \xi\right\}^{2} \Gamma_{R}$.

Right now the issue that exists is how to change the term $E_{\lambda}\left[N^{2}\left(T^{\delta}, Y_{T^{\delta}}\right)\right]$. Let $\left\{\theta_{1}, \theta_{2}, \cdots, \theta_{k}, \cdots\right\} \subset\left[G^{\delta} \cap \mathbb{Q}^{n}\right]$ be the possible values of $T^{\delta}$ with the probability $\pi_{k}$ under $P^{0}(=P)$ that $T^{\delta}$ takes $\theta_{k}$. Since $T^{\delta}$ is independent of $Y^{l_{1}}, T^{\delta}$ is independent of $Y^{l_{1}}+x$ as well for all $x \in \mathbb{R}^{d}$ under $P^{0}(=P)$, where $Y^{l_{1}}+x$ is the field $\left\{Y_{t}+x, t \in J\left(l_{1}\right)\right\}$. Also note that $T^{\delta}$ takes values in $J\left(l_{1}\right)$. It follows that

$$
\begin{gathered}
E_{\lambda}\left\{\left[N\left(T^{\delta}, Y_{T^{\delta}}\right)\right]^{2}\right\}=\int_{\mathbb{R}^{d}} E^{x}\left\{\left[N\left(T^{\delta}, Y_{T^{\delta}}\right)\right]^{2}\right\} d x \\
=\int_{\mathbb{R}^{d}} E\left\{\left[N\left(T^{\delta}, Y_{T^{\delta}}+x\right)\right]^{2}\right\} d x=\int_{\mathbb{R}^{d}} \sum_{k=1}^{\infty} \pi_{k} E\left\{\left[N\left(\theta_{k}, Y_{\theta_{k}}+x\right)\right]^{2}\right\} d x \\
=\sum_{k=1}^{\infty} \pi_{k} \int_{\mathbb{R}^{d}} E\left\{\left[N\left(\theta_{k}, Y_{\theta_{k}}+x\right)\right]^{2}\right\} d x=\sum_{k=1}^{\infty} \pi_{k} E_{\lambda}\left\{\left[N\left(\theta_{k}, Y_{\theta_{k}}\right)\right]^{2}\right\} .
\end{gathered}
$$

The following conditional expectation representation of $P_{s, t} f\left(Y_{s}\right)$ is of some interest.

For all $s, t \in \mathbb{R}^{n}$ and all continuous functions $f: \mathbb{R}^{d} \rightarrow \mathbb{R}_{+}$in $L^{1}$,

$$
P_{s, t} f\left(Y_{s}\right)=E_{\lambda}\left[f\left(Y_{t}\right) \mid Y_{s}\right] \quad P_{\lambda}-\text { a.s. }
$$

where the $P_{\lambda}$-null set in (2.19) can be made independent of $t$.

Verification of (2.19). For all nonnegative functions $h$, by Fubini's theorem, change of variable and the fact that the Lebesgue measure is translation-invariant,

$$
E_{\lambda}\left[h\left(Y_{t}\right)\right]=\int_{\mathbb{R}^{d}} E\left[h\left(x+Y_{t}\right)\right] d x=\int_{\mathbb{R}^{d}} h(x) d x .
$$

In particular, for all $t \in \mathbb{R}^{n}$ and Borel sets $B$ in $\mathbb{R}^{d}, P_{\lambda}\left(Y_{t} \in B\right)=\lambda_{d}(B)$. That is, under $P_{\lambda}$, the distribution of $Y_{t}$ is $\lambda_{d}$ for all $t \in \mathbb{R}^{n}$. Let $g$ be a bounded measurable function. Then

$$
E_{\lambda}\left[f\left(Y_{t}\right) g\left(Y_{s}\right)\right]=\int_{\mathbb{R}^{d}} E\left[f\left(Y_{t}+x\right) g\left(Y_{s}+x\right)\right] d x=\int_{\mathbb{R}^{d}} g(y) E\left[f\left(Y_{t}-Y_{s}+y\right)\right] d y .
$$

Since the distribution of $Y_{s}$ is $\lambda_{d}$ under $P_{\lambda},(2.19)$ follows. The assertion that there is a null set independent of $t$ is quite clear beacuse $Y$ is $\operatorname{rcll}$ and $\mathbb{Q}^{n}$ is dense in $\mathbb{R}^{n}$. 
(2.19) implies that

$$
E_{\lambda}\left\{\left[N\left(\theta_{k}, Y_{\theta_{k}}\right)\right]^{2}\right\}=E_{\lambda}\left\{\left(E_{\lambda}\left[\int_{\mathbb{R}^{n}} f^{\varepsilon}\left(Y_{t}\right) \mu^{\delta}(d t) \mid Y_{\theta_{k}}\right]\right)^{2}\right\} .
$$

It follows from the conditional Jensen's inequality under $P_{\lambda}$ that

$$
E_{\lambda}\left\{\left[N\left(\theta_{k}, Y_{\theta_{k}}\right)\right]^{2}\right\} \leq E_{\lambda}\left\{\left[\int_{\mathbb{R}^{n}} f^{\varepsilon}\left(Y_{t}\right) \mu^{\delta}(d t)\right]^{2}\right\} .
$$

(2.18) and (2.20) imply that

$$
E_{\lambda}\left\{\left[N\left(T^{\delta}, Y_{T^{\delta}}\right)\right]^{2}\right\} \leq E_{\lambda}\left\{\left[\int_{\mathbb{R}^{n}} f^{\varepsilon}\left(Y_{t}\right) \mu^{\delta}(d t)\right]^{2}\right\} .
$$

Next, we show that

$$
E_{\lambda}\left\{\left[\int_{\mathbb{R}^{n}} f^{\varepsilon}\left(Y_{t}\right) \mu^{\delta}(d t)\right]^{2}\right\}=(2 \pi)^{-d} \int_{\mathbb{R}^{d}} e^{-\varepsilon^{2}|\xi|^{2}} Q_{\mu^{\delta}}^{Y}(\xi) d \xi .
$$

Proof of (2.22). As in the proof of (2.19), we find that for all $s, t \in \mathbb{R}^{n}$,

$$
E_{\lambda}\left\{f^{\varepsilon}\left(Y_{s}\right) f^{\varepsilon}\left(Y_{t}\right)\right\}=\int_{\mathbb{R}^{d}}\left(P_{s, t} f^{\varepsilon}(y)\right) f^{\varepsilon}(y) d y .
$$

By (2.12) and Fubini's theorem, thanks to $f^{\varepsilon}, \widehat{f^{\varepsilon}} \in L^{2}$,

$$
\begin{aligned}
& E_{\lambda}\left\{f^{\varepsilon}\left(Y_{s}\right) f^{\varepsilon}\left(Y_{t}\right)\right\}=\int_{\mathbb{R}^{d}}\left(P_{s, t} f^{\varepsilon}(y)\right) f^{\varepsilon}(y) d y \\
& =\int_{\mathbb{R}^{d}}\left((2 \pi)^{-d} \int_{\mathbb{R}^{d}} e^{i y \cdot \xi} \widehat{f^{\varepsilon}}(-\xi) E e^{i \xi \cdot\left(Y_{t}-Y_{s}\right)} d \xi\right) f^{\varepsilon}(y) d y \\
& =(2 \pi)^{-d} \int_{\mathbb{R}^{d}}\left|\widehat{f^{\varepsilon}}(\xi)\right|^{2} E e^{i \xi \cdot\left(Y_{t}-Y_{s}\right)} d \xi .
\end{aligned}
$$

Thus, by Fubini's theorem,

$$
\begin{aligned}
& E_{\lambda}\left\{\left[\int_{\mathbb{R}^{n}} f^{\varepsilon}\left(Y_{t}\right) \mu^{\delta}(d t)\right]^{2}\right\} \\
& =E_{\lambda}\left\{\int_{\mathbb{R}^{n}} \int_{\mathbb{R}^{n}} f^{\varepsilon}\left(Y_{s}\right) f^{\varepsilon}\left(Y_{t}\right) \mu^{\delta}(d s) \mu^{\delta}(d t)\right\} \\
& =\int_{\mathbb{R}^{n}} \int_{\mathbb{R}^{n}} E_{\lambda}\left\{f^{\varepsilon}\left(Y_{s}\right) f^{\varepsilon}\left(Y_{t}\right)\right\} \mu^{\delta}(d s) \mu^{\delta}(d t) \\
& =\int_{\mathbb{R}^{n}} \int_{\mathbb{R}^{n}}\left((2 \pi)^{-d} \int_{\mathbb{R}^{d}}\left|\widehat{f^{\varepsilon}}(\xi)\right|^{2} E e^{i \xi \cdot\left(Y_{t}-Y_{s}\right)} d \xi\right) \mu^{\delta}(d s) \mu^{\delta}(d t) \\
& =(2 \pi)^{-d} \int_{\mathbb{R}^{d}}\left|\widehat{f^{\varepsilon}}(\xi)\right|^{2} Q_{\mu^{\delta}}^{Y}(\xi) d \xi=(2 \pi)^{-d} \int_{\mathbb{R}^{d}} e^{-\varepsilon^{2}|\xi|^{2}} Q_{\mu^{\delta}}^{Y}(\xi) d \xi .
\end{aligned}
$$

$(2.22)$ is proved. 
Note that $e^{-\varepsilon^{2}|\xi|^{2}} \leq e^{-\frac{\varepsilon^{2}}{2}|\xi|^{2}}$. It follows from (2.21), (2.22) and (2.17) that

$$
\begin{gathered}
2[D(\varepsilon) \delta]^{2} \lambda_{d}[B(0, R)]+2(2 \pi)^{-d} \int_{\mathbb{R}^{d}} e^{-\frac{\varepsilon^{2}}{2}|\xi|^{2}} Q_{\mu^{\delta}}^{Y}(\xi) d \xi \\
\geq(2 \pi)^{-2 d}\left\{\int_{\mathbb{R}^{d}} e^{-\frac{\varepsilon^{2}}{2}|\xi|^{2}} Q_{\mu^{\delta}}^{Y}(\xi) d \xi\right\}^{2} \Gamma_{R} .
\end{gathered}
$$

Since $X^{1}$ and $X^{2}$ are independent and since $\mu^{\delta}=\mu_{1}^{\delta} \times \mu_{2}^{\delta}, Q_{\mu^{\delta}}^{Y}(\xi)=Q_{\mu_{1}^{\delta}}^{X^{1}}(\xi) Q_{\mu_{2}^{\delta}}^{X^{2}}(\xi)$. We have now arrived at

$$
\begin{gathered}
2[D(\varepsilon) \delta]^{2} c_{d, R}+2(2 \pi)^{-d} \int_{\mathbb{R}^{d}} e^{-\frac{\varepsilon^{2}}{2}|\xi|^{2}} Q_{\mu_{1}^{\delta}}^{X^{1}}(\xi) Q_{\mu_{2}^{\delta}}^{X^{2}}(\xi) d \xi \\
\geq(2 \pi)^{-2 d}\left\{\int_{\mathbb{R}^{d}} e^{-\frac{\varepsilon^{2}}{2}|\xi|^{2}} Q_{\mu_{1}^{\delta}}^{X^{1}}(\xi) Q_{\mu_{2}^{\delta}}^{X^{2}}(\xi) d \xi\right\}^{2} \Gamma_{R},
\end{gathered}
$$

where $c_{d, R}=\lambda_{d}(B(0, R))$. Since $G_{i}^{\delta}$ is compact and since $H^{i}(s, t, \xi)=E e^{i \xi \cdot\left(X_{s}^{i}-X_{t}^{i}\right)}$ is continuous due to the fact that $X^{i}$ is continuous in probability, the standard weak convergence argument of letting $\delta \downarrow 0$ in (2.24) shows that there exist $\mu_{1} \in \mathcal{P}\left(G_{1}\right)$ and $\mu_{2} \in \mathcal{P}\left(G_{2}\right)$ such that

$$
(2 \pi)^{-d} \int_{\mathbb{R}^{d}} e^{-\frac{\varepsilon^{2}}{2}|\xi|^{2}} Q_{\mu_{1}}^{X^{1}}(\xi) Q_{\mu_{2}}^{X^{2}}(\xi) d \xi \leq \frac{2}{\Gamma_{R}} .
$$

(Get more information about the weak convergence argument in the proof of Proposition 4.1 of Section 4 below.) Letting $\varepsilon \rightarrow 0$ thanks to Fatou's lemma we obtain

$$
(2 \pi)^{-d} \int_{\mathbb{R}^{d}} Q_{\mu_{1}}^{X^{1}}(\xi) Q_{\mu_{2}}^{X^{2}}(\xi) d \xi \leq \frac{2}{\Gamma_{R}} .
$$

Since $\Gamma_{R} \rightarrow \Gamma$ as $R \rightarrow \infty, \mathcal{E} \leq 2 / \Gamma$ follows from (2.26).

Theorem 2.3. Let $X: \mathbb{R}^{N} \rightarrow \mathbb{R}^{d}$ be any rcll field. Fix a Borel set $G \subset \mathbb{R}^{N}$. Let $F \subset \mathbb{R}^{d}$ be any Borel set.

(i) $[\Gamma(F)]^{-1} \leq \mathcal{E}(F)$.

(ii) If $X$ is continuous in probability and has $\mathbf{b}$, then $\mathcal{E}(F) / 2 \leq[\Gamma(F)]^{-1}$.

(iii) If $X$ is $\mathbf{b}$-related for $G$ with $X^{b}$ being continuous in probability, then $\Gamma(F)>$ $0 \Longrightarrow \mathcal{E}(F)<\infty$.

Proof. Set $Y_{(t, s)}=X_{t}+V_{s}, t \in \mathbb{R}^{N}, s \in \mathbb{R}^{d}$, where $V: \mathbb{R}^{d} \rightarrow \mathbb{R}^{d}$ is the deterministic field $V_{s}=-s$. Note that $Q_{\mu}^{V}(\xi)=|\hat{\mu}(\xi)|^{2}$ for any probability measure $\mu$ in $\mathbb{R}^{d}$.

(i) If $\mathcal{E}(F)<\infty$, then $\int_{\mathbb{R}^{d}}|\hat{\mu}(\xi)|^{2} Q_{\eta}^{X}(\xi) d \xi<\infty$ for some $\mu \in \mathcal{P}(F), \eta \in \mathcal{P}(G)$. By Proposition 4.2 of Section 4 below,

$$
\begin{gathered}
E\left\{\lambda_{d}(X(G)-F)\right\}=E\left\{\lambda_{d}(Y(G \times F))\right\} \\
\geq(2 \pi)^{d}\left(\int_{\mathbb{R}^{d}} Q_{\eta \times \mu}^{Y}(\xi) d \xi\right)^{-1}=(2 \pi)^{d}\left(\int_{\mathbb{R}^{d}}|\hat{\mu}(\xi)|^{2} Q_{\eta}^{X}(\xi) d \xi\right)^{-1},
\end{gathered}
$$

which implies that $[\Gamma(F)]^{-1} \leq \mathcal{E}(F)$.

(ii) If $\Gamma(F)=0$, then $\mathcal{E}(F)=\infty$ by (i). Plainly, $V$ is an rcll field that is continuous in probability and happens to have stationary increments. It is totally trivial to construct $\mathbf{b}$ for a deterministic rcll field. Let $\theta$ be a deterministic rcll mapping from $\mathbb{R}^{N}$ into $\mathbb{R}^{d}$, namely a deterministic field. Let $\mathbf{b}(\omega)=\omega, \omega \in \Omega$. Let $\Omega^{\prime}=\Omega$. Obviously, for any kind of domain for $\theta^{l}, \mathbf{b}$ is independent of $\theta^{l}$ and $r\left(\Omega^{\prime}\right)=\Omega_{l}$, and $r(\mathbf{b}(\omega))=r(\omega)$ for each $\omega \in \Omega^{\prime}$. [Remark: Proposition 2.2 implies 
that if $\theta^{i}$ are two $\mathbb{R}^{d}$-valued continuous functions with $\theta^{2}(0)=0$, then given any Borel sets $G_{i} \subset \mathbb{R}^{n_{i}}$,

$$
\begin{aligned}
\lambda_{d}\left(\theta^{1}\left(G_{1}\right)+\theta^{2}\left(G_{2}\right)\right)>0 \Longrightarrow & \int_{\mathbb{R}^{d}} Q_{\eta_{1}}^{\theta^{1}}(\xi) Q_{\eta_{2}}^{\theta^{2}}(\xi) d \xi<\infty \\
& \text { for some } \eta_{1} \in \mathcal{P}\left(G_{1}\right), \eta_{2} \in \mathcal{P}\left(G_{2}\right),
\end{aligned}
$$

where $Q_{\eta_{i}}^{\theta^{i}}(\xi)=\int_{\mathbb{R}^{N}} \int_{\mathbb{R}^{N}} e^{i \xi \cdot\left(\theta_{s}^{i}-\theta_{t}^{i}\right)} \eta_{i}(d s) \eta_{i}(d t)$. In fact, conditions on $\theta^{i}$ can be weakened. The above result is still valid if $e^{i \xi \cdot\left(\theta_{s}^{i}-\theta_{t}^{i}\right)}$ can be approximated, for example, by simple functions for rcll $\theta^{i}$.]

Assume that $\Gamma(F)>0$. In Proposition 2.2, if we choose $X^{1}=X, X^{2}=V$ $\left(V_{0}=0\right), n_{1}=N, n_{2}=d, G_{1}=G$, and $G_{2}=F$, then $\mathcal{E}(F) / 2 \leq[\Gamma(F)]^{-1}$.

(iii) Let $\Gamma^{b}$ and $\mathcal{E}^{b}$ denote capacity and energy respectively for the field $X^{b}$. We can assume that $F$ is compact. We have by (ii)

$$
\Gamma(F)>0 \Longrightarrow \Gamma^{b}(F)>0 \Longrightarrow\left[\Gamma^{b}(F)\right]^{-1} \geq 2^{-1} \mathcal{E}^{b}(F) .
$$

Since $Q_{\eta}^{X} \leq C Q_{\eta}^{X^{b}}$ for each $\eta \in \mathcal{P}(G), \mathcal{E}^{b}(F) \geq C^{-1} \mathcal{E}(F)$. Thus, $\left[\Gamma^{b}(F)\right]^{-1} \geq$ $(2 C)^{-1} \mathcal{E}(F)$. If $\mathcal{E}(F)=\infty$, then $\Gamma^{b}(F)=0$, which is a contradiction.

In this paper when we say that a field $X: \mathbb{R}^{N} \rightarrow \mathbb{R}^{d}$ has stationary increments we mean that $X_{s}-X_{t}$ and $X_{s-t}$ have the same distribution for all $s, t \in \mathbb{R}^{N}$. Note that this property will force $X_{0}=0$ a.s. to hold.

Theorem 2.4. Let $X: \mathbb{R}^{N} \rightarrow \mathbb{R}^{d}$ be any rcll field. Fix a Borel set $G \subset \mathbb{R}_{+}^{N}$ satisfying $G^{o} \neq \emptyset$. Assume that $X$ is $\mathbf{b}$-related for $G$ and $X^{b}$ has stationary increments. Define

$$
\mathcal{E}_{b}^{1}(F)=\inf \left\{(2 \pi)^{-d} \int_{\mathbb{R}^{d}}|\hat{\mu}(\xi)|^{2} Q_{\kappa}^{X^{b}}(\xi) d \xi: \mu \in \mathcal{P}(F)\right\}
$$

Then for any Borel set $F \subset \mathbb{R}^{d}$,

$$
\Gamma(F)>0 \Longleftrightarrow \mathcal{E}_{b}^{1}(F)<\infty .
$$

(If $F$ is compact, $F^{o} \neq \emptyset$, and $\mathcal{E}_{b}^{1}(F)<\infty$, then $\mathcal{E}_{b}^{1}(F)=(2 \pi)^{-d} \int_{\mathbb{R}^{d}}\left|\widehat{\lambda_{F}}(\xi)\right|^{2} Q_{\kappa}^{X^{b}}(\xi) d \xi$, where $\lambda_{F}$ is the normalized Lebesgue measure on $F$.)

Proof. We have for compact $F$,

$$
\Gamma(F)>0 \Longrightarrow \Gamma^{b}(F)>0 \Longrightarrow E\left\{\lambda_{d}\left(X^{b}\left(\mathbb{R}_{+}^{N}\right)-F\right)\right\}>0 .
$$

By part (i) of Proposition 4.1 of Section 4 below, $\mathcal{E}_{b}^{1}(F)<\infty$.

To prove $\Gamma(F)>0 \Longleftarrow \mathcal{E}_{b}^{1}(F)<\infty$, we need a light technical result. Let $X: \mathbb{R}^{N} \rightarrow \mathbb{R}^{d}$ be an rcll field. Given any probability measure $\eta$ on $\mathbb{R}_{+}^{N}$, we have an occupation measure $O_{\eta}(A)=\int_{\mathbb{R}_{+}^{N}} 1\left(X_{t} \in A\right) \eta(d t)$, a random probability measure on $\mathbb{R}^{d}$. Let $I \subset G \subset \mathbb{R}_{+}^{N}$ be an $N$-dimensional compact cube. (Recall that $G^{o} \neq \emptyset$.) Let $\lambda_{I}$ be the normalized Lebesgue measure on $I$. It is easy to find that there is a constant $c \in(0, \infty)$ depending only on $I$ such that $O_{\lambda_{I}}(\bullet) \leq c O_{\kappa}(\bullet)$. Since both $O_{\lambda_{I}}$ and $O_{\kappa}$ are probability measures on $\mathbb{R}^{d}$, the standard approximation argument shows that there is some constant $c_{1} \in(0, \infty)$, depending only on $c$, such that $\left|\widehat{O}_{\lambda_{I}}(\xi)\right|^{2} \leq c_{1}\left|\widehat{O}_{\kappa}(\xi)\right|^{2}$ for all $\xi \in \mathbb{R}^{d}$, which then implies that $Q_{\lambda_{I}}^{X}(\xi) \leq$ $c_{1} Q_{\kappa}^{X}(\xi), \forall \xi \in \mathbb{R}^{d}$. Assume that for some $\mu \in \mathcal{P}(F),|\hat{\mu}(\xi)|^{2} Q_{\kappa}^{X^{b}}(\xi) \in L^{1}\left(\mathbb{R}^{d}\right)$. 
Then by the above result, $|\hat{\mu}(\xi)|^{2} Q_{\lambda_{I}}^{X^{b}}(\xi) \in L^{1}\left(\mathbb{R}^{d}\right)$. Since $Q_{\eta}^{X} \leq C Q_{\eta}^{X^{b}}$ for each $\eta \in \mathcal{P}(G),|\hat{\mu}(\xi)|^{2} Q_{\lambda_{I}}^{X}(\xi) \in L^{1}\left(\mathbb{R}^{d}\right)$. It follows from Proposition 4.2 of Section 4 below that $\Gamma(F)>0$.

\section{Markov FIELdS AND HITTING PROBABILITY}

Potential theory is the theory about hitting measure $P_{\lambda}\left\{X_{t} \in F\right.$ for some $\left.t \in G\right\}$ and NOT about hitting probability $P^{x}\left\{X_{t} \in F\right.$ for some $\left.t \in G\right\}$ for a given $x$. Only in the cases of Markov fields and Gaussian fields, if anything, may $P_{\lambda}$ be replaced by $P^{x}$. The former has the Markov property. The latter has Gaussian estimates. On the other hand, partial results on $P_{\lambda}(\bullet)=0 \Longrightarrow P^{x}(\bullet)=0$ are available for some fields such as $M$-fields. (Loosely speaking, this is the kind of field for which any joint distribution of $\left(X_{t_{1}}, \cdots, X_{t_{p}}\right)$, where $t_{1}, \cdots, t_{p} \in D$, a countable dense subset of $G$, is absolutely continuous with respect to Lebesgue measure.) In this section we will apply potential theory to obtain $P^{x}$-results for Markov fields.

Let $X: \mathbb{R}^{N} \rightarrow \mathbb{R}^{d}$ be a random field. We say that $X$ is (temporally homogeneous) Markov at $s \in \mathbb{R}_{+}^{N}$ if for any finitely many points $t_{1}, \cdots, t_{p}$ in $(0, \infty)^{N}$, the joint distribution of $\left(X_{t_{1}+s}-X_{s}, \cdots, X_{t_{p}+s}-X_{s}\right)$ conditional on $X_{s}$ and the joint distribution of $\left(X_{t_{1}}, \cdots, X_{t_{p}}\right)$ are identical. Simply put, $P\left\{X_{t_{1}+s} \in B_{1}, \cdots, X_{t_{p}+s} \in\right.$ $\left.B_{p} \mid X_{s}=x\right\}=P^{x}\left\{X_{t_{1}} \in B_{1}, \cdots, X_{t_{p}} \in B_{p}\right\} . X$ is said to be Markov if $X$ is Markov at $s$ for a.e. $s \in(0, \infty)^{N}$. Noticeably (temporally homogeneous) Markov processes are Markov fields.

We next introduce the so-called $\phi$-potential density. It turns out exactly as in the case of Markov processes that it is extremely convenient to work with Markov fields if the $\phi$-potential density assumption holds. Let $X: \mathbb{R}^{N} \rightarrow \mathbb{R}^{d}$ be a random field. Let $\phi$ be a probability density function on $\mathbb{R}_{+}^{N}$ satisfying $\phi(s)>0$ for a.e. $s \in \mathbb{R}_{+}^{N}$. Note that $\int_{\mathbb{R}_{+}^{N}} \phi(s) d s=1$. Fix $x \in \mathbb{R}^{d}$. We say that a function $y \in \mathbb{R}^{d} \mapsto$ $K^{\phi}(x, y) \in \mathbb{R}_{+}$(which only needs to be well defined a.e.) is a $\phi$-potential density of $X$ on $\mathbb{R}_{+}^{N}$ at $x$ if for all $f: \mathbb{R}^{d} \rightarrow \mathbb{R}_{+}$

$$
\int_{\mathbb{R}_{+}} \phi(s) E^{x}\left[f\left(X_{s}\right)\right] d s=\int_{\mathbb{R}^{d}} K^{\phi}(x, y) f(y) d y .
$$

Letting $f=1$ shows that $K^{\phi}(x, \cdot)$ is a probability density on $\mathbb{R}^{d}$. Note that if a function $K_{1}^{\phi}(x, \cdot)$ differs from $K^{\phi}(x, \cdot)$ only on a Lebesgue null set, then $K_{1}^{\phi}(x, \cdot)$ is also a $\phi$-potential density. Let $\mu \in \mathcal{P}\left(\mathbb{R}_{+}^{N}\right)$. Consider the random probability measure $O_{\mu}(A)=\int_{\mathbb{R}_{+}^{N}} 1\left(X_{t} \in A\right) \mu(d t), A \in \mathcal{B}\left(\mathbb{R}^{d}\right)$. We have a probability measure $E^{x} O_{\mu}(A)=\int_{\mathbb{R}_{+}^{N}} P^{x}\left(X_{t} \in A\right) \mu(d t), A \in \mathcal{B}\left(\mathbb{R}^{d}\right)$. Write $E^{x} O_{\phi}$ for $E^{x} O_{\mu}$ where $\mu(d t)=\phi(t) d t$. It is clear that if $E^{x} O_{\phi}$ is absolutely continuous with respect to Lebesgue measure, then $K^{\phi}(x, \cdot)$ is a density of $E^{x} O_{\phi}$. Let $M$ be a Markov process in $\mathbb{R}^{d}$ with the reference measure $\lambda_{d}$. Let $U^{q}, q>0$, be the $q$-resolvent kernel of $M$. Then $U^{q}(x, A)=\int_{0}^{\infty} P^{x}\left(X_{t} \in A\right) e^{-q t} d t, A \in \mathcal{B}\left(\mathbb{R}^{d}\right)$. (Note that $U^{q}\left(x, \mathbb{R}^{d}\right)=q^{-1}$. Therefore, $U^{q}(x, \cdot)$ is a finite measure. $)$ Thus, $E^{x} O_{\phi}$ corresponds to $U^{q}(x, \cdot)$ and $K^{\phi}(x, y)$ corresponds to $u^{q}(x, y)$, the density of $U^{q}(x, \cdot)$. We can call $E^{x} O_{\phi}$ the $\phi$-potential kernel of $X$ analogously. In particular, if we let $\phi(t)=\left(\prod_{j=1}^{N} q_{j}\right) e^{-\sum_{j=1}^{N} q_{j} t_{j}}, t \in \mathbb{R}_{+}^{N}, \mathbf{q}=\left(q_{1}, \cdots, q_{N}\right) \in(0, \infty)^{N}$, we can call $E^{x} O_{\phi}$ the q-potential kernel of $X$. In general, that $K^{\phi}(0, \cdot)$ exists does not imply that $K^{\phi}(x, \cdot)$ exists for a.e. $x$. In one case it does however. Let $X$ be a Markov 
field. Assume that $X_{t}$ has a density $p_{t}^{0}$ for a.e. $t \in \mathbb{R}_{+}^{N}$ under $P=P^{0}$. Since $P^{x}\left(X_{t} \in B\right)=P^{0}\left(X_{t+s} \in B \mid X_{s}=x\right)$ for a.e. $s \in(0, \infty)^{N}$ and every $x \in \mathbb{R}^{d}, X_{t}$ has a density $p_{t}^{x}$ for a.e. $t \in \mathbb{R}_{+}^{N}$ under $P^{x}$ for every $x$. (Clearly, not just for onedimensional density, if $p$-dimensional density exists under $P^{0}$, then it also exists under any $P^{x}$.) We see that $K^{\phi}(x, y)=\int_{\mathbb{R}_{+}^{N}} p_{t}^{x}(y) \phi(t) d t$ is a $\phi$-potential density at $x$ for each $x$. Let $X$ be a Markov field. Consider the operator $(E O)^{\mathbf{q}}$ defined by $(E O)^{\mathbf{q}} f(x)=\int_{\mathbb{R}_{+}^{N}} e^{-\sum_{j=1}^{N} q_{j} t_{j}} E^{x} f\left(X_{t}\right) d t, f: \mathbb{R}^{d} \rightarrow \mathbb{R}_{+}$. One can certainly use $(E O)^{\mathbf{q}}$ to define the "excessive functions" for $X$, but at present it is hard to find the "excessive functions" of any use. For example, we have not been able to use $K^{\phi}(x, y)$ to construct a closed-form energy kernel, even for a symmetric Markov field (not in the multiplicative form) or for a Markov field with stationary increments (not an additive Lévy process). In the following we will simply write $K$ for $K^{\phi}(0, \cdot)$. We will see that $K$ serves as a bridge between $P_{\lambda}$ and $P^{x}$ for Markov fields.

Lemma 3.1. Let $X: \mathbb{R}^{N} \rightarrow \mathbb{R}^{d}$ be an rcll Markov field. Let $\Gamma(F)$ be as in (1.2) with $G=(0, \infty)^{N}$. If $X$ has a $\phi$-potential density $K$, then $F$ is essentially polar $\Longrightarrow F$ is polar. Furthermore, if $K(y)>0$ for a.e. $y$, then $F$ is not essentially polar $\Longrightarrow P^{x}\left\{X_{t} \in F\right.$ for some $\left.t \in G\right\}>0$ for every $x$.

Let $X: \mathbb{R}^{N} \rightarrow \mathbb{R}^{d}$ be a field. We say that $X$ has a strong potential theory if for every Borel set $F \in \mathbb{R}^{d}$,

$$
P^{x}\left\{X_{t} \in F \text { for some } t \in G\right\}=0
$$

for some $x \Longrightarrow P^{y}\left\{X_{t} \in F\right.$ for some $\left.t \in G\right\}=0$ for every $y$,

where $G \subset \mathbb{R}_{+}^{N}$ is a fixed Borel set. For example, many Gaussian fields have strong potential theory for $G$ being an $N$-dimensional compact interval of $(0, \infty)^{N}$. Lemma 3.1 shows that any Markov field with an a.e. positive $\phi$-potential density has a strong potential theory for $G=(0, \infty)^{N}$.

Proof of Lemma 3.1. Let $B_{s}=\left(s_{1}, \infty\right) \times \cdots \times\left(s_{N}, \infty\right)$, where $s=\left(s_{1}, \cdots, s_{N}\right) \in$ $(0, \infty)^{N}$. Let $F_{x}=F-x$ and $P=P^{0}$. We have

$$
\begin{aligned}
& P^{x}\left\{X\left(B_{s}\right) \cap F \neq \emptyset\right\}=P\left\{X\left(B_{s}\right) \cap F_{x} \neq \emptyset\right\} \\
& =\int_{\mathbb{R}^{d}} P\left\{X\left(B_{s}\right) \cap F_{x} \neq \emptyset \mid X_{s}=y\right\} P\left\{X_{s} \in d y\right\} \\
& =\int_{\mathbb{R}^{d}} P\left\{\left(X\left(B_{s}\right)-X_{s}\right) \cap\left(F_{x}-y\right) \neq \emptyset \mid X_{s}=y\right\} P\left\{X_{s} \in d y\right\} \\
& =\int_{\mathbb{R}^{d}} P\left\{X(G) \cap\left(F_{x}-y\right) \neq \emptyset\right\} P\left\{X_{s} \in d y\right\}
\end{aligned}
$$

where the last equality follows from the Markov property. Assume that $X$ has a $\phi$-potential density $K$. Then

$$
\int_{\mathbb{R}_{+}^{N}} P^{x}\left\{X\left(B_{s}\right) \cap F \neq \emptyset\right\} \phi(s) d s=\int_{\mathbb{R}^{d}} P\left\{X(G) \cap\left(F_{x}-y\right) \neq \emptyset\right\} K(y) d y .
$$

If $\Gamma(F)=0$, then the RHS of (3.1) equals 0. It follows that $P^{x}\left\{X\left(B_{s}\right) \cap F \neq\right.$ $\emptyset\}=0$ for a.e. $s \in(0, \infty)^{N}$ since $\phi(s)>0$ for a.e. $s \in \mathbb{R}_{+}^{N}$, which implies that 
$P^{x}\{X(G) \cap F \neq \emptyset\}=0$. Assume that $K(y)>0$ for a.e. $y$ and $\Gamma(F)>0$. Then the RHS of (3.1) is positive, which implies that $P^{x}\left\{X\left(B_{s}\right) \cap F \neq \emptyset\right\}>0$ for some $s \in(0, \infty)^{N}$.

Lemma 3.1 and Theorems 2.3 and 2.4 imply the following theorem.

Theorem 3.2. Let $X: \mathbb{R}^{N} \rightarrow \mathbb{R}^{d}$ be any Markov field. Let $\Gamma(F)$ be as given in (1.2) with $G=(0, \infty)^{N}$ and let $\mathcal{E}(F)$ be as given in (1.4). Assume that $X$ has a $\phi$-potential density $K$. (i) If $K(y)>0$ for a.e. $y$, then $\mathcal{E}(F)<\infty \Longrightarrow P^{x}\left\{X_{t} \in\right.$ $F$ for some $t \in G\}>0$ for every $x$. (ii) If $X$ is $\mathbf{b}$-related for $G$ and $X^{b}$ is continuous in probability, then $\mathcal{E}(F)=\infty \Longrightarrow F$ is polar. (iii) If $X$ is $\mathbf{b}$-related for $G$ and $X^{b}$ has stationary increments and $K(y)>0$ for a.e. $y$, then for any $x$,

$$
P^{x}\left\{X_{t} \in F \text { for some } t \in G\right\}>0 \Longleftrightarrow \mathcal{E}_{b}^{1}(F)<\infty,
$$

where $\mathcal{E}_{b}^{1}(F)$ is given by (2.28).

Example 1. Let $X: \mathbb{R}^{N} \rightarrow \mathbb{R}^{d}$ be any rcll Markov field continuous in probability. Assume that $X$ has $\mathbf{b}$ and an a.e. positive $\phi$-potential density. Then for any $x, y \in \mathbb{R}^{d}, P^{x}\left\{X_{t}=y\right.$ for some $\left.t \in(0, \infty)^{N}\right\}>0$ iff $\int_{\mathbb{R}^{d}} Q_{\eta}^{X}(\xi) d \xi<\infty$ for some $\eta \in \mathcal{P}\left((0, \infty)^{N}\right)$. If in addition $X$ has stationary increments, then for any $x, y \in \mathbb{R}^{d}$, $P^{x}\left\{X_{t}=y\right.$ for some $\left.t \in(0, \infty)^{N}\right\}>0$ iff $\int_{\mathbb{R}^{d}} Q_{\kappa}^{X}(\xi) d \xi<\infty$.

Example 2. Let $X^{i}: \mathbb{R}^{N_{i}} \rightarrow \mathbb{R}^{d_{i}}, 1 \leq i \leq k$, be independent rcll Markov fields, each of which is continuous in probability, and has $\mathbf{b}_{i}$ and an a.e. positive $\phi_{i^{-}}$ potential density $K_{i}$. Define

$$
\tilde{X}_{u}=\left(X_{t_{1}}^{1}, \cdots, X_{t_{k}}^{k}\right), u=\left(t_{1}, \cdots, t_{k}\right), t_{i} \in \mathbb{R}^{N_{i}} .
$$

Since the $X^{i}$ are independent, $\widetilde{X}$ is a Markov field as well. It can be checked directly that $\widetilde{X}$ has a $\phi(u)=\phi_{1}\left(t_{1}\right) \cdots \phi_{k}\left(t_{k}\right)$-potential density $K(x)=K_{1}\left(x^{1}\right) \cdots K_{k}\left(x^{k}\right)$, where $u=\left(t_{1}, \cdots, t_{k}\right), t_{i} \in(0, \infty)^{N_{i}}, x=\left(x^{1}, \cdots, x^{k}\right), x^{i} \in \mathbb{R}^{d_{i}}$. Thus, $K$ too is a.e. positive. By Lemma 2.1 (check the field $Z^{2}$ ), $\widetilde{X}$ has b. Suppose that $X^{i}$ has the law of $X: \mathbb{R}^{N} \rightarrow \mathbb{R}^{d}$ for each $i$ and that $X$ has stationary increments. Let $F$ be any Borel subset of $\mathbb{R}^{d}$. By Theorem 3.2, a necessary and sufficient condition for $F$ to contain points of $\bigcap_{i=1}^{k} X^{i}\left((0, \infty)^{N}\right)$ with positive probability (under any $P^{x}$ ) is that there exists some $\mu \in \mathcal{P}(F)$ such that

$$
\int_{\mathbb{R}^{k d}}\left|\hat{\mu}\left(\xi_{1}+\cdots+\xi_{k}\right)\right|^{2} Q_{\kappa}^{X}\left(\xi_{1}\right) \cdots Q_{\kappa}^{X}\left(\xi_{k}\right) d \xi_{1} \cdots d \xi_{k}<\infty .
$$

If $F$ is compact and $F^{o} \neq \emptyset$, then in (3.2), $\mu=\lambda_{F}$, the normalized Lebesgue measure on $F$.

Example 3. Let $X^{i}: \mathbb{R}^{N_{i}} \rightarrow \mathbb{R}^{d}, 1 \leq i \leq k$, be independent rcll Markov fields, each of which is continuous in probability and has $\mathbf{b}_{i}$. Define $Y_{u}=X_{t_{1}}^{1}+\cdots+X_{t_{k}}^{k}, u=$ $\left(t_{1}, \cdots, t_{k}\right), t_{i} \in \mathbb{R}^{N_{i}}$. By independence, $Y$ is a Markov field. By Lemma 2.1 (cf. the field $Z^{4}$ ), $Y$ has b. Let $\zeta_{i}$ be an r.v. taking values in $\mathbb{R}_{+}^{N_{i}}$ with density function $\phi_{i}$ where $\phi_{i}(t)>0$ for a.e. $t \in(0, \infty)^{N_{i}}$. Assume that the $\zeta_{i}$ and $X^{i}$ are all independent. The distribution of $U=X_{\zeta_{1}}^{1}+\cdots+X_{\zeta_{k}}^{k}$ is given by $\nu=\nu_{1} \star \cdots \star \nu_{k}$, where $\nu_{i}$ is the distribution of $X_{\zeta_{i}}^{i}$. Thus, if $X_{\zeta_{1}}^{1}$ has a density, i.e., $X^{1}$ has a $\phi_{1}$-potential density, then $U$ has a density, i.e., $Y$ has a $\phi(u)=\phi_{1}\left(t_{1}\right) \cdots \phi_{k}\left(t_{k}\right)$ potential density, where $u=\left(t_{1}, \cdots, t_{k}\right), t_{i} \in(0, \infty)^{N_{i}}$ and $\phi_{2}, \cdots, \phi_{k}$ are arbitrary. Assume that $X^{1}$ has a $\phi_{1}$-potential density and all $X^{i}$ have stationary increments. 
By Theorem 3.2, if $Q_{\kappa_{1}}^{X_{1}^{1}}(\xi) \cdots Q_{\kappa_{k}}^{X^{k}}(\xi) \notin L^{1}\left(\mathbb{R}^{d}\right)$, then $Y$ does not hit points, i.e., for any $x$ and $y$,

$$
P^{x}\left(X_{t_{1}}^{1}+\cdots+X_{t_{k}}^{k}=y \text { for some } t_{i} \in(0, \infty)^{N_{i}}, 1 \leq i \leq k\right)=0 .
$$

Example 4. Let $X^{i}: \mathbb{R}^{N_{i}} \rightarrow \mathbb{R}^{d}, 1 \leq i \leq k$, be independent rcll Markov fields, each of which is continuous in probability and has $\mathbf{b}_{i}$. Let $m=N_{1}+\cdots+N_{k}$. Define

$$
{ }^{k} \Lambda_{u}=\left(X_{t_{2}}^{2}-X_{t_{1}}^{1}, \cdots, X_{t_{k}}^{k}-X_{t_{k-1}}^{k-1}\right), u=\left(t_{1}, \cdots, t_{k}\right), t_{i} \in \mathbb{R}^{N_{i}}, 1 \leq i \leq k .
$$

Let us first show that ${ }^{k} \Lambda$ has b. First assume that $X_{0}^{1}=0$ a.s. and consider

$$
A=\left\{\left(\omega_{2}\left(t_{2}\right)-\omega_{1}\left(t_{1}\right), \cdots, \omega_{k}\left(t_{k}\right)-\omega_{k-1}\left(t_{k-1}\right)\right), \omega_{i} \in \Omega^{i}, \omega_{1}(0)=0\right\},
$$

where $\Omega^{i}$ is the sample space for $X^{i}$. Then $P(A)=1$. If

$$
\left(\omega_{2}\left(t_{2}\right)-\omega_{1}\left(t_{1}\right), \cdots\right)=\left(\omega_{2}^{\prime}\left(t_{2}\right)-\omega_{1}^{\prime}\left(t_{1}\right), \cdots\right), \forall t_{i} \in \mathbb{R}^{N_{i}},
$$

then $\omega_{2}^{\prime}\left(t_{2}\right)-\omega_{1}^{\prime}\left(t_{1}\right)=\omega_{2}\left(t_{2}\right)-\omega_{1}\left(t_{1}\right)$, which implies that $\omega_{2}^{\prime}\left(t_{2}\right)=\omega_{2}\left(t_{2}\right), \forall t_{2} \in$ $\mathbb{R}^{N_{2}}$, i.e., $\omega_{2}^{\prime}=\omega_{2}$ since $\omega_{1}^{\prime}(0)=\omega_{1}(0)=0$. Subsequently, $\omega_{1}^{\prime}=\omega_{1}$ and $\omega_{3}^{\prime}=\omega_{3} \Longrightarrow$ $\omega_{4}^{\prime}=\omega_{4} \Longrightarrow \cdots \Longrightarrow \omega_{k}^{\prime}=\omega_{k}$. Therefore, for $\theta(t)=\left(\omega_{2}\left(t_{2}\right)-\omega_{1}\left(t_{1}\right), \cdots, \omega_{k}\left(t_{k}\right)-\right.$ $\left.\omega_{k-1}\left(t_{k-1}\right)\right) \in A$,

$\overline{\mathbf{b}}(\theta)(t)=\left(\mathbf{b}_{2}\left(\omega_{2}\right)\left(t_{2}\right)-\mathbf{b}_{1}\left(\omega_{1}\right)\left(t_{1}\right), \cdots, \mathbf{b}_{k}\left(\omega_{k}\right)\left(t_{k}\right)-\mathbf{b}_{k-1}\left(\omega_{k-1}\right)\left(t_{k-1}\right)\right), t_{i} \in \mathbb{R}_{+}^{N_{i}}$, where $\mathbf{b}_{i}$ is independent of $\left(X^{i}\right)^{l}: X_{t}^{i}, t \in[0, l)^{N_{i}}$, is well defined. Since the $X^{i}$ are independent, $\overline{\mathbf{b}}$ is independent of $\left({ }^{k} \Lambda\right)^{l}$, which is the field ${ }^{k} \Lambda_{u}, u \in[0, l)^{m}$. Let

$$
A^{\prime}=\left\{\left(\omega_{2}\left(t_{2}\right)-\omega_{1}\left(t_{1}\right), \cdots, \omega_{k}\left(t_{k}\right)-\omega_{k-1}\left(t_{k-1}\right)\right), \omega_{i} \in\left(\Omega^{i}\right)^{\prime}, \omega_{1}(0)=0\right\} .
$$

We see that $A^{\prime} \subset A$ and $r\left(A^{\prime}\right)=r(A)$ because $r\left[\left(\Omega^{i}\right)^{\prime}\right]=\Omega_{l}^{i}$. For each $\theta \in A^{\prime}$, we have

$$
\begin{aligned}
& r(\overline{\mathbf{b}}(\theta))=\left(r\left(\mathbf{b}_{2}\left(\omega_{2}\right)\right)-r\left(\mathbf{b}_{1}\left(\omega_{1}\right)\right), \cdots, r\left(\mathbf{b}_{k}\left(\omega_{k}\right)\right)-r\left(\mathbf{b}_{k-1}\left(\omega_{k-1}\right)\right)\right) \\
& =\left(r\left(\omega_{2}\right)-r\left(\omega_{1}\right), \cdots, r\left(\omega_{k}\right)-r\left(\omega_{k-1}\right)\right)=r(\theta) .
\end{aligned}
$$

Thus, ${ }^{k} \Lambda$ has b. We next try to dump the assumption that $X_{0}^{1}=0$ a.s. In that case it is still possible to use the idea of introducing the equivalence classes to define $\overline{\mathbf{b}}$ as in the proof of Lemma 2.1. We leave the rest to the reader. The proof that ${ }^{k} \Lambda$ has $\mathbf{b}$ is complete.

Now write

$$
\begin{aligned}
& { }^{k} \Lambda_{u}=\left(-X_{t_{1}}^{1}, 0, \cdots, 0\right)+\left(X_{t_{2}}^{2},-X_{t_{2}}^{2}, 0, \cdots, 0\right) \\
& +\left(0, X_{t_{3}}^{3},-X_{t_{3}}^{3}, 0, \cdots, 0\right)+\left(0, \cdots, 0, X_{t_{k-1}}^{k-1},-X_{t_{k-1}}^{k-1}\right)+\left(0, \cdots, 0, X_{t_{k}}^{k}\right) .
\end{aligned}
$$

The field $\left(0, \cdots, 0, X_{t_{i}}^{i},-X_{t_{i}}^{i}, 0, \cdots, 0\right)$ is Markov because the Markov property is defined in terms of law, i.e., all joint distributions. ${ }^{k} \Lambda$ is a sum of independent Markov fields. It follows that ${ }^{k} \Lambda$ is Markov. Consider the random variable

$$
V=\left(X_{\zeta_{2}}^{2}-X_{\zeta_{1}}^{1}, X_{\zeta_{3}}^{3}-X_{\zeta_{2}}^{2}, \cdots, X_{\zeta_{k}}^{k}-X_{\zeta_{k-1}}^{k-1}\right)
$$

where the $\zeta_{i}$ are as given in Example 3. The linear transformation pattern above shows that if the $k$ independent r.v.s $X_{\xi_{i}}^{i}$ contain $k-1$ members which have densities, then $V$ has a density, i.e., ${ }^{k} \Lambda$ has a $\phi(u)=\phi_{1}\left(t_{1}\right) \cdots \phi_{k}\left(t_{k}\right)$-potential density, where $u=\left(t_{1}, \cdots, t_{k}\right), t_{i} \in(0, \infty)^{N_{i}}$. Furthermore, calculations show that if the marginal densities are all a.e. positive, then the resulting density of $V$ is also a.e. positive. 
Assume that the $X^{i}$ have $k-1$ of them having a.e. positive $\phi$-potential densities. By Theorem 3.2, for any $x$,

$$
\begin{aligned}
P^{x}\left\{\bigcap_{i=1}^{k} X^{i}\left((0, \infty)^{N_{i}}\right) \neq \emptyset\right\}>0 \\
\quad \Longleftrightarrow \int_{\mathbb{R}^{d(k-1)}} Q_{\mu}^{k} \Lambda(\xi) d \xi<\infty \text { for some } \mu \in \mathcal{P}\left((0, \infty)^{m}\right)
\end{aligned}
$$

If each $X^{i}$ has stationary increments in addition, then

$$
P^{x}\left\{\bigcap_{i=1}^{k} X^{i}\left((0, \infty)^{N_{i}}\right) \neq \emptyset\right\}>0 \Longleftrightarrow \int_{\mathbb{R}^{d(k-1)}} Q_{\kappa}^{k} \Lambda_{(\xi)} d \xi<\infty .
$$

$\left[X_{t}^{i}-X_{s}^{i} \stackrel{d}{=} X_{t-s}^{i} \Longrightarrow\left(X_{t}^{i}-X_{s}^{i},-\left(X_{t}^{i}-X_{s}^{i}\right)\right) \stackrel{d}{=}\left(X_{t-s}^{i},-X_{t-s}^{i}\right) \Longrightarrow\right.$ the field $\left(X_{t}^{i},-X_{t}^{i}\right)$ has stationary increments. Since the fields $X^{i}$ are independent, ${ }^{k} \Lambda$ has stationary increments.]

Example 5. Let $X: \mathbb{R}^{N} \rightarrow \mathbb{R}^{d}$ be an rcll Markov field, where $X$ is Markov at every $t \in \mathbb{R}_{+}^{N}$. Let $\sigma: \mathbb{R}_{+}^{n} \rightarrow \mathbb{R}_{+}^{N}$ be an rcll Markov field independent of $X$, where $\sigma$ is Markov at every $s \in \mathbb{R}_{+}^{n}$. Assume that $\sigma$ is increasing, i.e., $0 \preceq s_{1} \preceq s_{2} \Longrightarrow \preceq$ $\sigma_{s_{1}} \preceq \sigma_{s_{2}}$. Consider the very important composite field $X \circ \sigma$. Let $t_{1}, t_{2}, \cdots, t_{p}$ be points in $(0, \infty)^{N}$. Let $s \in \mathbb{R}_{+}^{n}$. We have

$$
\begin{aligned}
& P\left[X\left(\sigma_{t_{1}+s}\right)-X\left(\sigma_{s}\right) \in \cdot, \cdots, X\left(\sigma_{t_{p}+s}\right)-X\left(\sigma_{s}\right) \in \cdot, X\left(\sigma_{s}\right) \in \cdot\right] \\
& =\int_{\left\{0 \preceq y_{i}, 1 \leq i \leq p\right\}} P\left[X\left(y_{1}+x\right)-X(x) \in \cdot, \cdots, X\left(y_{p}+x\right)-X(x) \in \cdot, X(x) \in \cdot\right] \\
& \cdot P\left(\sigma_{t_{1}+s}-\sigma_{s} \in d y_{1}, \cdots, \sigma_{t_{p}+s}-\sigma_{s} \in d y_{p}, \sigma_{s} \in d x\right) \\
& \text { (since } \left.X \text { and } \sigma \text { are independent and since } 0 \preceq \sigma_{t_{i}+s}-\sigma_{s}\right) \\
& =\int_{\left\{0 \preceq y_{i}, 1 \leq i \leq p\right\}} P\left[X\left(y_{1}\right) \in \cdot, \cdots, X\left(y_{p}\right) \in \cdot\right] P[X(x) \in \cdot] \\
& \cdot P\left(\sigma_{t_{1}+s}-\sigma_{s} \in d y_{1}, \cdots, \sigma_{t_{p}+s}-\sigma_{s} \in d y_{p}, \sigma_{s} \in d x\right) \text { (since } X \text { is Markov) } \\
& =\int_{\left\{0 \preceq y_{i}, 1 \leq i \leq p\right\}} P\left[X\left(y_{1}\right) \in \cdot, \cdots, X\left(y_{p}\right) \in \cdot\right] P[X(x) \in \cdot] \\
& \cdot P\left(\sigma_{t_{1}} \in d y_{1}, \cdots, \sigma_{t_{p}} \in d y_{p}\right) P\left(\sigma_{s} \in d x\right)(\text { since } \sigma \text { is Markov) } \\
& =\int P\left[X\left(y_{1}\right) \in \cdot, \cdots, X\left(y_{p}\right) \in \cdot\right] P\left(\sigma_{t_{1}} \in d y_{1}, \cdots, \sigma_{t_{p}} \in d y_{p}\right) \\
& \cdot \int P(X(x) \in \cdot) P\left(\sigma_{s} \in d x\right) \\
& =P\left[X\left(\sigma_{t_{1}}\right) \in \cdot, \cdots, X\left(\sigma_{t_{p}}\right) \in \cdot\right] P\left[X\left(\sigma_{s}\right) \in \cdot\right] .
\end{aligned}
$$

We have shown that $X \circ \sigma$ is a Markov field. It is quite related to argue that $X \circ \sigma$ has $\mathbf{b}$ if both $X$ and $\sigma$ have $\mathbf{b}$. But some steep hurdles remain. One can show that if a symmetric Markov field $X$ has an integral representation and is an $M$-field, then $X \circ \sigma$ is b-related. See the proof of Proposition 4.5 below for a similar argument. Assume that $X$ and $\sigma$ are both Lévy fields. (This type of Markov field is defined in the next section. Additive Lévy processes for example are Lévy fields.) Then $X \circ \sigma$ is also a Lévy field and hence has $\mathbf{b}$ on $\mathbb{R}_{+}^{N}$ but does not have $\mathbf{b}$ on $\mathbb{R}^{N}$ in general. 


\section{TyPes OF FIELDS HAVING $\mathbf{b}$}

Proposition 2.2 can be improved when one of the fields has stationary increments.

Proposition 4.1. (i) Let $X: \mathbb{R}^{N} \rightarrow \mathbb{R}^{d}$ be an rcll field having stationary increments and $\mathbf{b}$. Let $\mathbb{R}_{i}^{N}$ be the ith closed quadrant $\left(\mathbb{R}_{1}^{N}=\mathbb{R}_{+}^{N}\right)$ and let $\kappa^{i}(d t)=$ $e^{-\sum_{j=1}^{N}\left|t_{j}\right|} d t, t=\left(t_{1}, \cdots, t_{N}\right) \in \mathbb{R}_{i}^{N}$, vanishing elsewhere, be a probability measure on $\mathbb{R}_{i}^{N}\left(\kappa^{1}=\kappa\right)$. Then for any Borel set $F \subset \mathbb{R}^{d}$ and each $i$,

$$
E\left\{\lambda_{d}\left(X\left(\mathbb{R}_{i}^{N}\right)-F\right)\right\}>0 \Longrightarrow \int_{\mathbb{R}^{d}}|\hat{\mu}(\xi)|^{2} Q_{\kappa^{i}}^{X}(\xi) d \xi<\infty \text { for some } \mu \in \mathcal{P}(F) .
$$

(ii) Let $Y: \mathbb{R}^{n} \rightarrow \mathbb{R}^{d}$ be an rcll field continuous in probability having $\mathbf{b}$. Then for any Borel set $G \subset \mathbb{R}^{n}$,

$$
E\left\{\lambda_{d}(Y(G))\right\}>0 \Longrightarrow \int_{\mathbb{R}^{d}} Q_{\eta}^{Y}(\xi) d \xi<\infty \text { for some } \eta \in \mathcal{P}(G)
$$

(iii) (i) and (ii) are just special cases of the general result

$$
E\left\{\lambda_{d}\left[X\left(\mathbb{R}_{i}^{N}\right) \pm Y(G)\right]\right\}>0 \Longrightarrow \int_{\mathbb{R}^{d}} Q_{\eta}^{Y}(\xi) Q_{\kappa^{i}}^{X}(\xi) d \xi<\infty \text { for some } \eta \in \mathcal{P}(G),
$$

where $X$ and $Y$ are independent.

In (i) and (iii), if $X$ has $\mathbf{b}$ on $\mathbb{R}_{+}^{N}$, then the results still hold for $\mathbb{R}_{+}^{N}$ and $\kappa$, i.e.,

$$
E\left\{\lambda_{d}\left[X\left(\mathbb{R}_{+}^{N}\right) \pm Y(G)\right]\right\}>0 \Longrightarrow \int_{\mathbb{R}^{d}} Q_{\eta}^{Y}(\xi) Q_{\kappa}^{X}(\xi) d \xi<\infty \text { for some } \eta \in \mathcal{P}(G) \text {. }
$$

In (ii) and (iii), if $Y$ has $\mathbf{b}$ on $\mathbb{R}_{+}^{n}$, then $G \subset \mathbb{R}_{+}^{n}$ is assumed.

Proof. We begin by drawing some technical facts from harmonic analysis. Let $\mathbf{x}: \mathbb{R}^{N} \rightarrow \mathbb{R}^{d}$ be an rcll field. For $s, t \in \mathbb{R}^{N}$ and $f$ with $f, \hat{f} \in L^{1}$, we define

$$
P_{s, t} f(x)=E\left[f\left(\mathbf{x}_{t}-\mathbf{x}_{s}+x\right)\right], x \in \mathbb{R}^{d} .
$$

Using the inversion formula for convolution yields

$$
P_{s, t} f(x)=(2 \pi)^{-d} \int_{\mathbb{R}^{d}} e^{i x \cdot \xi} \hat{f}(-\xi) E e^{i \xi \cdot\left(\mathbf{x}_{t}-\mathbf{x}_{s}\right)} d \xi, x \in \mathbb{R}^{d} .
$$

Given any probability measure $\mu$ on $\mathbb{R}^{N}$ and $s \in \mathbb{R}^{N}$, we write the probability measure $\mu_{(s)}(\bullet)=\mu(\bullet+s)$ on $\mathbb{R}^{N}$ for the shift of $\mu$. Set $[0, l]_{i}^{N}=\mathbb{R}_{i}^{N} \cap[-l, l]^{N}, l \in$ $(0, \infty)$. If $\bar{b} \in[0, l]_{i}^{N}, l \in(0, \infty)$, then $\kappa_{(b-\bar{b})}^{i}(\bullet) \leq e^{l N} \kappa_{(b)}^{i}(\bullet)$ for all $b \in \mathbb{R}_{i}^{N}$. When $\bar{b} \in \mathbb{R}_{i}^{N}$, the shift is downward. Thus, the said inequality holds. It is also clear that if a field $\mathbf{x}: \mathbb{R}^{N} \rightarrow \mathbb{R}^{d}$ has stationary increments, then for all $s \in \mathbb{R}^{N}$, $Q_{\mu_{(s)}}^{\mathbf{x}}(\xi)=Q_{\mu}^{\mathbf{x}}(\xi)$.

Define $H(s, t, \xi)=E e^{i \xi \cdot\left(Y_{s}-Y_{t}\right)}: \mathbb{R}^{n} \times \mathbb{R}^{n} \times \mathbb{R}^{d} \rightarrow \mathbb{C}$. Since $Y$ is continuous in probability, $H$ is a bounded continuous function.

Let us assume that $Y$ has stationary increments (only for now) and $X$ and $Y$ are independent, and prove that

$$
E\left\{\lambda_{d}\left[X\left(\mathbb{R}_{i}^{N}\right) \pm Y(G)\right]\right\}>0 \Longrightarrow \int_{\mathbb{R}^{d}} Q_{\mu}^{Y}(\xi) Q_{\kappa^{i}}^{X}(\xi) d \xi<\infty \text { for some } \mu \in \mathcal{P}(G)
$$


Since $-X$ is adapted to $X,-X$ has $\mathbf{b}$ as well. Clearly $-X$ has stationary increments. Furthermore, $Q_{\kappa}^{-X}(\xi)=Q_{\kappa}^{X}(\xi)$ and $\lambda_{d}\left[X\left(\mathbb{R}_{+}^{N}\right)-Y(G)\right]=\lambda_{d}\left[-X\left(\mathbb{R}_{+}^{N}\right)+\right.$ $Y(G)$ ]. Thus, we only have to prove (4.4) in the "+" case.

Define $Z=Y+X: \mathbb{R}^{n+N} \rightarrow \mathbb{R}^{d}$ by $Z_{(t, s)}=Y_{t}+X_{s}, t \in \mathbb{R}^{n}, s \in \mathbb{R}^{N}$. Without loss of generality, we may assume that

$$
E\left\{\lambda_{d}\left(Z\left(G \times[0, l]_{i}^{N}\right) \cap B(0, R)\right)\right\}>0,
$$

where $G$ is a compact set, $l \in(0, \infty)$ and $R$ is a sufficiently large number. We will establish a precise inequality:

$$
\int_{\mathbb{R}^{d}} Q_{\mu}^{Y}(\xi) Q_{\kappa^{i}}^{X}(\xi) d \xi \leq \frac{2 e^{2 l N}(2 \pi)^{d}}{E\left\{\lambda_{d}\left(Z\left(G \times[0, l]_{i}^{N}\right) \cap B(0, R)\right)\right\}} \text {, for some } \mu \in \mathcal{P}(G) .
$$

Part I. Let $G^{\delta}$ be the closed $\delta$-enlargement of $G$ for $\delta>0$, that is, the smallest compact set such that for each point $s=\left(s_{1}, \cdots, s_{N}\right) \in G,\left[s_{1}, s_{1}+\delta\right] \times \cdots \times$ $\left[s_{N}, s_{N}+\delta\right] \subset G^{\delta}$. For $s, t \in \mathbb{R}^{n+N}$ and $f: \mathbb{R}^{d} \rightarrow \mathbb{R}_{+}$with $f, \hat{f} \in L^{1}$, we define

$$
P_{s, t} f(x)=E\left[f\left(Z_{t}-Z_{s}+x\right)\right], x \in \mathbb{R}^{d} .
$$

For $\varepsilon>0$, let $f^{\varepsilon}(x)=\left(2 \pi \varepsilon^{2}\right)^{-d / 2} e^{-\frac{|x|^{2}}{2 \varepsilon^{2}}}, x \in \mathbb{R}^{d}$. Note that $f^{\varepsilon}, \widehat{f^{\varepsilon}} \in L^{1} \cap L^{2}$. Let $a=(v, b), v \in \mathbb{R}^{n}, b \in \mathbb{R}_{i}^{N}$. Let $\nu^{\delta}=\mu^{\delta} \times \kappa^{i}$, where $\mu^{\delta}$ is some probability measure on $G^{\delta}$. Since $X$ and $Y$ are independent, since $\widehat{f \varepsilon}(-\xi)=e^{-\frac{\varepsilon^{2}}{2}|\xi|^{2}}$ and since we are allowed to interchange the order of integration owing to the integration factor $e^{-\frac{\varepsilon^{2}}{2}|\xi|^{2}}$, by $(4.3)$ we have, for any $a=(v, b)$,

$$
\begin{aligned}
& \int_{\mathbb{R}^{n+N}} P_{a, t} f^{\varepsilon}(0) \nu^{\delta}(d t) \\
& =(2 \pi)^{-d} \int_{\mathbb{R}^{n}} \int_{\mathbb{R}^{d}} e^{-\frac{\varepsilon^{2}}{2}|\xi|^{2}} E e^{i \xi \cdot\left(Y_{u}-Y_{v}\right)} \int_{\mathbb{R}_{i}^{N}} E e^{i \xi \cdot\left(X_{b^{\prime}}-X_{b}\right)} \kappa^{i}\left(d b^{\prime}\right) d \xi \mu^{\delta}(d u) .
\end{aligned}
$$

Integrating $\kappa^{i}(d b)$ over $\mathbb{R}_{i}^{N}$ and interchanging the order of integration yields

$$
\begin{aligned}
& \int_{\mathbb{R}_{i}^{N}}\left(\int_{\mathbb{R}^{n+N}} P_{a, t} f^{\varepsilon}(0) \nu^{\delta}(d t)\right) \kappa^{i}(d b) \\
& =(2 \pi)^{-d} \int_{\mathbb{R}^{n}} \int_{\mathbb{R}^{d}} e^{-\frac{\varepsilon^{2}}{2}|\xi|^{2}} E e^{i \xi \cdot\left(Y_{u}-Y_{v}\right)} Q_{\kappa^{i}}^{X}(\xi) d \xi \mu^{\delta}(d u) .
\end{aligned}
$$

It is abundantly clear that $f^{\varepsilon}$ is a Lipschitz continuous function. Let $D(\varepsilon)$ be the Lipschitz constant of $f^{\varepsilon}$. By the definition of $P_{s, t} f^{\varepsilon}$, we have

$$
D(\varepsilon) \delta+\inf _{|z| \leq \delta} P_{a, t} f^{\varepsilon}(z) \geq P_{a, t} f^{\varepsilon}(0) .
$$

Since both $\nu^{\delta}$ and $\kappa^{i}$ are probability measures, we have

$$
\begin{gathered}
D(\varepsilon) \delta+\int_{\mathbb{R}_{i}^{N}}\left(\int_{\mathbb{R}^{n+N}} \inf _{|z| \leq \delta} P_{a, t} f^{\varepsilon}(z) \nu^{\delta}(d t)\right) \kappa^{i}(d b) \\
\geq \int_{\mathbb{R}_{i}^{N}}\left(\int_{\mathbb{R}^{n+N}} P_{a, t} f^{\varepsilon}(0) \nu^{\delta}(d t)\right) \kappa^{i}(d b) .
\end{gathered}
$$


Let $x$ be any vector in $\mathbb{R}^{d}$ such that $|x| \leq \delta$. Then $P_{a, t} f^{\varepsilon}(x) \geq \inf _{|z| \leq \delta} P_{a, t} f^{\varepsilon}(z)$. It follows that

$$
\begin{aligned}
& \int_{\mathbb{R}_{i}^{N}}\left(\int_{\mathbb{R}^{n+N}} P_{a, t} f^{\varepsilon}(x) \nu^{\delta}(d t)\right) \kappa^{i}(d b) \\
& \geq(2 \pi)^{-d} \int_{\mathbb{R}^{n}} \int_{\mathbb{R}^{d}} e^{-\frac{\varepsilon^{2}}{2}|\xi|^{2}} H(u, v, \xi) Q_{\kappa^{i}}^{X}(\xi) d \xi \mu^{\delta}(d u)-D(\varepsilon) \delta .
\end{aligned}
$$

Let $\alpha=(v, \bar{b})$, where $\bar{b} \in \mathbb{R}_{i}^{N}$. Since $X$ and $Y$ are independent and since $\left(s_{2}, t_{2}\right)-$ $\left(s_{1}, t_{1}\right)=\left(s_{2}-s_{1}, t_{2}-t_{1}\right)$, where $s_{i} \in \mathbb{R}^{n}$ and $t_{i} \in \mathbb{R}^{N}, Z$ has stationary increments on $\mathbb{R}^{n+N}$. It follows that

$$
P_{a, t} f^{\varepsilon}=P_{\alpha, t-a+\alpha} f^{\varepsilon}=P_{0, t-a} f^{\varepsilon} .
$$

Therefore, for all $x \in \mathbb{R}^{d}$,

$$
P_{a, t} f^{\varepsilon}(x)=P_{\alpha, t-a+\alpha} f^{\varepsilon}(x) .
$$

We will denote $\nu_{(a-\alpha)}^{\delta}$ simply by $\nu_{(b-\bar{b})}^{\delta}$ because $v-v=0$. Let $w=t-a+\alpha$. We have for all $x \in \mathbb{R}^{d}$,

$$
\begin{aligned}
& \int_{\mathbb{R}^{n+N}} P_{a, t} f^{\varepsilon}(x) \nu^{\delta}(d t) \\
& =\int_{\mathbb{R}^{n+N}} P_{\alpha, t-a+\alpha} f^{\varepsilon}(x) \nu^{\delta}(d t) \\
& =\int_{\mathbb{R}^{n+N}} P_{\alpha, w} f^{\varepsilon}(x) \nu_{(b-\bar{b})}^{\delta}(d w) .
\end{aligned}
$$

Recall that if $\bar{b} \in[0, l]_{i}^{N}$, then $\nu_{(b-\bar{b})}^{\delta}(\bullet) \leq e^{l N} \nu_{(b)}^{\delta}(\bullet)$. Therefore, for all $\bar{b} \in[0, l]_{i}^{N}$ and all $x \in \mathbb{R}^{d}$,

$$
\int_{\mathbb{R}_{i}^{N}}\left(\int_{\mathbb{R}^{n+N}} P_{a, t} f^{\varepsilon}(x) \nu^{\delta}(d t)\right) \kappa^{i}(d b) \leq e^{l N} \int_{\mathbb{R}_{i}^{N}}\left(\int_{\mathbb{R}^{n+N}} P_{\alpha, t} f^{\varepsilon}(x) \nu_{(b)}^{\delta}(d t)\right) \kappa^{i}(d b) .
$$

(4.7) and (4.8) imply that for all $\alpha=(v, \bar{b})$ where $\bar{b} \in[0, l]_{i}^{N}, v \in \mathbb{R}^{n}$, and all $x \in \mathbb{R}^{d}$ with $|x| \leq \delta$,

$e^{l N} \int_{\mathbb{R}_{i}^{N}} N_{b}(\alpha, x) \kappa^{i}(d b) \geq(2 \pi)^{-d} \int_{\mathbb{R}^{n}} \int_{\mathbb{R}^{d}} e^{-\frac{\varepsilon^{2}}{2}|\xi|^{2}} H(u, v, \xi) Q_{\kappa^{i}}^{X}(\xi) d \xi \mu^{\delta}(d u)-D(\varepsilon) \delta$, where

$$
N_{b}(\alpha, x)=\int_{\mathbb{R}^{n+N}} P_{\alpha, t} f^{\varepsilon}(x) \nu_{(b)}^{\delta}(d t)
$$

Part II. In the following we will use the space $(\Omega, \mathcal{F})$ of rcll functions from $\mathbb{R}^{n+N}$ into $\mathbb{R}^{d}$ endowed with the canonical Skorohod topology. Let $P$ be the complete probability measure on $\Omega$ given by $Z$, i.e., the law of $Z$, where we assume that $Z$ is in the canonical form. Let $P^{x}, x \in \mathbb{R}^{d}$, be the probability measure on $(\Omega, \mathcal{F})$ given by the path spatial shift $P^{x}\{\bullet\}=P^{0}\{x+\bullet\}$, where $x+A=\{x+\omega: \omega \in A\}$, $A \in \mathcal{F}, P^{0}=P$, and $x+\omega \in \Omega$ is defined by $(x+\omega)(t)=x+\omega(t), t \in \mathbb{R}^{N}$. This gives rise to a $\sigma$-finite measure $P_{\lambda}$ on $(\Omega, \mathcal{F})$ given by $P_{\lambda}(\bullet)=\int_{\mathbb{R}^{d}} P^{x}(\bullet) d x$.

By Lemma 2.1 (cf. the field $\left.Z^{4}\right), Z$ has b. The domain $J\left(l_{1}\right)$ for $Z^{l_{1}}$ can be $\left(\left[-l_{1}, l_{1}\right) \times \mathbb{R}^{n-1}\right) \times\left(\left[-l_{1}, l_{1}\right) \times \mathbb{R}^{N-1}\right)$, or $\left[-l_{1}, l_{1}\right) \times \mathbb{R}^{n+N-1}$, or $\left[-l_{1}, l_{1}\right)^{n+N}$. In any case, we fix a large $l_{1}$ satisfying $G^{\delta} \times[0, l]_{i}^{N} \subset J\left(l_{1}\right)$. Let $\mathbf{b}: \Omega \rightarrow \Omega$ be the 
measurable mapping such that (1) $\mathbf{b}$ is independent of $Z^{l_{1}}$ and (2) for some event $\Omega^{\prime} \subset \Omega$ with $r\left(\Omega^{\prime}\right)=\Omega_{l_{1}}, r\left[\mathbf{b}\left(\omega^{\prime}\right)\right]=r\left(\omega^{\prime}\right)$ for each $\omega^{\prime} \in \Omega^{\prime}$, where $\Omega_{l_{1}}$ is the space of rcll functions from $J\left(l_{1}\right)$ into $\mathbb{R}^{d}$, the sample space of $Z^{l_{1}}$, and $r: \Omega \rightarrow \Omega_{l_{1}}$ is the restriction mapping. Let the probability measure $P_{l_{1}}$ be the natural restriction of $P$ to $\Omega_{l_{1}}$, i.e., the law of $Z^{l_{1}}$. We then define the $\sigma$-finite measure $\left[P_{l_{1}}\right]_{\lambda}$ on $\Omega_{l_{1}}$ in an analogous way. Consider the event

$$
A^{\delta}=\left\{\omega \in \Omega: \omega(t) \in B(0, \delta) \text { for some } t \in G^{\delta} \times[0, l]_{i}^{N}, \omega(0) \in B(0, R)\right\} .
$$

The fact that $\omega$ is right continuous and $G^{\delta}$ is the closed $\delta$-enlargement of the compact set $G$ implies that

$$
A^{\delta}=\left\{\omega \in \Omega: \omega(t) \in B(0, \delta) \text { for some } t \in\left(G^{\delta} \times[0, l]_{i}^{N}\right) \cap \mathbb{Q}^{n+N}, \omega(0) \in B(0, R)\right\} .
$$

(This also shows that $A^{\delta} \in \mathcal{F}$.) Note that $Z$ and $Z^{l_{1}}$ are both in the canonical form. By Fubini's theorem and the fact that $-B(0, r)=B(0, r)$,

$$
P_{\lambda}\left\{A^{\delta}\right\}=E\left\{\lambda_{d}\left(\left[Z\left(G^{\delta} \times[0, l]_{i}^{N}\right)+B(0, \delta)\right] \cap B(0, R)\right)\right\},
$$

which implies that

$$
\infty>\lambda_{d}[B(0, R)] \geq P_{\lambda}\left\{A^{\delta}\right\} \geq E\left\{\lambda_{d}\left(Z\left(G \times[0, l]_{i}^{N}\right) \cap B(0, R)\right)\right\}>0 .
$$

Clearly,

$$
r\left(A^{\delta}\right)=\left\{\omega \in \Omega_{l_{1}}: \omega(t) \in B(0, \delta) \text { for some } t \in G^{\delta} \times[0, l]_{i}^{N}, \omega(0) \in B(0, R)\right\} .
$$

Since $r\left(\Omega^{\prime}\right)=\Omega_{l_{1}}, r\left(\Omega^{\prime} \cap A^{\delta}\right)=r\left(A^{\delta}\right)$. Let $S \subset A^{\delta}$ be any event. Recall that $G^{\delta} \times[0, l]_{i}^{N} \subset J\left(l_{1}\right)$. Let $E_{l_{1}}$ be the expectation for $P_{l_{1}}$. We have

$$
\begin{gathered}
P_{\lambda}(S)=E\left\{\lambda_{d}\left(\left[Z\left(G^{\delta} \times[0, l]_{i}^{N}\right)+B(0, \delta)\right] \cap B(0, R)\right) 1_{S}\right\} \\
=E_{l_{1}}\left\{\lambda_{d}\left(\left[Z^{l_{1}}\left(G^{\delta} \times[0, l]_{i}^{N}\right)+B(0, \delta)\right] \cap B(0, R)\right) 1_{r(S)}\right\}=\left[P_{l_{1}}\right]_{\lambda}(r(S)) .
\end{gathered}
$$

In particular, $P_{\lambda}\left\{A^{\delta}\right\}=\left[P_{l_{1}}\right]_{\lambda}\left\{r\left(A^{\delta}\right)\right\}$. Set $\widetilde{A}^{\delta}=\Omega^{\prime} \cap A^{\delta}$. Since $r\left(\widetilde{A}^{\delta}\right)=r\left(A^{\delta}\right)$,

$$
P_{\lambda}\left\{\widetilde{A}^{\delta}\right\}=\left[P_{l_{1}}\right]_{\lambda}\left\{r\left(\widetilde{A}^{\delta}\right)\right\}=\left[P_{l_{1}}\right]_{\lambda}\left\{r\left(A^{\delta}\right)\right\}=P_{\lambda}\left\{A^{\delta}\right\} .
$$

For each $\omega \in A^{\delta}$, denote by $\zeta_{\omega}^{\delta}$ the set of the points $u \in\left[G^{\delta} \cap \mathbb{Q}^{n}\right] \times\left[[0, l]_{i}^{N} \cap \mathbb{Q}^{N}\right]$ such that $|\omega(u)|<\delta$. Clearly, $\zeta_{\omega}^{\delta}$ is a countably infinite set, and the set $\zeta_{\omega}^{\delta}$ remains the same for both $\omega \in A^{\delta}$ and $\omega \in r\left(A^{\delta}\right)$. Note that the set $r\left(A^{\delta}\right) \subset \Omega_{l_{1}}$ is measuable. Thus, using the Skorohod topology on $\Omega_{l_{1}}$, we are able to define a Borel mapping $\theta: \Omega_{l_{1}} \rightarrow\left[G^{\delta} \cap \mathbb{Q}^{n}\right] \times\left[[0, l]_{i}^{N} \cap \mathbb{Q}^{N}\right]$ such that for each $\omega \in r\left(A^{\delta}\right)$, $\theta(\omega) \in \zeta_{\omega}^{\delta}$, where the countable set $\left[G^{\delta} \cap \mathbb{Q}^{n}\right] \times\left[[0, l]_{i}^{N} \cap \mathbb{Q}^{N}\right]$ has its discrete topology. The restriction mapping $r$ from $\Omega$ to $\Omega_{l_{1}}$ is clearly measurable. Thus, $\phi=\theta \circ r: \Omega \rightarrow\left[G^{\delta} \cap \mathbb{Q}^{n}\right] \times\left[[0, l]_{i}^{N} \cap \mathbb{Q}^{N}\right]$ is a measurable mapping. Finally, for each $\omega^{\prime} \in \widetilde{A}^{\delta}$,

$$
\phi\left(\mathbf{b}\left(\omega^{\prime}\right)\right)=\theta\left(r\left(\mathbf{b}\left(\omega^{\prime}\right)\right)\right)=\theta\left(r\left(\omega^{\prime}\right)\right) \in \zeta_{r\left(\omega^{\prime}\right)}^{\delta}=\zeta_{\omega^{\prime}}^{\delta},
$$

since $r\left(\omega^{\prime}\right) \in r\left(A^{\delta}\right)$. Define

$$
\Theta^{\delta}=\phi \circ \mathbf{b}
$$

It is immediate that $\Theta^{\delta}$ is a random variable defined on $\Omega$ taking values in the countable set $\left[G^{\delta} \cap \mathbb{Q}^{n}\right] \times\left[[0, l]_{i}^{N} \cap \mathbb{Q}^{N}\right]$ with the properties that $\Theta^{\delta}$ is independent 
of $Z^{l_{1}}$ and $\Theta^{\delta}(\omega) \in \zeta_{\omega}^{\delta}, \forall \omega \in \widetilde{A}^{\delta}$. Let $\Theta^{\delta}=\left(T^{\delta}, \mathbf{t}^{\delta}\right)$, where $T^{\delta}: \Omega \rightarrow G^{\delta}, \mathbf{t}^{\delta}: \Omega \rightarrow$ $[0, l]_{i}^{N}$, and define

$$
\mu^{\delta}(\bullet)=\frac{P_{\lambda}\left\{T^{\delta} \in \bullet, \widetilde{A}^{\delta}\right\}}{P_{\lambda}\left\{\widetilde{A}^{\delta}\right\}},
$$

which is a probability measure on $G^{\delta}$, since $P_{\lambda}\left\{\widetilde{A}^{\delta}\right\}=P_{\lambda}\left\{A^{\delta}\right\} \in(0, \infty)$.

Part III. $\mu^{\delta}$ will be the one given by (4.16) from this point on. Recall that $Z$ is in the canonical form, i.e., $Z_{u}(\omega)=\omega(u)$ for each $\omega \in \Omega$ and each $u \in \mathbb{R}^{n+N}$. By the definition of $\Theta^{\delta}$,

$$
\left|Z_{\Theta^{\delta}(\omega)}(\omega)\right|<\delta \text { for each } \omega \in \widetilde{A}^{\delta} .
$$

Replacing $\alpha$ by $\Theta^{\delta}, x$ by $Z_{\Theta^{\delta}}$ and $v$ by $T^{\delta}$ in (4.9) yields

$$
\begin{aligned}
1_{\widetilde{A}^{\delta}} e^{l N} \int_{\mathbb{R}_{i}^{N}} N_{b}\left(\Theta^{\delta}, Z_{\Theta^{\delta}}\right) \kappa^{i}(d b) \\
\quad \geq\left\{(2 \pi)^{-d} \int_{\mathbb{R}^{n}} \int_{\mathbb{R}^{d}} e^{-\frac{\varepsilon^{2}}{2}|\xi|^{2}} H\left(u, T^{\delta}, \xi\right) Q_{\kappa^{i}}^{X}(\xi) d \xi \mu^{\delta}(d u)-D(\varepsilon) \delta\right\} 1_{\widetilde{A}^{\delta}},
\end{aligned}
$$

which implies that

$$
\begin{aligned}
1_{\widetilde{A}^{\delta}} D(\varepsilon) \delta+e^{l N} \int_{\mathbb{R}_{i}^{N}} N_{b}\left(\Theta^{\delta}, Z_{\Theta^{\delta}}\right) \kappa^{i}(d b) \\
\quad \geq 1_{\widetilde{A}^{\delta}}(2 \pi)^{-d} \int_{\mathbb{R}^{n}} \int_{\mathbb{R}^{d}} e^{-\frac{\varepsilon^{2}}{2}|\xi|^{2}} H\left(u, T^{\delta}, \xi\right) Q_{\kappa^{i}}^{X}(\xi) d \xi \mu^{\delta}(d u) .
\end{aligned}
$$

Applying the inequality $(a+b)^{2} \leq 2 a^{2}+2 b^{2}$ and Jensen's inequality, since $\kappa^{i}$ is a probability measure, (4.19) yields

$$
\begin{gathered}
1_{\widetilde{A}^{\delta}} 2(D(\varepsilon) \delta)^{2}+2 e^{2 l N} \int_{\mathbb{R}_{i}^{N}}\left[N_{b}\left(\Theta^{\delta}, Z_{\Theta^{\delta}}\right)\right]^{2} \kappa^{i}(d b) \\
\geq 1_{\widetilde{A}^{\delta}}(2 \pi)^{-2 d}\left\{\int_{\mathbb{R}^{n}} \int_{\mathbb{R}^{d}} e^{-\frac{\varepsilon^{2}}{2}|\xi|^{2}} H\left(u, T^{\delta}, \xi\right) Q_{\kappa^{i}}^{X}(\xi) d \xi \mu^{\delta}(d u)\right\}^{2} .
\end{gathered}
$$

Let $E_{\lambda}$ denote the expectation operator with respect to $P_{\lambda}$ for real-valued random variables $\mathbf{w}$ defined on $\Omega$. One can check that $E_{\lambda}(\mathbf{w})=\int_{\mathbb{R}^{d}} E^{x}(\mathbf{w}) d x$ for nonnegative w. Taking $E_{\lambda}$ on both sides of (4.20) followed by Jensen's inequality, since $\mu^{\delta}$ is a probability measure, and interchanging order of integration thanks to the integrable factor $e^{-\frac{\varepsilon^{2}}{2}|\xi|^{2}}$ turn (4.20) into

$$
\begin{gathered}
2(D(\varepsilon) \delta)^{2} P_{\lambda}\left\{A^{\delta}\right\}+2 e^{2 l N} \int_{\mathbb{R}_{i}^{N}} E_{\lambda}\left\{\left[N_{b}\left(\Theta^{\delta}, Z_{\Theta^{\delta}}\right)\right]^{2}\right\} \kappa^{i}(d b) \\
\geq(2 \pi)^{-2 d}\left(\int_{\mathbb{R}^{d}} e^{-\frac{\varepsilon^{2}}{2}|\xi|^{2}} Q_{\mu^{\delta}}^{Y}(\xi) Q_{\kappa^{i}}^{X}(\xi) d \xi\right)^{2} P_{\lambda}\left\{A^{\delta}\right\} .
\end{gathered}
$$

(Recall that $P_{\lambda}\left\{\widetilde{A}^{\delta}\right\}=P_{\lambda}\left\{A^{\delta}\right\}$.) Using (4.14) we have

$$
\begin{gathered}
2(D(\varepsilon) \delta)^{2} \lambda_{d}[B(0, R)]+2 e^{2 l N} \int_{\mathbb{R}_{i}^{N}} E_{\lambda}\left\{\left[N_{b}\left(\Theta^{\delta}, Z_{\Theta^{\delta}}\right)\right]^{2}\right\} \kappa^{i}(d b) \\
\geq(2 \pi)^{-2 d}\left(\int_{\mathbb{R}^{d}} e^{-\frac{\varepsilon^{2}}{2}|\xi|^{2}} Q_{\mu^{\delta}}^{Y}(\xi) Q_{\kappa^{i}}^{X}(\xi) d \xi\right)^{2} E\left\{\lambda_{d}\left(Z\left(G \times[0, l]_{i}^{N}\right) \cap B(0, R)\right)\right\} .
\end{gathered}
$$


Right now the issue that remains is how to change the term $\int_{\mathbb{R}_{i}^{N}} E_{\lambda}\left\{\left[N_{b}\left(\Theta^{\delta}\right.\right.\right.$, $\left.\left.\left.Z_{\Theta^{\delta}}\right)\right]^{2}\right\} \kappa^{i}(d b)$. Let $\left\{\theta_{1}, \theta_{2}, \cdots, \theta_{k}, \cdots\right\} \subset\left[G^{\delta} \cap \mathbb{Q}^{n}\right] \times\left[[0, l]_{i}^{N} \cap \mathbb{Q}^{N}\right]$ be the possible values of $\Theta^{\delta}$ with the probability $\pi_{k}$ under $P^{0}(=P)$ that $\Theta^{\delta}$ takes $\theta_{k}$. Since $\Theta^{\delta}$ is independent of $Z^{l_{1}}, \Theta^{\delta}$ is independent of $Z^{l_{1}}+x$ as well for all $x \in \mathbb{R}^{d}$ under $P^{0}$, where $Z^{l_{1}}+x$ is the field $\left\{Z_{t}+x, t \in J\left(l_{1}\right)\right\}$. Also note that $\Theta^{\delta}$ takes values in $J\left(l_{1}\right)$. It follows that

$$
\begin{gathered}
E_{\lambda}\left\{\left[N\left(\Theta^{\delta}, Z_{\Theta^{\delta}}\right)\right]^{2}\right\}=\int_{\mathbb{R}^{d}} E^{x}\left\{\left[N\left(\Theta^{\delta}, Z_{\Theta^{\delta}}\right)\right]^{2}\right\} d x \\
=\int_{\mathbb{R}^{d}} E\left\{\left[N\left(\Theta^{\delta}, Z_{\Theta^{\delta}}+x\right)\right]^{2}\right\} d x=\int_{\mathbb{R}^{d}} \sum_{k=1}^{\infty} \pi_{k} E\left\{\left[N\left(\theta_{k}, Z_{\theta_{k}}+x\right)\right]^{2}\right\} d x \\
=\sum_{k=1}^{\infty} \pi_{k} \int_{\mathbb{R}^{d}} E\left\{\left[N\left(\theta_{k}, Z_{\theta_{k}}+x\right)\right]^{2}\right\} d x=\sum_{k=1}^{\infty} \pi_{k} E_{\lambda}\left\{\left[N\left(\theta_{k}, Z_{\theta_{k}}\right)\right]^{2}\right\} .
\end{gathered}
$$

The following conditional expectation representation of $P_{s, t} f\left(Z_{s}\right)$ is of some interest.

For all $s, t \in \mathbb{R}^{n+N}$ and all continuous functions $f: \mathbb{R}^{d} \rightarrow \mathbb{R}_{+}$in $L^{1}$,

$$
P_{s, t} f\left(Z_{s}\right)=E_{\lambda}\left[f\left(Z_{t}\right) \mid Z_{s}\right] P_{\lambda}-\text { a.s., }
$$

where the $P_{\lambda}$-null set in (4.24) can be made independent of $t$.

Verification of (4.24). For all nonnegative functions $h$, by Fubini's theorem, a change of variable and the fact that Lebesgue measure is translation-invariant,

$$
E_{\lambda}\left[h\left(Z_{t}\right)\right]=\int_{\mathbb{R}^{d}} E\left[h\left(x+Z_{t}\right)\right] d x=\int_{\mathbb{R}^{d}} h(x) d x .
$$

In particular, for all $t \in \mathbb{R}^{n+N}$ and Borel sets $B$ in $\mathbb{R}^{d}, P_{\lambda}\left(Z_{t} \in B\right)=\lambda_{d}(B)$. That is, under $P_{\lambda}$, the distribution of $Z_{t}$ is $\lambda_{d}$ for all $t \in \mathbb{R}^{n+N}$. Let $g$ be a bounded measurable function. Then

$$
E_{\lambda}\left[f\left(Z_{t}\right) g\left(Z_{s}\right)\right]=\int_{\mathbb{R}^{d}} E\left[f\left(Z_{t}+x\right) g\left(Z_{s}+x\right)\right] d x=\int_{\mathbb{R}^{d}} g(y) E\left[f\left(Z_{t}-Z_{s}+y\right)\right] d y .
$$

Since the distribution of $Z_{s}$ is $\lambda_{d}$ under $P_{\lambda},(4.24)$ follows. The assertion that there is a null set independent of $t$ is quite clear beacuse $Z$ is rcll and $\mathbb{Q}^{n+N}$ is dense in $\mathbb{R}^{n+N}$.

(4.24) implies that

$$
E_{\lambda}\left\{\left[N_{b}\left(\theta_{k}, Z_{\theta_{k}}\right)\right]^{2}\right\}=E_{\lambda}\left\{\left(E_{\lambda}\left[\int_{\mathbb{R}^{n+N}} f^{\varepsilon}\left(Z_{t}\right) \nu_{(b)}^{\delta}(d t) \mid Z_{\theta_{k}}\right]\right)^{2}\right\} .
$$

It follows from conditional Jensen's inequality under $P_{\lambda}$ that

$$
E_{\lambda}\left\{\left[N_{b}\left(\theta_{k}, Z_{\theta_{k}}\right)\right]^{2}\right\} \leq E_{\lambda}\left\{\left[\int_{\mathbb{R}^{n+N}} f^{\varepsilon}\left(Z_{t}\right) \nu_{(b)}^{\delta}(d t)\right]^{2}\right\} .
$$

(4.23) and (4.25) imply that

$$
E_{\lambda}\left\{\left[N_{b}\left(\Theta^{\delta}, Z_{\Theta^{\delta}}\right)\right]^{2}\right\} \leq E_{\lambda}\left\{\left[\int_{\mathbb{R}^{n+N}} f^{\varepsilon}\left(Z_{t}\right) \nu_{(b)}^{\delta}(d t)\right]^{2}\right\} .
$$


Next, we show that

$$
E_{\lambda}\left\{\left[\int_{\mathbb{R}^{n+N}} f^{\varepsilon}\left(Z_{t}\right) \nu_{(b)}^{\delta}(d t)\right]^{2}\right\}=(2 \pi)^{-d} \int_{\mathbb{R}^{d}} e^{-\varepsilon^{2}|\xi|^{2}} Q_{\nu_{(b)}^{\delta}}^{Z}(\xi) d \xi .
$$

Proof of (4.27). As in the proof of (4.24), we find that for all $s, t \in \mathbb{R}^{n+N}$,

$$
E_{\lambda}\left\{f^{\varepsilon}\left(Z_{s}\right) f^{\varepsilon}\left(Z_{t}\right)\right\}=\int_{\mathbb{R}^{d}}\left(P_{s, t} f^{\varepsilon}(y)\right) f^{\varepsilon}(y) d y .
$$

By (4.3) and Fubini's theorem, thanks to $f^{\varepsilon}, \widehat{f^{\varepsilon}} \in L^{2}$,

$$
\begin{aligned}
& E_{\lambda}\left\{f^{\varepsilon}\left(Z_{s}\right) f^{\varepsilon}\left(Z_{t}\right)\right\}=\int_{\mathbb{R}^{d}}\left(P_{s, t} f^{\varepsilon}(y)\right) f^{\varepsilon}(y) d y \\
& =\int_{\mathbb{R}^{d}}\left((2 \pi)^{-d} \int_{\mathbb{R}^{d}} e^{i y \cdot \xi \widehat{f^{\varepsilon}}}(-\xi) E e^{i \xi \cdot\left(Z_{t}-Z_{s}\right)} d \xi\right) f^{\varepsilon}(y) d y \\
& =(2 \pi)^{-d} \int_{\mathbb{R}^{d}}\left|\widehat{f^{\varepsilon}}(\xi)\right|^{2} E e^{i \xi \cdot\left(Z_{t}-Z_{s}\right)} d \xi .
\end{aligned}
$$

Thus, by Fubini's theorem,

$$
\begin{aligned}
& E_{\lambda}\left\{\left[\int_{\mathbb{R}^{n+N}} f^{\varepsilon}\left(Z_{t}\right) \nu_{(b)}^{\delta}(d t)\right]^{2}\right\} \\
& =E_{\lambda}\left\{\int_{\mathbb{R}^{n+N}} \int_{\mathbb{R}^{n+N}} f^{\varepsilon}\left(Z_{s}\right) f^{\varepsilon}\left(Z_{t}\right) \nu_{(b)}^{\delta}(d s) \nu_{(b)}^{\delta}(d t)\right\} \\
& =\int_{\mathbb{R}^{n+N}} \int_{\mathbb{R}^{n+N}} E_{\lambda}\left\{f^{\varepsilon}\left(Z_{s}\right) f^{\varepsilon}\left(Z_{t}\right)\right\} \nu_{(b)}^{\delta}(d s) \nu_{(b)}^{\delta}(d t) \\
& =\int_{\mathbb{R}^{n+N}} \int_{\mathbb{R}^{n+N}}\left((2 \pi)^{-d} \int_{\mathbb{R}^{d}}|\widehat{f} \varepsilon(\xi)|^{2} E e^{i \xi \cdot\left(Z_{t}-Z_{s}\right)} d \xi\right) \nu_{(b)}^{\delta}(d s) \nu_{(b)}^{\delta}(d t) \\
& =(2 \pi)^{-d} \int_{\mathbb{R}^{d}}\left|\widehat{f^{\varepsilon}}(\xi)\right|^{2} Q_{\nu_{(b)}^{Z}}^{Z}(\xi) d \xi=(2 \pi)^{-d} \int_{\mathbb{R}^{d}} e^{-\varepsilon^{2}|\xi|^{2}} Q_{\nu_{(b)}^{\delta}}^{Z}(\xi) d \xi .
\end{aligned}
$$

(4.27) is proved.

Since $Z$ has stationary increments, for all $b \in \mathbb{R}^{N}$ and $\xi \in \mathbb{R}^{d}$,

$$
Q_{\nu_{(b)}^{\delta}}^{Z}(\xi)=Q_{\nu^{\delta}}^{Z}(\xi)
$$

(4.27) and (4.28) imply that

$$
E_{\lambda}\left\{\left[\int_{\mathbb{R}^{n+N}} f^{\varepsilon}\left(Z_{t}\right) \nu_{(b)}^{\delta}(d t)\right]^{2}\right\}=(2 \pi)^{-d} \int_{\mathbb{R}^{d}} e^{-\varepsilon^{2}|\xi|^{2}} Q_{\mu^{\delta}}^{Y}(\xi) Q_{\kappa^{i}}^{X}(\xi) d \xi .
$$

Note that $e^{-\varepsilon^{2}|\xi|^{2}} \leq e^{-\frac{\varepsilon^{2}}{2}|\xi|^{2}}$ while $Q_{\mu^{\delta}}^{Y}(\xi) Q_{\kappa^{i}}^{X}(\xi) \in[0,1]$. It follows from (4.26) and (4.29) that

$$
E_{\lambda}\left\{\left[N_{b}\left(\Theta^{\delta}, Z_{\Theta^{\delta}}\right)\right]^{2}\right\} \leq(2 \pi)^{-d} \int_{\mathbb{R}^{d}} e^{-\frac{\varepsilon^{2}}{2}|\xi|^{2}} Q_{\mu^{\delta}}^{Y}(\xi) Q_{\kappa^{i}}^{X}(\xi) d \xi
$$


Note that the right hand side of (4.30) is independent of $b$. We plug (4.30) into (4.22) and deduce that

$$
2(D(\varepsilon) \delta)^{2} \lambda_{d}[B(0, R)]+2 e^{2 l N}(2 \pi)^{-d} \int_{\mathbb{R}^{d}} e^{-\frac{\varepsilon^{2}}{2}|\xi|^{2}} Q_{\mu^{\delta}}^{Y}(\xi) Q_{\kappa^{i}}^{X}(\xi) d \xi
$$

$$
\geq(2 \pi)^{-2 d}\left(\int_{\mathbb{R}^{d}} e^{-\frac{\varepsilon^{2}}{2}|\xi|^{2}} Q_{\mu^{\delta}}^{Y}(\xi) Q_{\kappa^{i}}^{X}(\xi) d \xi\right)^{2} E\left\{\lambda_{d}\left(Z\left(G \times[0, l]_{i}^{N}\right) \cap B(0, R)\right)\right\} .
$$

Part IV. Choose any sequence $\delta_{k} \downarrow 0$ as $k \rightarrow \infty$ where $k=1,2, \cdots$. Since $G^{\delta_{1}}$ is bounded, there exists a probability measure $\mu$ on $G$ such that along some subsequence $\delta_{m} \rightarrow 0, \mu^{\delta_{m}} \rightarrow \mu$ weakly. To see that $\mu$ is on $G$, we notice that $G$, as well as each $G^{\delta}$, is compact and that $G \subset G^{\delta_{m+1}} \subset G^{\delta_{m}}$. Taking the indicator function $1_{G^{\delta}}$ and noting that $\mu^{\delta_{m}}$ is on $G^{\delta_{m}}$, we can easily find a contradiction if $\mu$ has a positive mass on a compact set $B$ with $B \cap G=\emptyset$. We can write

$$
\int_{\mathbb{R}^{d}} e^{-\frac{\varepsilon^{2}}{2}|\xi|^{2}} Q_{\mu^{\delta_{m}}}^{Y}(\xi) Q_{\kappa^{i}}^{X}(\xi) d \xi=\int_{\mathbb{R}^{n}} \int_{\mathbb{R}^{n}} f(s, t) \mu^{\delta_{m}}(d s) \mu^{\delta_{m}}(d t),
$$

where

$$
f(s, t)=\int_{\mathbb{R}^{d}} e^{-\frac{\varepsilon^{2}}{2}|\xi|^{2}} H(s, t, \xi) Q_{\kappa^{i}}^{X}(\xi) d \xi .
$$

Since $|H| \leq 1$ and $H$ is continuous, $f$ is a bounded continuous function. Since $\mu^{\delta_{m}} \rightarrow \mu$ weakly,

$$
\lim _{m \rightarrow \infty} \int_{\mathbb{R}^{n}} \int_{\mathbb{R}^{n}} f(s, t) \mu^{\delta_{m}}(d s) \mu^{\delta_{m}}(d t)=\int_{\mathbb{R}^{n}} \int_{\mathbb{R}^{n}} f(s, t) \mu(d s) \mu(d t) .
$$

In other words,

$$
\lim _{m \rightarrow \infty} \int_{\mathbb{R}^{d}} e^{-\frac{\varepsilon^{2}}{2}|\xi|^{2}} Q_{\mu^{\delta_{m}}}^{Y}(\xi) Q_{\kappa^{i}}^{X}(\xi) d \xi=\int_{\mathbb{R}^{d}} e^{-\frac{\varepsilon^{2}}{2}|\xi|^{2}} Q_{\mu}^{Y}(\xi) Q_{\kappa^{i}}^{X}(\xi) d \xi>0 .
$$

The integral to the right in (4.32) must be strictly positive for any probability measure $\nu \times \kappa^{i}$ because otherwise

$$
\int_{\mathbb{R}^{d}} Q_{\nu}^{Y}(\xi) Q_{\kappa^{i}}^{X}(\xi) d \xi=0
$$

would imply that $\widehat{O}_{\nu \times \kappa^{i}}=0$, where

$$
O_{\nu \times \kappa^{i}}(E)=\int_{\mathbb{R}^{n+N}} 1\left(Z_{t} \in E\right)\left(\nu \times \kappa^{i}\right)(d t), E \in \mathcal{B}\left(\mathbb{R}^{d}\right) .
$$

Also note that

$$
\int_{\mathbb{R}^{d}} e^{-\frac{\varepsilon^{2}}{2}|\xi|^{2}} Q_{\mu^{\delta}}^{Y}(\xi) Q_{\kappa^{i}}^{X}(\xi) d \xi \leq \int_{\mathbb{R}^{d}} e^{-\frac{\varepsilon^{2}}{2}|\xi|^{2}} d \xi<\infty .
$$

Replacing $\delta$ by $\delta_{m}$ in (4.31) and letting $m \rightarrow \infty$ show that

$$
\int_{\mathbb{R}^{d}} e^{-\frac{\varepsilon^{2}}{2}|\xi|^{2}} Q_{\mu}^{Y}(\xi) Q_{\kappa^{i}}^{X}(\xi) d \xi \leq \frac{2 e^{2 l N}(2 \pi)^{d}}{E\left\{\lambda_{d}\left(Z\left(G \times[0, l]_{i}^{N}\right) \cap B(0, R)\right)\right\}} .
$$

Finally, by Fatou's lemma, letting $\varepsilon \rightarrow 0$ in (4.33) concludes the proof of (4.6). 
Let us take a good look at our proof one more time and ask the following question. Have we really used the assumption that $Y$ has stationary increments? We did say " $Z$ has stationary increments" once, but in fact we can get what we want as long as $X$ has stationary increments regardless of $Y$. To make this modification, we have only two more things to prove.

A. Let $P_{s, t} f(x)=E\left[f\left(Z_{t}-Z_{s}+x\right)\right], x \in \mathbb{R}^{d}, t, s \in \mathbb{R}^{n+N}, f: \mathbb{R}^{d} \rightarrow \mathbb{R}_{+}$, where $Z_{(p, q)}=Y_{p}+X_{q}, p \in \mathbb{R}^{n}, q \in \mathbb{R}^{N}, Y, X$ independent. Let $a=(v, b), v \in \mathbb{R}^{n}, b \in$ $\mathbb{R}_{i}^{N}, \alpha=(v, \bar{b}), \bar{b} \in \mathbb{R}_{i}^{N}$. Assume that $X$ has stationary increments. We claim that the following partial stationary increment property holds:

$$
P_{a, t} f=P_{\alpha, t-a+\alpha} f, \forall t=(p, q) .
$$

We have $t-a+\alpha=(p, q-b+\bar{b})$, and $\left(Y_{p}+X_{q-b+\bar{b}}\right)-\left(Y_{v}+X_{\bar{b}}\right) \stackrel{d}{=} Y_{p}-Y_{v}+X_{q-b}$. On the other hand, $\left(Y_{p}+X_{q}\right)-\left(Y_{v}+X_{b}\right) \stackrel{d}{=} Y_{p}-Y_{v}+X_{q-b}$. Our claim follows.

B. Let $\nu^{\delta}=\mu^{\delta} \times \kappa^{i}$, where $\mu^{\delta}$ is some probability measure on $\mathbb{R}^{n}$ and $\kappa^{i}$ on $\mathbb{R}^{N}$. Let $\nu_{(b)}^{\delta}=\nu_{(0, b)}^{\delta}, b \in \mathbb{R}_{i}^{N}$. Then $\nu_{(b)}^{\delta}=\mu^{\delta} \times \kappa_{(b)}^{i}$. Since $X$ has stationary increments and is independent of $Y$, we get $Q_{\nu_{(b)}^{\delta}}^{Z}(\xi)=Q_{\nu^{\delta}}^{Z}(\xi)$.

Thus, (4.4) still holds after the stationary increments assumption on $Y$ is removed. The proof of part (iii) is complete first. We are now in business. Let $Y$ be the deterministic field $\mathcal{I}_{t}=t, t \in \mathbb{R}^{d}, d=n$, and $G=F \subset \mathbb{R}^{d}$. We have $F=\mathcal{I}(F)$, $E e^{i \xi \cdot\left(\mathcal{I}_{x}-\mathcal{I}_{y}\right)}=e^{i \xi \cdot(x-y)}$, which is clearly a continuous function of $(x, y, \xi)$, and $Q_{\mu}^{\mathcal{I}}(\xi)=|\hat{\mu}(\xi)|^{2}, \xi \in \mathbb{R}^{d}$, for all probability measures $\mu$ in $\mathbb{R}^{d}$. Plainly, $\mathcal{I}$ is an rcll field continuous in probability and that happens to have stationary increments. It is totally trivial to construct $\mathbf{b}$ for a deterministic rcll field. Let $\theta$ be a deterministic rcll mapping from $\mathbb{R}^{N}$ into $\mathbb{R}^{d}$, namely a deterministic field. Let $\mathbf{b}(\omega)=\omega, \omega \in \Omega$. Let $\Omega^{\prime}=\Omega$. Obviously, for any kind of domain for $\theta^{l}, \mathbf{b}$ is independent of $\theta^{l}$ and $r\left(\Omega^{\prime}\right)=\Omega_{l}$, and $r(\mathbf{b}(\omega))=r(\omega)$ for each $\omega \in \Omega^{\prime}$. [Remark: Part (ii) of Proposition 4.1 implies that if $\theta$ is continuous, then given any Borel set $G \subset \mathbb{R}^{N}$,

$$
\lambda_{d}(\theta(G))>0 \Longrightarrow \int_{\mathbb{R}^{d}} Q_{\eta}^{\theta}(\xi) d \xi<\infty \text { for some } \eta \in \mathcal{P}(G),
$$

where $Q_{\eta}^{\theta}(\xi)=\int_{\mathbb{R}^{N}} \int_{\mathbb{R}^{N}} e^{i \xi \cdot\left(\theta_{s}-\theta_{t}\right)} \eta(d s) \eta(d t)$. In fact conditions on $\theta$ can be weakened. The above result is still valid provided $e^{i \xi \cdot\left(\theta_{s}-\theta_{t}\right)}$ can be approximated by simple functions for rcll $\theta$ for example.]

(i) follows from letting $Y=\mathcal{I}$ in (4.4) and (ii) follows from setting $X$ to the deterministic field 0 in (4.4).

Remark on Proposition 4.1. In the case that $X$ has $\mathbf{b}$ on $\mathbb{R}^{N}$, part (i) and part (iii) of the proposition are still valid if we replace $\mathbb{R}_{i}^{N}$ by $\mathbb{R}^{N}$ and $\kappa^{i}$ by $\tilde{\kappa}$ simultaneously. Recall that $\tilde{\kappa}(d t)=2^{-N} e^{-\sum_{j=1}^{N}\left|t_{j}\right|} d t, t=\left(t_{1}, \cdots, t_{N}\right) \in \mathbb{R}^{N}$. To see this, note that if $\bar{b} \in[-l, l]^{N}$, then $\tilde{\kappa}_{(b-\bar{b})}(\bullet) \leq e^{l N} \tilde{\kappa}_{(b)}(\bullet)$ for all $b \in \mathbb{R}^{N}$. This inequality is easy to understand. The probability measure $\tilde{\kappa}$ is also related to potential theory for fields with stationary increments. Let $f_{i j}(\xi)=\int_{\mathbb{R}_{i}^{N}} \int_{\mathbb{R}_{j}^{N}} E e^{i \xi \cdot\left(X_{s}-X_{t}\right)} \tilde{\kappa}(d s) \tilde{\kappa}(d t)$. Then $Q_{\tilde{\kappa}}^{X}=\sum_{i, j} f_{i j}$ with $\left|f_{i j}\right| \leq 2^{-2 N}$. Note that $f_{i i} \in\left[0,2^{-2 N}\right]$. The kernel $Q_{\tilde{\kappa}}^{X}$ is not good for use in general, since these nonsymmetric $\mathbb{C}$-valued cross terms $f_{i j}$, where $i \neq j$, are hard to evaluate. This is true even if $X$ is symmetric. If $X$ is symmetric, then $f_{i j} \geq 0$ for all $i, j$. Thus, $Q_{\eta}^{Y} Q_{\tilde{\kappa}}^{X} \in L^{1}$ iff every $Q_{\eta}^{Y} f_{i j} \in L^{1}$. In this case, the proposition can be proved in another way. We only have to prove it for $\tilde{\kappa}$. If 
$E\left\{\lambda_{d}\left(X\left(\mathbb{R}_{i}^{N}\right) \pm Y(G)\right)\right\}>0$, then $E\left\{\lambda_{d}\left(X\left(\mathbb{R}^{N}\right) \pm Y(G)\right)\right\}>0$, which implies that $Q_{\eta}^{Y} Q_{\tilde{\kappa}}^{X} \in L^{1}$. Thus, $Q_{\eta}^{Y} f_{i i} \in L^{1}$ for each $i$. If $X$ is not symmetric this short cut fails, because $Q_{\tilde{\kappa}}^{X} \geq f_{i i}$ does not necessarily hold.

Proposition 4.2. Let $X: \mathbb{R}^{N} \rightarrow \mathbb{R}^{d}$ be a field. Let $G \subset \mathbb{R}^{N}$ be a Borel set. If $\int_{\mathbb{R}^{d}} Q_{\eta}^{X}(\xi) d \xi<\infty$ for some $\eta \in \mathcal{P}(G)$, then

$$
E\left\{\lambda_{d}(X(G))\right\} \geq(2 \pi)^{d}\left(\int_{\mathbb{R}^{d}} Q_{\eta}^{X}(\xi) d \xi\right)^{-1} .
$$

Proof. By Fubini's theorem,

$$
Q_{\eta}^{X}(\xi)=E\left|\int_{\mathbb{R}^{N}} e^{i \xi \cdot X_{t}} \eta(d t)\right|^{2}=E\left\{\int_{\mathbb{R}^{N}} \int_{\mathbb{R}^{N}} e^{i \xi \cdot\left(X_{s}-X_{t}\right)} \eta(d s) \eta(d t)\right\},
$$

which also shows that $Q_{\eta}^{X}(\xi) \in[0,1]$ since $\left|\int_{\mathbb{R}^{N}} e^{i \xi \cdot X_{t}} \eta(d t)\right| \leq 1$. Introduce the $\eta$-occupation measure $O_{\eta}$ by

$$
\int_{\mathbb{R}^{d}} f(x) O_{\eta}(d x)=\int_{\mathbb{R}^{N}} f\left(X_{t}\right) \eta(d t),
$$

where $f: \mathbb{R}^{d} \rightarrow \mathbb{R}_{+}$is a measurable function. In particular,

$$
O_{\eta}(E)=\int_{\mathbb{R}^{N}} 1\left(X_{t} \in E\right) \eta(d t), E \in \mathcal{B}\left(\mathbb{R}^{d}\right) .
$$

Note that $O_{\eta} \in \mathcal{P}(X(G))$ since $\eta \in \mathcal{P}(G)$. From the very definition of $O_{\eta}$, we have a frequently used the identity

$$
\int_{\mathbb{R}^{d}} e^{i \xi \cdot x} O_{\eta}(d x)=\int_{\mathbb{R}^{N}} e^{i \xi \cdot X_{t}} \eta(d t) .
$$

By (4.36),

$$
\left|\widehat{O}_{\eta}(\xi)\right|^{2}=\int_{\mathbb{R}^{N}} \int_{\mathbb{R}^{N}} e^{i \xi \cdot\left(X_{t}-X_{s}\right)} \eta(d t) \eta(d s) .
$$

It follows from (4.37) and (4.35) that

$$
Q_{\eta}^{X}(\xi)=E\left|\widehat{O}_{\eta}(\xi)\right|^{2} .
$$

In the following, $\|f\|_{L^{2}\left(\mathbb{R}^{d}\right)}^{2}=\int_{\mathbb{R}^{d}}|f(\xi)|^{2} d \xi$ for any complex-valued function $f$. Since

$$
\left\|\widehat{O}_{\eta}\right\|_{L^{2}\left(\mathbb{R}^{d}\right)}^{2}=\int_{\mathbb{R}^{d}}\left|\widehat{O}_{\eta}(\xi)\right|^{2} d \xi
$$

the Fubini theorem and (4.38) imply that

$$
E\left\|\widehat{O}_{\eta}\right\|_{L^{2}\left(\mathbb{R}^{d}\right)}^{2}=\int_{\mathbb{R}^{d}} Q_{\eta}^{X}(\xi) d \xi<\infty .
$$

By (4.39), $\left\|\widehat{O}_{\eta}\right\|_{L^{2}\left(\mathbb{R}^{d}\right)}^{2}<\infty$ a.s. By Plancherel's theorem, the measure $O_{\eta}$ is absolutely continuous with respect to $\lambda_{d}$ a.s. and there exists a measurable version $L_{\eta}(x)$ of the density (also called the $\eta$-local time) of $O_{\eta}$ satisfying

$$
\left\|L_{\eta}\right\|_{L^{2}\left(\mathbb{R}^{d}\right)}^{2}=(2 \pi)^{-d}\left\|\widehat{O}_{\eta}\right\|_{L^{2}\left(\mathbb{R}^{d}\right)}^{2} \text { a.s. }
$$


Note that $O_{\eta}(A)=\int_{A} L_{\eta}(x) d x, A \in \mathcal{B}\left(\mathbb{R}^{d}\right)$. Since $O_{\eta} \in \mathcal{P}(X(G)), \operatorname{supp}\left(L_{\eta}\right) \subset$ $\overline{X(G)}$. Also note that $O_{\eta}\left(\mathbb{R}^{d}\right)=1$. Thus, by the Cauchy-Schwarz inequality and $(4.40)$

$$
\begin{aligned}
& 1=\left(O_{\eta}\left(\mathbb{R}^{d}\right)\right)^{2}=\left(\int_{\mathbb{R}^{d}} 1_{X(G)}(x) L_{\eta}(x) d x\right)^{2} \\
& \leq \int_{\mathbb{R}^{d}} 1_{X(G)}^{2}(x) d x \int_{\mathbb{R}^{d}} L_{\eta}^{2}(x) d x \\
& =\lambda_{d}(X(G))(2 \pi)^{-d}\left\|\widehat{O}_{\eta}\right\|_{L^{2}\left(\mathbb{R}^{d}\right)}^{2} \quad \text { a.s. }
\end{aligned}
$$

Applying the inequality $E\left(\zeta^{-1}\right) E \zeta \geq 1$ valid for all positive random variables $\zeta$ for the above display followed by (4.39) yields

$$
E\left\{\lambda_{d}(X(G))\right\} \geq(2 \pi)^{d}\left[\int_{\mathbb{R}^{d}} Q_{\eta}^{X}(\xi) d \xi\right]^{-1}>0 .
$$

The remaining portion of this last section is devoted to constructing $\mathbf{b}$ for random fields selected for their importance in the current research and for their roles as the fundamental structural blocks to which many other fields are adapted.

Additive fields. A field $X: \mathbb{R}^{N} \rightarrow \mathbb{R}^{d}$ with $X_{0}=0$ a.s. is called the additive field if $X$ has independent increments in the usual partial order $\preceq$. An additive field is called the Lévy field if it also has stationary increments. It is easy to see that Lévy fields are Markov fields. Clearly additive Lévy processes are Lévy fields. (See below for the precise definition of the additive Lévy process.) But there are many Lévy fields which are not additive Lévy processes. For each $\alpha \in(0,1)$, we define a field $\sigma^{\alpha}: \mathbb{R}_{+}^{N} \rightarrow \mathbb{R}_{+}^{N}$ as

$\sigma_{u}^{\alpha}=\sigma_{u_{1}}^{1}+\sigma_{u_{2}}^{2}+\cdots+\sigma_{u_{N}}^{N}, u \in \mathbb{R}_{+}^{N}, \sigma_{u_{j}}^{j}=\left(\sigma_{u_{j}}^{j, 1}, \sigma_{u_{j}}^{j, 2}, \cdots, \sigma_{u_{j}}^{j, N}\right), j=1,2, \cdots, N$, where the $\sigma_{u_{j}}^{j, l}, 1 \leq j \leq N, 1 \leq l \leq N$, are $N^{2}$ i.i.d. standard $\alpha$-stable subordinators with one common Laplace exponent $\lambda^{\alpha}$. We extend $\sigma^{\alpha}$ to $\tilde{\sigma}^{\alpha}: \mathbb{R}^{N} \rightarrow \mathbb{R}^{N}$ as follows. Let $\zeta^{j}$ be an independent copy of $\sigma^{j}$, where $\zeta^{j}$ is in the lcrl version, and define $\tilde{\sigma}_{t}^{j}=\sigma_{t}^{j}$ if $t \geq 0$ and $\tilde{\sigma}_{t}^{j}=-\zeta_{-t}^{j}$ if $t<0$. The $\zeta^{j}$ are chosen to be independent of one another and totally independent of $\sigma^{\alpha}$. Define

$$
\tilde{\sigma}_{t}^{\alpha}=\tilde{\sigma}_{t_{1}}^{1}+\tilde{\sigma}_{t_{2}}^{2}+\cdots+\tilde{\sigma}_{t_{N}}^{N}: \mathbb{R}^{N} \rightarrow \mathbb{R}^{N}, t=\left(t_{1}, t_{2}, \cdots, t_{N}\right) \in \mathbb{R}^{N} .
$$

$\tilde{\sigma}^{\alpha}$ is then an rcll field. It is easy to check that $\tilde{\sigma}_{t_{1}}^{\alpha} \preceq \tilde{\sigma}_{t_{2}}^{\alpha} \preceq \cdots \preceq \tilde{\sigma}_{t_{k}}^{\alpha}$ for any increasing sequence $t_{1} \preceq t_{2} \preceq \cdots \preceq t_{k}, t_{i} \in \mathbb{R}^{N}$. Therefore, if $X$ is rcll, then so too is the composition $X \circ \tilde{\sigma}^{\alpha}$, where $\tilde{\sigma}^{\alpha}$ is independent of $X$. Let $X: \mathbb{R}^{N} \rightarrow \mathbb{R}^{d}$ be a Lévy field. One can show that $X \circ \tilde{\sigma}^{\alpha}$ is also a Lévy field. For instance, in general $X \circ \tilde{\sigma}^{\alpha}$ is no longer an additive Lévy process, where $X$ is an additive Lévy process per se.

Assume that $X$ is an additive field in the rcll version and in the canonical form, i.e., $X_{t}(\omega)=\omega(t)$. We show that $X$ has $\mathbf{b}$ on $\mathbb{R}_{+}^{N}$.

Proof. Fix $l \in(0, \infty)$. Let $A=\{\omega \in \Omega: \omega(0)=0\}$. Then $P(A)=1$. Define a measurable mapping $\mathbf{b}_{A}: A \rightarrow \Omega_{+}$by $\mathbf{b}_{A}(\omega)(t)=\omega(t+L)-\omega(L), t \in \mathbb{R}_{+}^{N}$, for $\omega \in A$, where $L=(l, \cdots, l)$. Since $X$ is additive, $\mathbf{b}_{A}$ is independent of $X^{l}$, where $X^{l}$ is the field $\left\{X_{t}, t \in[0, l)^{N}\right\}$. Let $r$ be the restriction of $\Omega$ to $\Omega_{l}$, the space of rcll functions from $[0, l)^{N}$ into $\mathbb{R}^{d}$. Fix any $\omega \in r(A)$. We will extend $\omega$ to some 
$\omega^{\prime} \in A$ in the following way. Let $\omega^{\prime}(t)=\omega(t), t \in[0, L)$, and $\omega^{\prime}(t+L)=\omega(t), t \in$ $[0, L) \backslash\{0\}$, which implies that $\omega^{\prime}(L)=\omega(0)=0$ by the right-continuity of $\omega$. For $t \in \mathbb{R}^{N} \backslash([0, L) \cup[L, 2 L)), \omega^{\prime}(t)$ can be definded in any way possible as long as $\omega^{\prime}$ is rcll on $\mathbb{R}^{N}$ (since $\omega^{\prime}(0)=0, \omega^{\prime} \in A$ always) and ultimately some event $A^{\prime} \subset A$ is achieved after all $\omega \in r(A)$ are used. Obviously, $r\left(A^{\prime}\right)=r(A)$. For each $\omega^{\prime} \in A^{\prime}$, we have

$$
\mathbf{b}_{A}\left(\omega^{\prime}\right)(t)=\omega^{\prime}(t+L)-\omega^{\prime}(L)=\omega(t)-0=\omega(t)=\omega^{\prime}(t), t \in[0, L) .
$$

That is, $r\left[\mathbf{b}_{A}\left(\omega^{\prime}\right)\right]=r\left(\omega^{\prime}\right)$ for each $\omega^{\prime} \in A^{\prime}$. The proof that $X$ has $\mathbf{b}$ is complete.

In fact one can show that additive Lévy processes have $\mathbf{b}$ on $\mathbb{R}^{N}$. A process $Y_{t}, t \in \mathbb{R}_{+}$, with independent increments, rcll paths and values in $\mathbb{R}^{d}$ is called additive if $Y_{t}$ is continuous in probability and $Y_{0}=0$. An iterated additive process $X_{t}$ in $\mathbb{R}^{d}$ is a random field defined by

$$
X_{t}=X_{t_{1}}^{1}+X_{t_{2}}^{2}+\cdots+X_{t_{N}}^{N}, t=\left(t_{1}, t_{2}, \cdots, t_{N}\right) \in \mathbb{R}_{+}^{N},
$$

where the $X_{t_{j}}^{j}$ are independent additive processes in $\mathbb{R}^{d}$. When all the $X_{t_{j}}^{j}$ above are Lévy processes, $X_{t}$ is called an additive Lévy process. We extend $X$ to $\tilde{X}$ : $\mathbb{R}^{N} \rightarrow \mathbb{R}^{d}$ as follows. Let $\zeta^{j}$ be an independent copy of $X^{j}$ and define $\widetilde{X}_{t}^{j}=X_{t}^{j}$ if $t \geq 0$ and $\tilde{X}_{t}^{j}=-\zeta_{-t}^{j}$ if $t<0$. The $\zeta^{j}$ are chosen to be independent of one another and totally independent of $X$. Define

$$
\widetilde{X}_{t}=\tilde{X}_{t_{1}}^{1}+\tilde{X}_{t_{2}}^{2}+\cdots+\widetilde{X}_{t_{N}}^{N}: \mathbb{R}^{N} \rightarrow \mathbb{R}^{d}, t=\left(t_{1}, t_{2}, \cdots, t_{N}\right) \in \mathbb{R}^{N} .
$$

Here we will call $\tilde{X}$ iterated additive as well. Let $X: \mathbb{R}^{N} \rightarrow \mathbb{R}^{d}$ be an iterated additive process. Then $X$ has an rcll version in the usual partial order with $X_{0}=0$ a.s. since the $X^{j}$ are continuous in probability and each $\zeta^{j}$ can be taken in its lcrl version. Clearly, $X$ is an additive field. Assume that $X$ is in the canonical form. We just showed that $X$ has $\mathbf{b}$ on $\mathbb{R}_{+}^{N}$. We will show that $X$ also has $\mathbf{b}$ on $\mathbb{R}^{N}$.

Proof. By Lemma 2.1 (cf. $Z^{4}$ ), we only have to validate our claim in the oneparameter case: $X_{t}=Y_{t}^{1}$ if $t \geq 0$ and $X_{t}=-Y_{-t}^{2}$ if $t<0$, where $Y^{1}$ and $Y^{2}$ are i.i.d. additive processes, $Y^{1}$ is in the rcll version while $Y^{2}$ is in the lcrl version. We will therefore assume that $X$ is rcll and canonical. Fix $l \in(0, \infty)$. Let $A=\{\omega \in \Omega: \omega(0)=0\}$. Then $P(A)=1$. Let $\Omega_{+}$be the space of rcll functions from $\mathbb{R}_{+}$into $\mathbb{R}^{d}$. Define a measurable mapping $\mathbf{b}_{A}^{+}: A \rightarrow \Omega_{+}$by $\mathbf{b}_{A}^{+}(\omega)(t)=$ $\omega(t+l)-\omega(l), t \in \mathbb{R}_{+}$for $\omega \in A$. Since $X$ is an additive process and since $l>0, \mathbf{b}_{A}^{+}$is independent of $X^{l}$, where $X^{l}$ is the field $\left\{X_{t}, t \in[-l, l)\right\}$. (In fact, $\mathbf{b}_{A}^{+}$is independent of the field $\left\{X_{t}, t \in[-\infty, l)\right\}$.) Let $\Omega_{-}$be the space of rcll functions from $(-\infty, 0)$ into $\mathbb{R}^{d}$. Define a measurable mapping $\mathbf{b}_{A}^{-}: A \rightarrow \Omega_{-}$ by $\mathbf{b}_{A}^{-}(\omega)(t)=\omega(t-l)-\omega\left((-l)_{-}\right), \quad t \in(-\infty, 0)$ for $\omega \in A$. Likewise, $\mathbf{b}_{A}^{-}$is independent of $X^{l}$, since $\mathbf{b}_{A}^{-}$is independent of the field $\left\{X_{t}, t \in[-l, \infty)\right\}$. We can now define a measurable mapping $\mathbf{b}_{A}: A \rightarrow \Omega$ by $\mathbf{b}_{A}(\omega)(t)=\mathbf{b}_{A}^{+}(\omega)(t)$ for $t \geq 0$ and $\mathbf{b}_{A}(\omega)(t)=\mathbf{b}_{A}^{-}(\omega)(t)$ for $t<0$, for each $\omega \in \Omega$. Since $\mathbf{b}_{A}^{+}$and $\mathbf{b}_{A}^{-}$are independent, $\mathbf{b}_{A}$ is independent of $X^{l}$.

Fix any $\omega \in r(A)$. We will extend $\omega$ to some $\omega^{\prime} \in A$ as follows. Let $\omega^{\prime}(t)=$ $\omega(t), t \in[0, l)$, and $\omega^{\prime}(t+l)=\omega(t), t \in(0, l)$, which implies that $\omega^{\prime}(l)=\omega(0)=0$ by the right-continuity of $\omega$. Let $\omega^{\prime}(t)=\omega(t), t \in[-l, 0)$, and $\omega^{\prime}(t-l)=\omega(t), t \in$ $[-l, 0)$, which implies that $\omega^{\prime}\left((-l)_{-}\right)=\omega\left(0_{-}\right)=0$ thanks to the fact that the left limits exist and to the regularity condition that $\omega$ is continuous at 0 with $\omega(0)=0$. 
For $s \in \mathbb{R} \backslash\{t: t \in[-2 l, 2 l)\}, \omega^{\prime}(s)$ can be defined in any possible way as long as $\omega^{\prime}$ is rcll on $\mathbb{R}$ (since $\omega^{\prime}(0)=0, \omega^{\prime} \in A$ always) and ultimately some event $A^{\prime} \subset A$ is achieved after all $\omega \in r(A)$ are used. Obviously, $r\left(A^{\prime}\right)=r(A)$. For each $\omega^{\prime} \in A^{\prime}$, we have

$$
\mathbf{b}_{A}\left(\omega^{\prime}\right)(t)=\mathbf{b}_{A}^{+}\left(\omega^{\prime}\right)(t)=\omega^{\prime}(t+l)-\omega^{\prime}(l)=\omega(t)-0=\omega(t)=\omega^{\prime}(t), t \in[0, l),
$$

$\mathbf{b}_{A}\left(\omega^{\prime}\right)(t)=\mathbf{b}_{A}^{-}\left(\omega^{\prime}\right)(t)=\omega^{\prime}(t-l)-\omega^{\prime}\left((-l)_{-}\right)=\omega(t)-0=\omega(t)=\omega^{\prime}(t), t \in[-l, 0)$.

That is, $r\left[\mathbf{b}_{A}\left(\omega^{\prime}\right)\right]=r\left(\omega^{\prime}\right)$ for each $\omega^{\prime} \in A^{\prime}$. It follows that $X$ has $\mathbf{b}$ with domain $[-l, l)$.

Let $\mathbf{x}: \mathbb{R}^{N} \rightarrow \mathbb{R}^{d}$ be a symmetric additive Lévy process with Lévy exponent $\left(\Psi_{1}, \cdots, \Psi_{N}\right)$, i.e., $\Psi_{j} \geq 0$. Indeed $\mathbf{x} \circ \tilde{\sigma}^{\alpha}$, where $\tilde{\sigma}^{\alpha}$ is independent of $\mathbf{x}$, is a Lévy field and hence has $\mathbf{b}$ on $\mathbb{R}_{+}^{N}$. But that falls short of deriving potential-theoretic results for $G \subset \mathbb{R}^{N}$. Actually, $\mathbf{x} \circ \tilde{\sigma}^{\alpha}$ is b-related on $\mathbb{R}^{N}$ (which is one of the basic steps for instance for computing the Hausdorff dimension of the inverse image of a symmetric Lévy sheet.) We offer a proof of this claim. Right now we are not ready to prove this.

Let $X: \mathbb{R}^{N} \rightarrow \mathbb{R}^{d}$ be an rcll field. We call $X$ an $M$-field if for each $N$-dimensional compact interval $I \subset(0, \infty)^{N}$, there is a countable dense subset $D$ of $I$ such that for each finite subset $\left\{q_{1}, \cdots, q_{p}\right\}$ of $D,\left(X_{q_{1}}, \cdots, X_{q_{p}}\right)$ has a joint density. Given an $N$-dimensional compact interval $I=\left[a_{1}, b_{1}\right] \times \cdots \times\left[a_{N}, b_{N}\right] \subset(0, \infty)^{N}$, we define $I^{\prime}=\left[a_{1}, b_{1}\right) \times \cdots \times\left[a_{N}, b_{N}\right)$. We can also define $M$-fields with respect to $I \subset\left(\mathbb{R}_{i}^{N}\right)^{o}, i \geq 2$. For instance, $\left(\mathbb{R}_{2}^{N}\right)^{o}=(-\infty, 0) \times(0, \infty)^{N-1}$.

Lemma 4.3. Let $X: \mathbb{R}^{N} \rightarrow \mathbb{R}^{d}$ be an rcll field. For each integer $p=1,2, \cdots$, define

$$
D_{p}=\left\{\left(s_{1}, \cdots, s_{p}\right): s_{i} \in \mathbb{R}^{N},\left(X_{s_{1}}, \cdots, X_{s_{p}}\right) \text { does not have a joint density }\right\} .
$$

If $\lambda_{N p}\left(D_{p}\right)=0$ for all $p$, then for any distinct points $t_{1}, \cdots, t_{p}$ of $(0, \infty)^{N},(X \circ$ $\left.\sigma_{t_{1}}^{\alpha}, \cdots, X \circ \sigma_{t_{p}}^{\alpha}\right)$ has a joint density. In particular, $X \circ \sigma^{\alpha}$ is an $M$-field.

Proof. Since $\sigma^{\alpha}$ is an additive Lévy process and since each $\sigma_{u_{j}}^{j, l}$ is an $\alpha$-stable subordinator, $\left(\sigma_{t_{1}}^{\alpha}, \cdots, \sigma_{t_{p}}^{\alpha}\right)$ has a joint density $r_{t_{1}, \cdots, t_{p}}\left(s_{1}, \cdots, s_{p}\right)$ for any distinct points $t_{1}, \cdots, t_{p}$ of $(0, \infty)^{N}$. Because $\lambda_{N p}\left(D_{p}\right)=0,\left(X \circ \sigma_{t_{1}}^{\alpha}, \cdots, X \circ \sigma_{t_{p}}^{\alpha}\right)$ has a joint density

$$
h_{t_{1}, \cdots, t_{p}}\left(x_{1}, \cdots, x_{p}\right)=\int \phi_{s_{1}, \cdots, s_{p}}\left(x_{1}, \cdots, x_{p}\right) r_{t_{1}, \cdots, t_{p}}\left(s_{1}, \cdots, s_{p}\right) d s_{1} \cdots d s_{p},
$$

where $\phi_{s_{1}, \cdots, s_{p}}\left(x_{1}, \cdots, x_{p}\right)$ is a joint density of $\left(X_{s_{1}}, \cdots, X_{s_{p}}\right)$ and $\left(s_{1}, \cdots, s_{p}\right) \notin$ $D_{p}$.

Lemma 4.4. Let $X: \mathbb{R}^{N} \rightarrow \mathbb{R}^{d}$ be an rcll field. Let $X^{n}: \mathbb{R}^{N} \rightarrow \mathbb{R}^{d}, n=$ $1,2, \cdots$, be a sequence of rcll fields on the same sample space $\Omega$, the space of rcll functions from $\mathbb{R}^{N}$ into $\mathbb{R}^{d}$, such that $X^{n} \stackrel{\text { law }}{\longrightarrow} X$. Fix an $N$-dimensional compact interval $I=\left[a_{1}, b_{1}\right] \times \cdots \times\left[a_{N}, b_{N}\right] \subset\left(\mathbb{R}_{2}^{N}\right)^{o}$. Assume that $X$ is an $M$-field on $I_{\varepsilon}=\left[a_{1}-\varepsilon, b_{1}+\varepsilon\right] \times\left[a_{2}-\varepsilon, b_{2}+\varepsilon\right] \times \cdots \times\left[a_{N}-\varepsilon, b_{N}+\varepsilon\right] \subset\left(\mathbb{R}_{2}^{N}\right)^{o}$ for some sufficiently small $\varepsilon>0$. Then for any compact $F \subset \mathbb{R}^{d}$,

$$
\liminf E\left\{\lambda_{d}\left[X^{n}(I)-F\right]\right\} \geq E\left\{\lambda_{d}[X(I)-F]\right\} .
$$


In particular,

$$
E\left\{\lambda_{d}[X(I)-F]\right\}>0 \Longrightarrow E\left\{\lambda_{d}\left[X^{n}(I)-F\right]\right\}>0 \text { for all large } n .
$$

(4.41) and (4.42) are still valid when $I$ is replaced by $I^{\prime}$.

Proof. Without loss of generality we can assume that $\Omega=\Omega_{\varepsilon}$, the space of rcll functions from $I_{\varepsilon}$ into $\mathbb{R}^{d}$. Let $P$ be the probability measure on $\Omega$ given by $X$, and $P_{n}$ be the probability measure on $\Omega$ given by $X^{n}$. So $P_{n} \rightarrow P$ weakly. Consider an event $B=\{\omega \in \Omega: \omega(I) \cap K \neq \emptyset\}$, where $K \subset \mathbb{R}^{d}$ is a compact set. Note that $B^{c}=\{\omega \in \Omega: \omega(I) \cap K=\emptyset\}$. Since $X$ is an $M$-field on $I_{\varepsilon}$ and since $K$ is compact, the standard approximation argument shows that $P(\bar{B})=P(B)$ and $P\left(\overline{B^{c}}\right)=P\left(B^{c}\right)$. Recall that

$$
P(\bar{B})=P(B) \text { and } P\left(\overline{B^{c}}\right)=P\left(B^{c}\right) \text { hold simultaneously } \Longleftrightarrow P(\partial B)=0,
$$

where $\partial B=\bar{B} \cap \overline{B^{c}}$ is the boundary of $B$. Thus, $B$ is $P$-continuous. It follows that $\lim _{n \rightarrow \infty} P_{n}(B)=P(B)$ since $P_{n} \rightarrow P$ weakly. Set

$$
A_{x}=\{\omega \in \Omega: \omega(I) \cap(F-x) \neq \emptyset\}, x \in \mathbb{R}^{d} .
$$

Then

$$
E\left\{\lambda_{d}\left[X^{n}(I)-F\right]\right\}=\int_{\mathbb{R}^{d}} P_{n}\left(A_{x}\right) d x, E\left\{\lambda_{d}[X(I)-F]\right\}=\int_{\mathbb{R}^{d}} P\left(A_{x}\right) d x .
$$

By Fatou's lemma,

$$
\liminf \int_{\mathbb{R}^{d}} P_{n}\left(A_{x}\right) d x \geq \int_{\mathbb{R}^{d}} \liminf P_{n}\left(A_{x}\right) d x .
$$

But we just showed that $\lim \inf P_{n}\left(A_{x}\right)=P\left(A_{x}\right)$ for each $x$. Thus, (4.41) is true.

Proposition 4.5. Let $\mathbf{x}_{t}=\mathbf{x}_{t_{1}}^{1}+\cdots+\mathbf{x}_{t_{N}}^{N}: \mathbb{R}^{N} \rightarrow \mathbb{R}^{d}$ be any symmetric additive Lévy process. Assume that each $\mathbf{x}^{j}$ has a strong Feller resolvent. Assume that $\tilde{\sigma}^{\alpha}$ is independent of $\mathbf{x}$. Then $X=\mathbf{x} \circ \tilde{\sigma}^{\alpha}$ is $\mathbf{b}$-related with respect to any $N$-dimensional compact interval $I \subset\left(\mathbb{R}_{2}^{N}\right)^{o}$ and $I^{\prime}$, where $X^{b}$ has stationary increments. More precisely, for any $N$-dimensional compact interval $I \subset\left(\mathbb{R}_{2}^{N}\right)^{o}$ and any compact $F \subset \mathbb{R}^{d}$

$$
E\left\{\lambda_{d}\left(\mathbf{x} \circ \tilde{\sigma}^{\alpha}(I)-F\right)\right\}>0 \Longrightarrow \int_{\mathbb{R}^{d}}|\hat{\mu}(\xi)|^{2} Q_{\kappa}^{\mathbf{x} \circ \sigma^{\alpha}}(\xi) d \xi<\infty \text { for some } \mu \in \mathcal{P}(F)
$$

Proof. The proof is long but contains some interesting ideas.

Step 1. For this kind of argument, one often starts with a representation of $\mathbf{x}$ as a stochastic integral. Consider an arbitrary symmetric two-sided Lévy process $U$ in $\mathbb{R}^{d}$ with Lévy exponent $\Psi$. (That is, if $V$ and $W$ are a pair of i.i.d. Lévy processes with Lévy exponent $\Psi$, define $U_{t}=V_{t}$ for $t \geq 0, U_{t}=W_{-t}$ for $t<0$, where $V$ is rcll while $W$ is lcrl.) Let $\tau: \mathbb{R}^{N} \rightarrow \mathbb{R}$ be any real-valued rcll field having $\mathbf{b}$, independent of $U$, and satisfying $\tau_{t_{1}} \leq \tau_{t_{2}} \leq \cdots \leq \tau_{t_{k}}$ for any increasing sequence $t_{1} \preceq t_{2} \preceq \cdots \preceq t_{k}, t_{i} \in \mathbb{R}^{N}$. $U$ has the following stochastic integral representation:

$$
U_{t}=\int_{\mathbb{R}} f(t, \lambda) \zeta(d \lambda), t \in \mathbb{R}
$$

where $f(t, \lambda)=1_{Q_{t}}(\lambda), Q_{t}=[0, t]$ if $t \geq 0$ but $Q_{t}=[t, 0]$ if $t<0$, and $\zeta$ is an $\mathbb{R}^{d_{-}}$ valued random measure on $\mathbb{R}$ with Lebesgue measure as the control measure and 
Lévy exponent $\Psi$, i.e., the one-parameter Lévy sheet associated with $\zeta$ is exactly $U$. For any fixed $h \in(0, \infty)$, we have an rcll process

$$
U_{t}^{h}=\int_{B_{h}} f(t, \lambda) \zeta(d \lambda), t \in \mathbb{R}
$$

where $B_{h}=[-h, h]$. Consider the rcll field $U^{h} \circ \tau: \mathbb{R}^{N} \rightarrow \mathbb{R}^{d}$. We will now show that $U^{h} \circ \tau$ has $\mathbf{b}$.

Let $\Omega_{1}$ be the sample space for $\tau$ and $\Omega_{2}$ for $U$. Fix some $l_{1} \geq h$. Let $\mathbf{b}_{2}$ be the $\mathbf{b}$ for $U$ with domain $\left[-l_{1}, l_{1}\right)$ such that $\mathbf{b}_{2}$ is independent of $U^{l_{1}}$ and $r_{2}\left(\mathbf{b}_{2}\left(\omega_{2}\right)\right)=$ $r_{2}\left(\omega_{2}\right)$ for each $\omega_{2} \in \Omega_{2}^{\prime} \subset \Omega_{2}$ with $r_{1}\left(\Omega_{2}^{\prime}\right)=\left(\Omega_{2}\right)_{l_{1}}$. (In this case $U$ is a "oneparameter iterated additive process" since $U$ is symmetric, or a "one-parameter Lévy sheet".) Similarly, let $\mathbf{b}_{1}$ be the $\mathbf{b}$ for $\tau$ on $\mathbb{R}^{N}$ such that $\mathbf{b}_{1}$ is independent of $\tau^{l}$ and $r_{1}\left(\mathbf{b}_{1}\left(\omega_{1}\right)\right)=r_{1}\left(\omega_{1}\right)$ for each $\omega_{1} \in \Omega_{1}^{\prime} \subset \Omega_{1}$ with $r_{1}\left(\Omega_{1}^{\prime}\right)=\left(\Omega_{1}\right)_{l}$.

Let

$$
A=\left\{\int_{B_{h}} f\left(\omega_{1}(t), \lambda\right) \zeta^{\omega_{2}}(d \lambda): \omega_{i} \in \Omega_{i}\right\} .
$$

Then $P(A)=1$, where $P$ is the law of $U^{h} \circ \tau$. The relation

$$
\int_{B_{h}} f\left(\omega_{1}(t), \lambda\right) \zeta^{\omega_{2}}(d \lambda)=\int_{B_{h}} f\left(\omega_{1}^{\prime}(t), \lambda\right) \zeta^{\omega_{2}^{\prime}}(d \lambda), \forall t, \omega_{i}, \omega_{i}^{\prime} \in \Omega_{i},
$$

divides $A$ into disjoint equivalence classes. Denote a class by $C$. Then there exists a well-defined measurable map $\mathbf{b}: A \rightarrow \Omega$ such that there is an $\int_{B_{h}} f\left(\omega_{1}(t), \lambda\right) \zeta^{\omega_{2}}(d \lambda)$ $\in C$ for each class $C$ and for any $\theta^{\prime}(t)=\int_{B_{h}} f\left(\omega_{1}^{\prime}(t), \lambda\right) \zeta^{\omega_{2}^{\prime}}(d \lambda) \in C$,

$$
\mathbf{b}\left(\theta^{\prime}\right)(t)=\int_{B_{h}} f\left(\mathbf{b}_{1}\left(\omega_{1}\right)(t), \lambda\right) \zeta^{\mathbf{b}_{2}\left(\omega_{2}\right)}(d \lambda),
$$

and if $\omega_{i}^{\prime} \in \Omega_{i}^{\prime}$ for all $i$, then $\omega_{i} \in \Omega_{i}^{\prime}$ for all $i$. Since $\mathbf{b}_{i}$ are independent and since $l_{1} \geq h, \mathbf{b}$ is independent of $\left(U^{h} \circ \tau\right)^{l}$. Set

$$
A^{\prime}=\left\{\int_{B_{h}} f\left(\omega_{1}(t), \lambda\right) \zeta^{\omega_{2}}(d \lambda): \omega_{i} \in \Omega_{i}^{\prime}\right\} .
$$

Clearly, $A^{\prime} \subset A$. Also $r\left(A^{\prime}\right)=r(A)$ because $r_{1}\left(\Omega_{1}^{\prime}\right)=\left(\Omega_{1}\right)_{l}$ and because $l_{1} \geq h$, which implies that $\int_{B_{h}} f\left(\omega_{1}(t), \lambda\right) \zeta^{\omega_{2}}(d \lambda)=\int_{B_{h}} f\left(\omega_{1}(t), \lambda\right) \zeta^{r_{2}\left(\omega_{2}\right)}(d \lambda)$. For each $\theta^{\prime} \in A^{\prime}, t \in J_{1}(l)$, where $J_{1}(l)$ is the domain for $\tau^{l}$, assuming $\theta^{\prime} \in C$, we have

$$
\begin{aligned}
& \mathbf{b}\left(\theta^{\prime}\right)(t)=\int_{B_{h}} f\left(\mathbf{b}_{1}\left(\omega_{1}\right)(t), \lambda\right) \zeta^{\mathbf{b}_{2}\left(\omega_{2}\right)}(d \lambda) \\
& =\int_{B_{h}} f\left(\omega_{1}(t), \lambda\right) \zeta^{\omega_{2}}(d \lambda)=\int_{B_{h}} f\left(\omega_{1}^{\prime}(t), \lambda\right) \zeta^{\omega_{2}^{\prime}}(d \lambda),
\end{aligned}
$$

i.e., $r\left(\mathbf{b}\left(\theta^{\prime}\right)\right)=r\left(\theta^{\prime}\right)$. The proof that $U^{h} \circ \tau$ has $\mathbf{b}$ on $\mathbb{R}^{N}$ is complete.

On the other hand, if we let $g(s, t, \xi, \lambda)=\Psi[(f(s, \lambda)-f(t, \lambda)) \xi]$ (recalling that $\Psi \geq 0)$, then

$E e^{i \xi \cdot\left(U_{s}-U_{t}\right)}=e^{-\int_{\mathbb{R}} g(s, t, \xi, \lambda) d \lambda}, E e^{i \xi \cdot\left(U_{s}^{h}-U_{t}^{h}\right)}=e^{-\int_{B_{h}} g(s, t, \xi, \lambda) d \lambda}, \forall s, t \in \mathbb{R}, \forall \xi \in \mathbb{R}^{d}$.

Thus,

$$
\begin{gathered}
E e^{i \xi \cdot\left(U \circ \tau_{s}-U \circ \tau_{t}\right)}=E e^{-\int_{\mathbb{R}} g\left(\tau_{s}, \tau_{t}, \xi, \lambda\right) d \lambda} \leq E e^{-\int_{B_{h}} g\left(\tau_{s}, \tau_{t}, \xi, \lambda\right) d \lambda} \\
=E e^{i \xi \cdot\left(U^{h} \circ \tau_{s}-U^{h} \circ \tau_{t}\right)}, \forall s, t \in \mathbb{R}^{N}, \forall \xi \in \mathbb{R}^{d} .
\end{gathered}
$$


Step 2. Let

$$
\mathbf{x}_{t}=\mathbf{x}_{t_{1}}^{1}+\cdots+\mathbf{x}_{t_{N}}^{N}, t \in \mathbb{R}^{N},
$$

be any symmetric additive Lévy process, where $\mathbf{x}^{j}$, a Lévy process, has Lévy expoenent $\Psi_{j}$. Let $\tilde{\sigma}^{j}$ be the $j$ th component of $\tilde{\sigma}^{\alpha}$, where $\mathbf{x}$ and $\tilde{\sigma}^{\alpha}$ are independent. Recall that $\tilde{\sigma}^{j}$ are i.i.d. real-valued additive Lévy processes. We have

$$
\mathbf{x} \circ \tilde{\sigma}_{t}^{\alpha}=\mathbf{x}^{1} \circ \tilde{\sigma}_{t}^{1}+\cdots+\mathbf{x}^{N} \circ \tilde{\sigma}_{t}^{N}, t \in \mathbb{R}^{N} .
$$

With the notation of Step 1, we define

$$
\mathbf{x}^{h} \circ \tilde{\sigma}_{t}^{\alpha}=\mathbf{x}^{1, h} \circ \tilde{\sigma}_{t}^{1}+\cdots+\mathbf{x}^{N, h} \circ \tilde{\sigma}_{t}^{N}, t \in \mathbb{R}^{N} .
$$

We just showed that every $\mathbf{x}^{j, h} \circ \tilde{\sigma}^{j}$ has $\mathbf{b}$ with domain $[-l, l)^{N}$ in Step 1. (Recall that $\tilde{\sigma}^{j}$ has $\mathbf{b}$ with domain $[-l, l)^{N}$ since $\tilde{\sigma}^{j}$ is an iterated additive process.) By Lemma 2.1 (cf. $Z^{3}$ ), $\mathbf{x}^{h} \circ \tilde{\sigma}^{\alpha}$ has $\mathbf{b}$ with domain $[-l, l)^{N}$ since the $\mathbf{x}^{j, h} \circ \tilde{\sigma}^{j}$ are independent.

It is quite clear that there is a sequence $h_{n} \uparrow \infty$ such that $\mathbf{x}^{h_{n}} \circ \tilde{\sigma}^{\alpha} \stackrel{\text { law }}{\longrightarrow} \mathbf{x} \circ \tilde{\sigma}^{\alpha}$. Since each symmetric $\mathbf{x}^{j}$ has a strong Feller resolvent, $\left(\mathbf{x}_{t_{1}}, \cdots, \mathbf{x}_{t_{p}}\right)$ has a joint density for any distinct $t_{1}, \cdots, t_{p} \in \mathbb{R}^{N} \backslash\{0\}$. It follows from Lemma 4.3 that $\mathbf{x} \circ \tilde{\sigma}^{\alpha}$ is an $M$-field on any $N$-dimensional compact interval $J \subset\left(\mathbb{R}_{2}^{N}\right)^{o}$. Denote $\mathbf{x}^{h_{n}} \circ \tilde{\sigma}^{\alpha}$ by $W^{n}$. Fix a compact $F \subset \mathbb{R}^{d}$ and assume that $E\left\{\lambda_{d}(X(I)-F)\right\}>0$, where $I \subset\left(\mathbb{R}_{2}^{N}\right)^{o}$ is an $N$-dimensional compact interval. By Lemma 4.4, there is $W^{n}$ for some large $n$ such that $E\left\{\lambda_{d}\left(W^{n}(I)-F\right)\right\}>0$. It is easy to see that $\mathbf{x}^{j, h}$ has stationary increments (but no longer has independent increments). Thus, $W^{n}$ has stationary increments. (Here $W^{n}$ serves as $X^{b}$, which depends on $I$ and $F$.) On the other hand, (4.46) shows that $Q_{\eta}^{\mathbf{x} \circ \tilde{\sigma}^{\alpha}} \leq Q_{\eta}^{W^{n}}$ for any $\eta \in \mathcal{P}\left(\mathbb{R}^{N}\right)$. The assertion that $X=\mathbf{x} \circ \tilde{\sigma}^{\alpha}$ is b-related with respect to any $N$-dimensional compact interval $I \subset\left(\mathbb{R}_{2}^{N}\right)^{o}$ is now proved. Since $W^{n}$ has $\mathbf{b}$ with domain $[-l, l)^{N}$, part (i) of Proposition 4.1 implies that

$$
E\left\{\lambda_{d}\left(W^{n}(I)-F\right)\right\}>0 \Longrightarrow E\left\{\lambda_{d}\left(W^{n}\left(\mathbb{R}_{2}^{N}\right)-F\right)\right\}>0 \Longrightarrow|\hat{\mu}(\xi)|^{2} Q_{\kappa^{2}}^{W^{n}}(\xi) \in L^{1}
$$

for some $\mu \in \mathcal{P}(F)$. Here is a technical fact. One can show that if $Y: \mathbb{R}^{N} \rightarrow \mathbb{R}^{d}$ is an rcll field with stationary increments, then $Q_{\kappa^{2}}^{Y \circ \tilde{\sigma}^{\alpha}}=Q_{\kappa}^{Y \circ \sigma^{\alpha}}$. Now $W^{n}=\mathbf{x}^{h_{n}} \circ \tilde{\sigma}^{\alpha}$, where $\mathbf{x}_{t}^{h_{n}}=\mathbf{x}_{t_{1}}^{1, h_{n}}+\cdots+\mathbf{x}_{t_{N}}^{N, h_{n}}$ has stationary increments. We can conclude that

$$
|\hat{\mu}(\xi)|^{2} Q_{\kappa}^{W^{n}}(\xi) \in L^{1} .
$$

But (4.46) shows that $Q_{\kappa}^{\mathbf{x} \circ \sigma^{\alpha}} \leq Q_{\kappa}^{W^{n}}$. Thus, $|\hat{\mu}(\xi)|^{2} Q_{\kappa}^{\mathbf{x} \circ \sigma^{\alpha}}(\xi) \in L^{1}$.

Additive sheets and fractional fields. An additive sheet is an $\left(\mathbb{R}^{N} \times \Omega\right)$-measurable $\mathbb{R}^{d}$-valued random set function $\zeta^{\omega}(B), B \in \mathcal{B}\left(\mathbb{R}^{N}\right)$ such that $\zeta\left(B_{1}\right), \cdots, \zeta\left(B_{k}\right), k=$ $2,3, \cdots$, are independent whenever $B_{1}, \cdots, B_{k}$, are disjoint. Write $\mathbb{R}_{o}^{N}$ for the disjoint union of all open quadrants $\left(2^{N}\right.$ total $)$ of $\mathbb{R}^{N}$. Let $t=\left(t_{1}, \cdots, t_{N}\right) \in$ $\mathbb{R}_{o}^{N}$. Then the nonzero coordinates $t_{1}, \cdots, t_{N}$ determine an $N$-dimensional compact interval $Q_{t}$ of $\mathbb{R}^{N}$ with two special vertices at 0 and $t$. (If $t \in(0, \infty)^{N}, Q_{t}$ is usually denoted by $[0, t]=\left[0, t_{1}\right] \times \cdots \times\left[0, t_{N}\right]$.) The field $X_{t}=\zeta\left(Q_{t}\right), t \in \mathbb{R}_{o}^{N}$, and $X_{t}=0, t \in \mathbb{R}^{N} \backslash \mathbb{R}_{o}^{N}$, is also called the additive sheet. (In some circumstances, one considers only the field $X_{t}=\zeta([0, t]), t \in(0, \infty)^{N}$.) It is well known that any additive sheet $X$ has a version in which a.s. the path of $X$ is rcll in the usual partial order, and $X$ is continuous in probability. If $E e^{i \xi \cdot \zeta(B)}=e^{-\lambda_{N}(B) \Psi(\xi)}, \xi \in \mathbb{R}^{d}, B \in$ $\mathcal{B}\left(\mathbb{R}^{N}\right)$, where $\Psi(\xi)$ is a Lévy exponent, then $\zeta$ is called the Lévy sheet. If $\Psi$ is real-valued, $\zeta$ is called a symmetric Lévy sheet. Let $X$ be an additive sheet. Given 
any point $t_{0} \in \mathbb{R}^{N}$, we make a coordinate system translation so that the origin of the new coordinate system is at $t_{0}$. Since disjoint increments over intervals are independent, along the $2^{N}$ half-axes of the new coordinate system, $X_{t}-X_{t_{0}}$ gives rise to $2^{N}$ independent additive processes. This has two important consequences: (a) for each $i \in\{1, \cdots, N\}$, the one-parameter increment field $X_{\left(\cdot, t^{i}+l, \cdot\right)}-X_{(\cdot, l, \cdot)}$, $t^{i} \geq 0$, is independent of $X_{s}$ for all $s \in \mathbb{R}^{i-1} \times(-\infty, l] \times \mathbb{R}^{N-i}$. (b) $X$ has independent increments in any partial order of $\mathbb{R}^{N}$. If $X$ is a symmetric Lévy sheet, then the additive processes are Lévy processes.

Lemma 4.6. Any additive sheet $X$ has $\mathbf{b}$.

Proof. Assume that $X$ is in the rcll version and in the canonical form, i.e., $X_{t}(\omega)=$ $\omega(t)$. We will write $\omega\left(t^{1}, \cdot\right)$ for $\omega(t), t=\left(t^{1}, \cdots, t^{N}\right)$. Fix $l \in(0, \infty)$. Let $A=\{\omega \in$ $\left.\Omega: \omega\left(\mathbb{R}^{N} \backslash \mathbb{R}_{o}^{N}\right)=0\right\}$. By definition of the additive sheet, $P(A)=1$. Let $\Omega_{+}$be the space of rcll functions from $\mathbb{R}_{+} \times \mathbb{R}^{N-1}$ into $\mathbb{R}^{d}$. Define a measurable mapping $\mathbf{b}_{A}^{+}: A \rightarrow \Omega_{+}$by $\mathbf{b}_{A}^{+}(\omega)(t)=\omega\left(t^{1}+l, \cdot\right)-\omega(l, \cdot), t \in \mathbb{R}_{+} \times \mathbb{R}^{N-1}$, for $\omega \in A$. Since $\zeta$ is an additive sheet, $\mathbf{b}_{A}^{+}$is independent of $X^{l}$. Let $\Omega_{-}$be the space of rcll functions from $(-\infty, 0) \times \mathbb{R}^{N-1}$ into $\mathbb{R}^{d}$. Define a measurable mapping $\mathbf{b}_{A}^{-}: A \rightarrow \Omega_{-}$by $\mathbf{b}_{A}^{-}(\omega)(t)=\omega\left(t^{1}-l, \cdot\right)-\omega\left((-l)_{-}, \cdot\right), t \in(-\infty, 0) \times \mathbb{R}^{N-1}$, for $\omega \in A$. Since $\zeta$ is an additive sheet, $\mathbf{b}_{A}^{-}$is independent of $X^{l}$ as well. Define a measurable mapping $\mathbf{b}_{A}: A \rightarrow \Omega$ by $\mathbf{b}_{A}(\omega)\left(t^{1}, \cdot\right)=\mathbf{b}_{A}^{+}(\omega)\left(t^{1}, \cdot\right)$ for $t^{1} \geq 0$ and $\mathbf{b}_{A}(\omega)\left(t^{1}, \cdot\right)=\mathbf{b}_{A}^{-}(\omega)\left(t^{1}, \cdot\right)$ for $t^{1}<0$, for each $\omega \in A$. Since $\mathbf{b}_{A}^{+}$and $\mathbf{b}_{A}^{-}$are independent, $\mathbf{b}_{A}$ is independent of $X^{l}$.

Fix any $\omega \in r(A)$. We will extend $\omega$ to some $\omega^{\prime} \in A$ as follows. Let $\omega^{\prime}\left(t^{1}, \cdot\right)=$ $\omega\left(t^{1}, \cdot\right), t^{1} \in[0, l)$, and $\omega^{\prime}\left(t^{1}+l, \cdot\right)=\omega\left(t^{1}, \cdot\right), t^{1} \in[0, l) \backslash\{0\}$, which implies that $\omega^{\prime}(l, \cdot)=\omega(0, \cdot)=0$ by the right-continuity and the boundary condition for sheets. Let $\omega^{\prime}\left(t^{1}, \cdot\right)=\omega\left(t^{1}, \cdot\right), t^{1} \in[-l, 0)$, and $\omega^{\prime}\left(t^{1}-l, \cdot\right)=\omega\left(t^{1}, \cdot\right), t^{1} \in[-l, 0)$, which implies that $\omega^{\prime}\left((-l)_{-}, \cdot\right)=\omega\left(0_{-}, \cdot\right)=0$ since $\omega$ has left limits and satisfies the boundary condition for sheets. For $s \in \mathbb{R}^{N} \backslash\left\{t: t^{1} \in[-2 l, 2 l)\right\}, \omega^{\prime}(s)$ can be defined in any possible way as long as $\omega^{\prime}$ is rcll on $\mathbb{R}^{N}$ and $\omega^{\prime} \in A$, and ultimately some event $A^{\prime} \subset A$ is achieved after all $\omega \in r(A)$ are used. Obviously, $r\left(A^{\prime}\right)=r(A)$. For each $\omega^{\prime} \in A^{\prime}$, we have

$$
\begin{aligned}
\mathbf{b}_{A}\left(\omega^{\prime}\right)\left(t^{1}, \cdot\right) & =\mathbf{b}_{A}^{+}\left(\omega^{\prime}\right)\left(t^{1}, \cdot\right)=\omega^{\prime}\left(t^{1}+l, \cdot\right)-\omega^{\prime}(l, \cdot)=\omega\left(t^{1}, \cdot\right)-0=\omega\left(t^{1}, \cdot\right) \\
& =\omega^{\prime}\left(t^{1}, \cdot\right), t^{1} \in[0, l) \\
\mathbf{b}_{A}\left(\omega^{\prime}\right)\left(t^{1}, \cdot\right) & =\mathbf{b}_{A}^{-}\left(\omega^{\prime}\right)\left(t^{1}, \cdot\right)=\omega^{\prime}\left(t^{1}-l, \cdot\right)-\omega^{\prime}\left((-l)_{-}, \cdot\right)=\omega\left(t^{1}, \cdot\right)-0=\omega\left(t^{1}, \cdot\right) \\
& =\omega^{\prime}\left(t^{1}, \cdot\right), t^{1} \in[-l, 0) .
\end{aligned}
$$

That is, $r\left[\mathbf{b}_{A}\left(\omega^{\prime}\right)\right]=r\left(\omega^{\prime}\right)$ for each $\omega^{\prime} \in A^{\prime}$. The proof that $X$ has $\mathbf{b}$ is complete.

Since $X$ is an additive sheet, it is quite clear that in the above argument if we construct b over any $\mathbb{R}^{m_{1}} \times[-l, l] \times \mathbb{R}^{m_{2}}$, where $m_{1}+m_{2}=N-1$, instead of $[-l, l) \times \mathbb{R}^{N-1}$, the result remains unchanged.

We can now define the Wiener integral with respect to an $\mathbb{R}^{d}$-valued random measure on $\mathbb{R}^{N}$ and the so-called linear fractional field. Let $\zeta(B), B \in \mathcal{B}\left(\mathbb{R}^{N}\right)$, be an $\mathbb{R}^{d}$-valued random measure, i.e., an $\mathbb{R}^{d}$-valued additive sheet $\zeta$ on $\mathbb{R}^{N}$, such that $E e^{i \xi \cdot \zeta(B)}=e^{-m(B) \Psi(\xi)}$, where $B \in \mathcal{B}\left(\mathbb{R}^{N}\right), \xi \in \mathbb{R}^{d}, \Psi(\xi)$ is a Lévy exponent and $m$, called the control measure, is a measure on $\mathbb{R}^{N}$ such that $m(K)<\infty$ for 
all compacts $K$. Let $f: \mathbb{R}^{N} \rightarrow \mathbb{R}$ be a measurable function such that $\Psi(f(s) \xi) \in$ $L^{1}(m)$ for each $\xi \in \mathbb{R}^{d}$. We can define the $\mathbb{R}^{d}$-valued Wiener stochastic integral $\int_{\mathbb{R}^{N}} f(s) \zeta(d s)$ with the characteristic function

$$
E e^{i \xi \cdot \int_{\mathbb{R}^{N}} f(s) \zeta(d s)}=e^{-\int_{\mathbb{R}^{N}} \Psi(f(s) \xi) m(d s)}, \xi \in \mathbb{R}^{d} .
$$

These integrals driven by a common random measure $\zeta$ have the following joint distribution. By a.s. linearity of integration,

$$
E e^{i \sum_{k=1}^{p} \xi_{k} \cdot \int_{\mathbb{R}^{N}} f_{k}(s) \zeta(d s)}=\exp \left\{-\int_{\mathbb{R}^{N}} \Psi\left(\sum_{k=1}^{p} \xi_{k}^{1} f_{k}(\lambda), \cdots, \sum_{k=1}^{p} \xi_{k}^{d} f_{k}(\lambda)\right) m(d \lambda)\right\},
$$

where $\xi_{1}, \cdots, \xi_{p} \in \mathbb{R}^{d}, t_{1}, \cdots, t_{p} \in \mathbb{R}^{N}, \xi_{k}=\left(\xi_{k}^{1}, \cdots, \xi_{k}^{d}\right)$, and $p$ is any integer. These properties can be used to define the following random field in $\mathbb{R}^{d}$, called the linear fractional field:

$$
X_{t}=\int_{\mathbb{R}^{N}} f(t, s) \zeta(d s), t \in \mathbb{R}^{N},
$$

where $f: \mathbb{R}^{N} \times \mathbb{R}^{N} \rightarrow \mathbb{R}$ is a measurable function such that

$$
\Psi\left(\sum_{k=1}^{p} \xi_{k}^{1} f\left(t_{k}, \lambda\right), \cdots, \sum_{k=1}^{p} \xi_{k}^{d} f\left(t_{k}, \lambda\right)\right) \in L^{1}(m)
$$

for all $\xi_{1}, \cdots, \xi_{p} \in \mathbb{R}^{d}, t_{1}, \cdots, t_{p} \in \mathbb{R}^{N}$, and integer $p$. $X$ is called fractional stable if $\Psi$ is stable.

The harmonizable fractional field is defined by the Wiener integral against a $\mathbb{C}^{d}$-valued random measure. Let $\zeta$ be a $\mathbb{C}^{d}$-valued additive sheet, i.e., an $\mathbb{R}^{2 d_{-}}$ valued additive sheet. Note that $\operatorname{Re} \zeta$ and $\operatorname{Im} \zeta$ are both $\mathbb{R}^{d}$-valued additive sheets. Furthermore, for each $c \in \mathbb{C}, c \zeta$ is also a $\mathbb{C}^{d}$-valued additive sheet. $\zeta$ is called a $\mathbb{C}^{d}$-valued random measure if there exist a measure $m$ on $\mathbb{R}^{N}$ satisfying $m(K)<\infty$ for all compacts $K$, known as the control measure, and two Lévy exponents $\Psi_{r}(c, \cdot)$ and $\Psi_{i}(c, \cdot)$ for each $c \in \mathbb{C}$ such that

$$
\begin{aligned}
& E e^{i \xi \cdot \operatorname{Re}(c \zeta(A)-\overline{c \zeta(-B)})}=e^{-m(A \triangle B) \Psi_{r}(c, \xi)}, \xi \in \mathbb{R}^{d}, A, B \in \mathcal{B}\left(\mathbb{R}^{N}\right), \\
& E e^{i \xi \cdot \operatorname{Im}(c \zeta(A)-\overline{c \zeta(-B)})}=e^{-m(A \triangle B) \Psi_{i}(c, \xi)}, \xi \in \mathbb{R}^{d}, A, B \in \mathcal{B}\left(\mathbb{R}^{N}\right),
\end{aligned}
$$

where $\triangle$ denotes the symmetric difference. We have for example,

$$
E e^{i \xi \cdot[(\operatorname{Re} c) \operatorname{Re} \zeta(A)-(\operatorname{Im} c) \operatorname{Im} \zeta(A)]}=e^{-m(A) \Psi_{r}(c, \xi)}, \xi \in \mathbb{R}^{d}, A \in \mathcal{B}\left(\mathbb{R}^{N}\right) .
$$

Let $f: \mathbb{R}^{N} \rightarrow \mathbb{C}$ satisfy $\Psi_{r}(f(t), \xi) \in L^{1}(m)$ and $\Psi_{i}(f(t), \xi) \in L^{1}(m)$ for each $\xi \in \mathbb{R}^{d}$. We can define the $\mathbb{C}^{d}$-valued Wiener stochastic integral $\int_{\mathbb{R}^{N}} f(t) \zeta(d t)$ such that

$$
\begin{gathered}
E e^{i \xi \cdot \operatorname{Re} \int_{\mathbb{R}^{N}} f(t) \zeta(d t)}=e^{-\int_{\mathbb{R}^{N}} \Psi_{r}(f(t), \xi) m(d t)}, \\
E e^{i \xi \cdot \operatorname{Im} \int_{\mathbb{R}^{N}} f(t) \zeta(d t)}=e^{-\int_{\mathbb{R}^{N}} \Psi_{i}(f(t), \xi) m(d t)}, \xi \in \mathbb{R}^{d} .
\end{gathered}
$$

Furthermore, $\int f$ satisfies $\int(a f+b g)=a \int f+b \int g$ a.s. for $a, b \in \mathbb{R}$, and if $d=1$,

$$
E e^{i \sum_{j=1}^{p} \xi_{j} \operatorname{Re} \int_{\mathbb{R}^{N}} f_{j}(t) \zeta(d t)}=e^{-\int_{\mathbb{R}^{N}} \Psi_{r}\left(\sum_{j=1}^{p} \xi_{j} f_{j}(t), 1\right) m(d t)}, \xi_{j} \in \mathbb{R} .
$$

A $\mathbb{C}^{d}$-valued random measure $\zeta$ is not a simple $\mathbb{R}^{2 d}$-valued additive sheet. It has its own unique structure. This makes $\mathbb{C}^{d}$-valued random measures technically demanding. (1) Write $f=f_{r}+i f_{i}, \quad \zeta=\zeta_{r}+i \zeta_{i}$. Then for $X=\int_{\mathbb{R}^{N}} f(\lambda) \zeta(d \lambda)$, 
$\operatorname{Re} X=\int_{\mathbb{R}^{N}} f_{r}(\lambda) \zeta_{r}(d \lambda)-\int_{\mathbb{R}^{N}} f_{i}(\lambda) \zeta_{i}(d \lambda)$. (2) Set $B=\emptyset$ in (H1) and (H2). Then for each $c \in \mathbb{C}, E e^{i \xi \cdot \operatorname{Rec} \zeta(A)}=e^{-m(A) \Psi_{r}(c, \xi)}, E e^{i \xi \cdot \operatorname{Im} c \zeta(A)}=e^{-m(A) \Psi_{i}(c, \xi)}$, i.e., both $\operatorname{Re} c \zeta$ and $\operatorname{Im} c \zeta$ are $\mathbb{R}^{d}$-valued random measures. In particular, $E e^{i \xi \cdot \operatorname{Re} \zeta(A)}=$ $e^{-m(A) \Psi_{r}(1, \xi)}, E e^{i \xi \cdot \operatorname{Im} \zeta(A)}=e^{-m(A) \Psi_{i}(1, \xi)}$. (3) Take $A=B$ and $c=1$ in (H1). We obtain $\operatorname{Re} \zeta(A)=\operatorname{Re} \overline{\zeta(-A)}=\operatorname{Re} \zeta(-A)$ a.s. Similarly, we have $\operatorname{Imc} \zeta(B)=$ $-\operatorname{Im} c \zeta(-B)$ a.s. for each $c \in \mathbb{C}$. Note that

$\operatorname{Re} \zeta(A)=\operatorname{Re} \zeta(-A) a . s . \Longrightarrow m$ is a symmetric Radon measure (i.e., $m(-A)=m(A)$ ), e.g., $\lambda_{N},\left.\lambda_{1}\right|_{\left[-a^{\prime}, a^{\prime}\right]}, a^{\prime} \in(0, \infty)$, etc. Similarly,

$$
\operatorname{Im} c \zeta(B)=-\operatorname{Im} c \zeta(-B) \text { a.s. } \Longrightarrow \Psi_{i}(c, \xi)=\Psi_{i}(c,-\xi),
$$

i.e., each $\Psi_{i}(c, \xi)$ is a symmetric Lévy exponent, i.e., $\Psi_{i}(c, \xi) \geq 0$. Thus, the $\mathbb{R}^{d}$-valued random measure $\operatorname{Im} c \zeta$ is a symmetric random measure.

The $\mathbb{R}^{d}$-valued field

$$
X_{t}=\operatorname{Re} \int_{\mathbb{R}^{N}} f(t, \lambda) \zeta(d \lambda), t \in \mathbb{R}^{N},
$$

where $f: \mathbb{R}^{N} \times \mathbb{R}^{N} \rightarrow \mathbb{C}$ is a measurable function such that $\Psi_{r}(f(t, \lambda), \xi) \in L^{1}(m)$ for each $t \in \mathbb{R}^{N}$ and each $\xi \in \mathbb{R}^{d}$, is called the harmonizable fractional field.

Let $\zeta$ be a symmetric $\mathbb{C}^{d}$-valued $\alpha$-stable random measure with a control measure $m$. When $\alpha=2, \zeta$ is called a $\mathbb{C}^{d}$-valued Gaussian random measure with control measure $m$. More precisely, in this paper we consider only the case where $\zeta$ is essentially an isotropic $\alpha$-stable random measure in $\mathbb{R}^{2 d}$. (For other $\mathbb{C}^{d}$-valued random measures $\zeta$, it is difficult to characterize the joint distributions.) So, let $\zeta=\left(m, a\|\xi\|^{\alpha}\right)$, where $\alpha \in(0,2], a \in(0, \infty)$ some constant, and $\xi \in \mathbb{R}^{2 d}$. $\zeta$ is called the $\mathbb{C}^{d}$-valued isotropic $\alpha$-stable random measure on $\mathbb{R}^{N}$ with control measure $m$. Another case is where the complex-valued components of $\zeta$ are independent, i.e., $\zeta=\left(m, \sum_{i=1}^{d} a_{i}\left\|\xi^{i}\right\|^{\alpha}\right)$, where $a_{i} \in(0, \infty), \xi^{i} \in \mathbb{R}^{2}$. Then either $\Psi_{r}(c, \xi)=b\|\xi\|^{\alpha}|c|^{\alpha}, \xi \in \mathbb{R}^{d}, c \in \mathbb{C}$, where $b \in(0, \infty)$ is some constant, or $\Psi_{r}(c, \xi)=b|c|^{\alpha}\left(a_{1}\left|\xi^{1}\right|^{\alpha}+\cdots+a_{d}\left|\xi^{d}\right|^{\alpha}\right), \xi=\left(\xi^{1} \cdots, \xi^{d}\right) \in \mathbb{R}^{d}, c \in \mathbb{C}$. Let $f(t, \lambda): \mathbb{R}^{2 N} \rightarrow \mathbb{C}$ be a $\mathbb{C}$-valued function satisfying $|f(t, \cdot)|^{\alpha} \in L^{1}(m)$ for each $t$, which gives rise to an $\mathbb{R}^{d}$-valued symmetric $\alpha$-stable field (called the harmonizable fractional $\alpha$-stable field)

$$
X_{t}=\operatorname{Re} \int_{\mathbb{R}^{N}} f(t, \lambda) \zeta(d \lambda)
$$

with

$$
E e^{i \xi \cdot\left(X_{t}-X_{s}\right)}=e^{-b\|\xi\|^{\alpha} \int_{\mathbb{R}^{N}}|f(t, \lambda)-f(s, \lambda)|^{\alpha} m(d \lambda)}, t, s \in \mathbb{R}^{N}, \xi \in \mathbb{R}^{d}
$$

or

$$
\begin{aligned}
& E e^{i \xi \cdot\left(X_{t}-X_{s}\right)}=e^{-b\left(\sum_{j=1}^{d} a_{j}\left|\xi^{j}\right|^{\alpha}\right) \int_{\mathbb{R}^{N}}|f(t, \lambda)-f(s, \lambda)|^{\alpha} m(d \lambda)}, \\
& t, s \in \mathbb{R}^{N}, \xi=\left(\xi^{1} \cdots, \xi^{d}\right) \in \mathbb{R}^{d},
\end{aligned}
$$

in which case the components of $X$ are independent.

Let $X: \mathbb{R}^{N} \rightarrow \mathbb{R}^{d}$ be any rcll fractional field. Assume that $X$ has a control measure $m$ whose support is contained in $\mathbb{R}^{m_{1}} \times[a, b] \times \mathbb{R}^{m_{2}}$, where $m_{1}+m_{2}=$ $N-1,-\infty<a<b<\infty$. If this holds, $m$ will be said to satisfy condition (CS). Consider fields of these mixture types: $Z_{t}^{1}=\left(X_{t}^{1}, \cdots, X_{t}^{n}\right), Z_{t}^{2}=\left(X_{t_{1}}^{1}, \cdots, X_{t_{n}}^{n}\right)$, 
$t=\left(t_{1}, \cdots, t_{n}\right), Z_{t}^{3}=X_{t}^{1}+\cdots+X_{t}^{n}$, and $Z_{t}^{4}=X_{t_{1}}^{1}+\cdots+X_{t_{n}}^{n}, t=\left(t_{1}, \cdots, t_{n}\right)$, where $X^{i}: \mathbb{R}^{N_{i}} \rightarrow \mathbb{R}^{d_{i}}$ are independent fractional fields, each of which has a control measure satisfying (CS).

Lemma 4.7. Let $X: \mathbb{R}^{N} \rightarrow \mathbb{R}^{d}$ be one of $Z^{1}, Z^{2}, Z^{3}$ and $Z^{4}$ as above. Then $X$ has $\mathbf{b}$.

Proof. Let $X: \mathbb{R}^{N} \rightarrow \mathbb{R}^{d}$ be any rcll fractional field. Assume that $X$ has a control measure $m$ which satisfies condition (CS). By Lemma 2.1, it suffices to show that $X$ has b. First assume that $X$ is linear and write

$$
X_{t}=\int_{\mathbb{R}^{N_{1}} \times[a, b] \times \mathbb{R}^{N_{2}}} f(t, s) \zeta(d s), t \in \mathbb{R}^{N} .
$$

Let $Y$ be the additive sheet associated with $\zeta$. (4.47) implies that $X$ is adapted to $Y$ with respect to the domain $\mathbb{R}^{N_{1}} \times[-l, l] \times \mathbb{R}^{N_{2}}$ for $l \geq|a| \vee|b|$. By Lemma 4.6, $Y$ has $\mathbf{b}$ with respect to the domain $\mathbb{R}^{N_{1}} \times[-l, l] \times \mathbb{R}^{N_{2}}$. It follows that $X$ has $\mathbf{b}$ as well.

Now assume that $X$ is harmonizable and let

$$
X_{t}=\operatorname{Re} \int_{\mathbb{R}^{N_{1}} \times[a, b] \times \mathbb{R}^{N_{2}}} f(t, s) \zeta(d s), t \in \mathbb{R}^{N} .
$$

Consider the $\mathbb{R}^{2 d}$-valued field

$$
H_{t}=\int_{\mathbb{R}^{N_{1}} \times[a, b] \times \mathbb{R}^{N_{2}}} f(t, s) \zeta(d s), t \in \mathbb{R}^{N},
$$

where $\zeta$ is an $\mathbb{R}^{2 d}$-valued additive sheet. Let $Y$ be the $\mathbb{R}^{2 d}$-valued additive sheet associated with $\zeta$. Since $H$ is adapted to $Y$ as in the linear case, $H$ has $\mathbf{b}$, which is the very same $\mathbf{b}$ for $Y$ (or $\zeta)$. Write $H_{t}=\left(X_{t}, \operatorname{Im} H_{t}\right)$. By Lemma $2.1\left(\right.$ cf. $\left.Z^{5}\right)$, both $X$ and $\operatorname{Im} H$ have $\mathbf{b}$ because $H$ does.

Interestingly, strictly $\alpha$-stable fractional fields usually have control measures satisfying (CS), which implies that these fractional fields have b by Lemma 4.7.

Proposition 4.8. Let $X: \mathbb{R}^{N} \rightarrow \mathbb{R}^{d}$ be an rcll fractional $\alpha$-stable field. Assume that if $X$ is linear, then $\Psi$ is strictly stable, and if $X$ is harmonizable, then $d=1$ and the complex-valued random measure is isotropic. Let $m$ be a control measure of $X$. Assume that $m$ takes the form $m=m_{1} \otimes h(x) d x \otimes m_{2}$, where $m_{1}$ is some Radon measure on $\mathbb{R}^{N_{1}}, m_{2}$ is some Radon measure on $\mathbb{R}^{N_{2}}, N_{1}+N_{2}=N-1$, and $h: \mathbb{R} \rightarrow(0, \infty)$ is some measurable function. Then $X$ has an integral representation such as

$$
X_{t}=\int_{\mathbb{R}^{N_{1}} \times[a, b] \times \mathbb{R}^{N_{2}}} F(t, s) \zeta_{1}(d s), t \in \mathbb{R}^{N},
$$

where $\zeta_{1}=(n, \Psi)$ is an $\alpha$-stable random measure with control measure $n=m_{1} \otimes$ $\left.\lambda_{1}\right|_{[a, b]} \otimes m_{2}$, where $\left.\lambda_{1}\right|_{[a, b]}$ is the restriction of $\lambda_{1}$ to $[a, b]$, and

$$
X_{t}=\operatorname{Re} \int_{\mathbb{R}^{N_{1}} \times[a, b] \times \mathbb{R}^{N_{2}}} F(t, s) \zeta_{1}(d s), t \in \mathbb{R}^{N},
$$

in the harmonizable case, where $\zeta_{1}=(n, \Psi)\left(\Psi(\xi)=a^{\prime}\|\xi\|^{\alpha}, \xi \in \mathbb{R}^{2}, a^{\prime} \in(0, \infty)\right)$ is a complex-valued isotropic $\alpha$-stable random measure with control measure $n=$ $\left.m_{1} \otimes \lambda_{1}\right|_{[-b, b]} \otimes m_{2}$, for some $b \in(0, \infty)$. 
Proof. First assume that $X$ is linear. Write

$$
X_{t}=\int_{\mathbb{R}^{N}} f(t, s) \zeta(d s), t \in \mathbb{R}^{N},
$$

and $\zeta=(m, \Psi)$, where $\Psi$ is strictly $\alpha$-stable. Recall that a Lévy exponent $\Psi$ is called (nontrivial) strictly $\alpha$-stable if $c \Psi(\xi)=\Psi\left(c^{1 / \alpha} \xi\right)$ for all $c>0$ and $\xi \in \mathbb{R}^{d}$. (A special case: $\alpha=1$. In this case $\Psi$ is strictly 1-stable iff $\int_{\mathbb{R}^{d}} \xi \mu(d \xi)=0$, where $\mu \in \mathcal{P}\left(S^{d-1}\right), S^{d-1}=\left\{\xi \in \mathbb{R}^{d}:\|\xi\|=1\right\}$, is the defining measure for $\Psi$.) The law of $X$ is given by

$$
E e^{i \sum_{k=1}^{p} \xi_{k} \cdot X_{t_{k}}}=\exp \left\{-\int_{\mathbb{R}^{N}} \Psi\left(\sum_{k=1}^{p} \xi_{k}^{1} f\left(t_{k}, \lambda\right), \cdots, \sum_{k=1}^{p} \xi_{k}^{d} f\left(t_{k}, \lambda\right)\right) m(d \lambda)\right\} .
$$

For $-\infty<a<b<\infty$, let $g$ be a smooth bijective increasing mapping from $(a, b)$ onto $\mathbb{R}$ such that $g(a+)=-\infty$ and $g(b-)=+\infty$, e.g., $g(x)=\tan x, x \in$ $(-\pi / 2, \pi / 2), a=-\pi / 2$, and $b=\pi / 2$, so that $g^{\prime}>0$. Since $\Psi$ is strictly $\alpha$-stable,

$$
\begin{aligned}
& E e^{i \sum_{k=1}^{p} \xi_{k} \cdot X_{t_{k}}}=\exp \left\{-\int_{\mathbb{R}^{N}} \Psi\left(\sum_{k=1}^{p} \xi_{k}^{1} f\left(t_{k}, \lambda\right), \cdots, \sum_{k=1}^{p} \xi_{k}^{d} f\left(t_{k}, \lambda\right)\right) m(d \lambda)\right\} \\
& =\exp \left\{-\int_{\mathbb{R}^{N_{1}}} \int_{a}^{b} \int_{\mathbb{R}^{N_{2}}} \Psi\left(\sum_{k=1}^{p} \xi_{k}^{1} f\left[t_{k},(u, g(x), v)\right] h(g(x))^{1 / \alpha} g^{\prime}(x)^{1 / \alpha}, \cdots,\right.\right. \\
& \left.\left.\quad \sum_{k=1}^{p} \xi_{k}^{d} f\left[t_{k},(u, g(x), v)\right] h(g(x))^{1 / \alpha} g^{\prime}(x)^{1 / \alpha}\right) m_{1}(d u) d x m_{2}(d v)\right\} .
\end{aligned}
$$

Thus, if we let $F[t,(u, x, v)]=f[t,(u, g(x), v)] h(g(x))^{1 / \alpha} g^{\prime}(x)^{1 / \alpha}, x \in(a, b)$, and $F[t,(u, x, v)]=0$ if $x \notin(a, b)$, then by (4.52) and (4.53) which show that two fractional fields have identical law, we get a version of $X$ given by

$$
X_{t}=\int_{\mathbb{R}^{N_{1}} \times[a, b] \times \mathbb{R}^{N_{2}}} F(t, s) \zeta_{1}(d s), t \in \mathbb{R}^{N},
$$

where $\zeta_{1}=(n, \Psi)$ is an $\alpha$-stable random measure with control measure $n=m_{1} \otimes$ $\left.\lambda_{1}\right|_{[a, b]} \otimes m_{2}$, where $\left.\lambda_{1}\right|_{[a, b]}$ is the restriction of $\lambda_{1}$ to $[a, b]$.

Now assume that $X$ is harmonizable. Recall that the law of $X$ is given by

$$
E e^{i \sum_{k=1}^{p} \xi_{k} X_{t_{k}}}=e^{-b^{\prime} \int_{\mathbb{R}^{N}}\left|\sum_{k=1}^{p} \xi_{k} f\left(t_{k}, \lambda\right)\right|^{\alpha} m(d \lambda)}, b^{\prime} \in(0, \infty) .
$$

Similarly, if we let $F[t,(u, x, v)]=f[t,(u, g(x), v)] h(g(x))^{1 / \alpha} g^{\prime}(x)^{1 / \alpha}, x \in(a, b)$, and $F[t,(u, x, v)]=0$ if $x \notin(a, b)$, then we have a version of $X$ given by

$$
X_{t}=\operatorname{Re} \int_{\mathbb{R}^{N_{1}} \times[a, b] \times \mathbb{R}^{N_{2}}} F(t, s) \zeta_{1}(d s), t \in \mathbb{R}^{N},
$$

where $\zeta_{1}=(n, \Psi)\left(\Psi(\xi)=a^{\prime}\|\xi\|^{\alpha}, \xi \in \mathbb{R}^{2}, a^{\prime} \in(0, \infty)\right)$ is a complex-valued isotropic $\alpha$-stable random measure with control measure $n=\left.m_{1} \otimes \lambda_{1}\right|_{[a, b]} \otimes m_{2}$, where $\left.\lambda_{1}\right|_{[a, b]}$ is the restriction of $\lambda_{1}$ to $[a, b], b=-a \in(0, \infty)$, so that $\left.\lambda_{1}\right|_{[a, b]}$ is symmetric.

Lemma 4.9. Let $X: \mathbb{R}^{N} \rightarrow \mathbb{R}^{d}$ be any symmetric rcll fractional field. Fix an $N$-dimensional compact interval $I=\left[a_{1}, b_{1}\right] \times \cdots \times\left[a_{N}, b_{N}\right] \subset(0, \infty)^{N}$. Assume that $X$ is an $M$-field on $I_{\varepsilon}=\left[a_{1}-\varepsilon, b_{1}+\varepsilon\right] \times \cdots \times\left[a_{N}-\varepsilon, b_{N}+\varepsilon\right] \subset(0, \infty)^{N}$ for some sufficiently small $\varepsilon>0$. Then $X$ is $\mathbf{b}$-related on $I^{\prime}$. Furthermore, if $X$ 
has stationary increments, then $X^{b}$ also has stationary increments, and if $X$ is continuous in probability, then $X^{b}$ also is continuous in probability.

Proof. Write $X_{t}=\int_{\mathbb{R}^{N}} f(t, \lambda) \zeta(d \lambda)$ in the linear case and $X_{t}=\operatorname{Re} \int_{\mathbb{R}^{N}} f(t, \lambda) \zeta(d \lambda)$ in the harmonizable case. Let $D_{h}=[-h, h] \times \mathbb{R}^{N-1}$ for $h>0$. Define $X_{t}^{h}=$ $\int_{D_{h}} f(t, \lambda) \zeta(d \lambda)$ in the linear case and $X_{t}^{h}=\operatorname{Re} \int_{D_{h}} f(t, \lambda) \zeta(d \lambda)$ in the harmonizable case. We note that for $X^{h}$, the control measure $m$ is replaced by $m_{h}$, the restriction of $m$ to $D_{h}$, while $\Psi$ and $\Psi_{r}$ remain unchanged. If $X$ has stationary increments, then $\Psi[(f(s, \lambda)-f(t, \lambda)) \xi]=\Psi[(f(s-t, \lambda)) \xi], \forall s, t, \lambda, \xi$ in the linear case and $\Psi_{r}[f(s, \lambda)-f(t, \lambda), \xi]=\Psi_{r}[f(s-t, \lambda), \xi], \forall s, t, \lambda, \xi$ in the harmonizable case. Therefore, $X^{h}$ has stationary increments if $X$ does. One can also show that replacing control measure $m$ by $m_{h}$ has no effect on continuity in probability. Thus, $X^{h}$ is continuous in probability if $X$ is.

We showed that $X^{h}$ has $\mathbf{b}$ in the proof of Lemma 4.7. It is quite clear that there is a sequence $h_{n} \uparrow \infty$ such that $X^{h_{n}} \stackrel{\text { law }}{\longrightarrow} X$. Denote $X^{h_{n}}$ by $X^{n}$. Fix a compact $F \subset \mathbb{R}^{d}$ and assume that $E\left\{\lambda_{d}\left(X\left(I^{\prime}\right)-F\right)\right\}>0$. By Lemma 4.4, there is $X^{n}$ for some large $n$ such that $E\left\{\lambda_{d}\left(X^{n}\left(I^{\prime}\right)-F\right)\right\}>0$. Since $X$ is symmetric,

$$
\begin{aligned}
& E e^{i \xi \cdot\left(X_{t}-X_{s}\right)}=e^{-\int_{\mathbb{R}^{N}} \Psi\{[f(t, \lambda)-f(s, \lambda)] \xi\} m(d \lambda)} \\
& \leq e^{-\int_{D_{h_{n}}} \Psi\{[f(t, \lambda)-f(s, \lambda)] \xi\} m(d \lambda)} \\
& =E e^{i \xi \cdot\left(X_{t}^{n}-X_{s}^{n}\right)}
\end{aligned}
$$

in the linear case and

$$
\begin{aligned}
& E e^{i \xi \cdot\left(X_{t}-X_{s}\right)}=\exp \left\{-\int_{\mathbb{R}^{N}} \Psi_{r}[f(t, \lambda)-f(s, \lambda), \xi] m(d \lambda)\right\} \\
& \leq \exp \left\{-\int_{D_{h_{n}}} \Psi_{r}[f(t, \lambda)-f(s, \lambda), \xi] m(d \lambda)\right\} \\
& =E e^{i \xi \cdot\left(X_{t}^{n}-X_{s}^{n}\right)}
\end{aligned}
$$

in the harmonizable case. It follows that $Q_{\eta}^{X} \leq Q_{\eta}^{X^{n}}$ for any $\eta \in \mathcal{P}\left(I^{\prime}\right)$. (Comment: In fact we have $Q_{\eta}^{X} \leq Q_{\eta}^{X^{n}}$ for any $\eta \in \mathcal{P}\left(\mathbb{R}_{+}^{N}\right)$.) The proof that $X$ is b-related is complete.

We now direct our attention to the composition of fractional fields with $\tilde{\sigma}^{\alpha}$. This is an important potential-theoretic idea used to compute the Hausdorff dimension of the inverse image of a fractional field.

Lemma 4.10. Let $X: \mathbb{R}^{N} \rightarrow \mathbb{R}^{d}$ be one of $Z^{1}, Z^{2}, Z^{3}$ and $Z^{4}$ as in Lemma 4.7. Then $X \circ \tilde{\sigma}^{\alpha}$ has $\mathbf{b}$, where $\tilde{\sigma}^{\alpha}$ is independent of $X$.

Proof. First assume that $X$ is fractional with a control measure satisfying condition (CS). We do it for linear $X$. The harmonizable counterpart follows suit. Let $X$ be given by (4.47). Let $Y$ be the additive sheet associated with $\zeta$. Let $\Omega_{1}$ be the sample space for $\tilde{\sigma}^{\alpha}$ and $\Omega_{2}$ for $Y$. Fix some $l_{1} \geq|a| \vee|b|$. Let $\mathbf{b}_{2}$ be the $\mathbf{b}$ for $Y$ such that $\mathbf{b}_{2}$ is independent of $Y^{l_{1}}$, which has the domain $\mathbb{R}^{N_{1}} \times\left[-l_{1}, l_{1}\right] \times \mathbb{R}^{N_{2}}$, and $r\left(\mathbf{b}_{2}\left(\omega_{2}\right)\right)=r\left(\omega_{2}\right)$ for each $\omega_{2} \in \Omega_{2}^{\prime} \subset \Omega_{2}$ with $r\left(\Omega_{2}^{\prime}\right)=\left(\Omega_{2}\right)_{l_{1}}$. This is valid by Lemma 4.6. Similarly let $\mathbf{b}_{1}$ be the $\mathbf{b}$ for $\tilde{\sigma}^{\alpha}$ such that $\mathbf{b}_{1}$ is independent of $\left(\tilde{\sigma}^{\alpha}\right)^{l}$, which has the domain $[-l, l)^{N}$, and $r\left(\mathbf{b}_{1}\left(\omega_{1}\right)\right)=r\left(\omega_{1}\right)$ for each $\omega_{1} \in \Omega_{1}^{\prime} \subset \Omega_{1}$ with $r\left(\Omega_{1}^{\prime}\right)=\left(\Omega_{1}\right)_{l}$. $\mathbf{b}_{1}$ exists since $\tilde{\sigma}^{\alpha}$ is an iterated additive process. 
Let $A=\left\{\int_{\mathbb{R}^{N_{1}} \times[a, b] \times \mathbb{R}^{N_{2}}} f\left(\omega_{1}(t), \lambda\right) \zeta^{\omega_{2}}(d \lambda): \omega_{i} \in \Omega_{i}, \omega_{i}(0)=0\right\}$. Then $P(A)=$ 1. The relation $\int_{\mathbb{R}^{N_{1}} \times[a, b] \times \mathbb{R}^{N_{2}}} f\left(\omega_{1}, \lambda\right) \zeta^{\omega_{2}}(d \lambda)=\int_{\mathbb{R}^{N_{1}} \times[a, b] \times \mathbb{R}^{N_{2}}} f\left(\omega_{1}^{\prime}, \lambda\right) \zeta^{\omega_{2}^{\prime}}(d \lambda)$ divides $A$ into disjoint equivalence classes. Denote a class by $C$. Then there exists a well-defined measurable map $\mathbf{b}: A \rightarrow \Omega$ such that there is a

$$
\int_{\mathbb{R}^{N_{1}} \times[a, b] \times \mathbb{R}^{N_{2}}} f\left(\omega_{1}, \lambda\right) \zeta^{\omega_{2}}(d \lambda) \in C
$$

for each class $C$ and for any $\theta^{\prime}=\int_{\mathbb{R}^{N_{1}} \times[a, b] \times \mathbb{R}^{N_{2}}} f\left(\omega_{1}^{\prime}, \lambda\right) \zeta^{\omega_{2}^{\prime}}(d \lambda) \in C, \mathbf{b}\left(\theta^{\prime}\right)=$ $\int_{\mathbb{R}^{N_{1}} \times[a, b] \times \mathbb{R}^{N_{2}}} f\left(\mathbf{b}_{1}\left(\omega_{1}\right), \lambda\right) \zeta^{\mathbf{b}_{2}\left(\omega_{2}\right)}(d \lambda)$ and if $\omega_{i}^{\prime} \in \Omega_{i}^{\prime}$ for all $i$, then $\omega_{i} \in \Omega_{i}^{\prime}$ for all $i$. Since $\mathbf{b}$ is measurable to $\left(\mathbf{b}_{1}, \mathbf{b}_{2}\right)$ and since $X$ and $\tilde{\sigma}^{\alpha}$ are independent, $\mathbf{b}$ is independent of $X^{l}$. Let $A^{\prime}=\left\{\int_{\mathbb{R}^{N_{1}} \times[a, b] \times \mathbb{R}^{N_{2}}} f\left(\omega_{1}(t), \lambda\right) \zeta^{\omega_{2}}(d \lambda): \omega_{i} \in \Omega_{i}^{\prime}, \omega_{i}(0)=\right.$ $0\}$. Clearly, $A^{\prime} \subset A$. Also $r\left(A^{\prime}\right)=r(A)$ because $r_{i}\left(\Omega_{i}^{\prime}\right)=\left(\Omega_{i}\right)_{l}$. For each $\theta^{\prime} \in A^{\prime}$, assuming $\theta^{\prime} \in C$, we have

$$
\begin{gathered}
r\left[\mathbf{b}\left(\theta^{\prime}\right)\right]=\int_{\mathbb{R}^{N_{1}} \times[a, b] \times \mathbb{R}^{N_{2}}} f\left(r\left[\mathbf{b}_{1}\left(\omega_{1}\right)\right], \lambda\right) \zeta^{\mathbf{b}_{2}\left(\omega_{2}\right)}(d \lambda) \\
=\int_{\mathbb{R}^{N_{1}} \times[a, b] \times \mathbb{R}^{N_{2}}} f\left(r\left(\omega_{1}\right), \lambda\right) \zeta^{\omega_{2}}(d \lambda)=\int_{\mathbb{R}^{N_{1}} \times[a, b] \times \mathbb{R}^{N_{2}}} f\left(r\left(\omega_{1}^{\prime}\right), \lambda\right) \zeta^{\omega_{2}^{\prime}}(d \lambda),
\end{gathered}
$$

i.e., $r\left(\mathbf{b}\left(\theta^{\prime}\right)\right)=r\left(\theta^{\prime}\right)$. Note that for $\omega_{2} \in \Omega_{2}^{\prime}$,

$$
\begin{gathered}
\int_{\mathbb{R}^{N_{1}} \times[a, b] \times \mathbb{R}^{N_{2}}}(\cdot) \zeta^{\mathbf{b}_{2}\left(\omega_{2}\right)}(d \lambda) \\
=\int_{\mathbb{R}^{N_{1}} \times[a, b] \times \mathbb{R}^{N_{2}}}(\cdot) \zeta^{r\left(\omega_{2}\right)}(d \lambda)=\int_{\mathbb{R}^{N_{1}} \times[a, b] \times \mathbb{R}^{N_{2}}}(\cdot) \zeta^{\omega_{2}}(d \lambda)
\end{gathered}
$$

since $[a, b] \subset\left[-l_{1}, l_{1}\right]$. The proof that $X \circ \tilde{\sigma}^{\alpha}$ has $\mathbf{b}$ is complete.

Now assume that $X: \mathbb{R}^{N} \rightarrow \mathbb{R}^{d}$ is one of $Z^{1}, Z^{2}, Z^{3}$ and $Z^{4}$ as in Lemma 4.7. We consider only one case. The others are omitted. Set

$$
\begin{aligned}
& X_{t_{1}}^{1}=\int_{\mathbb{R}^{p_{1}} \times\left[a_{1}, b_{1}\right] \times \mathbb{R}^{p_{2}}} f_{1}\left(t_{1}, s\right) \zeta^{1}(d s), t_{1} \in \mathbb{R}^{n_{1}}, \\
& X_{t_{2}}^{2}=\int_{\mathbb{R}^{q_{1}} \times\left[a_{2}, b_{2}\right] \times \mathbb{R}^{q_{2}}} f_{2}\left(t_{2}, s\right) \zeta^{2}(d s), \\
& t_{2} \in \mathbb{R}^{n_{2}}, p_{1}+p_{2}=n_{1}-1, q_{1}+q_{2}=n_{2}-1,
\end{aligned}
$$

where $\zeta^{1}$ and $\zeta^{2}$ are independent. Write $\tilde{\sigma}^{\alpha}=\left(\sigma^{1}, \sigma^{2}\right)$ so that $\sigma^{1}$ takes values in $\mathbb{R}^{n_{1}}$ and $\sigma^{2}$ is $\mathbb{R}^{n_{2}}$-valued. $\sigma_{i}$ are independent additive Lévy processes. Let $Z_{t}^{2}=\left(X_{t_{1}}^{1}, X_{t_{2}}^{2}\right)$ with $t=\left(t_{1}, t_{2}\right)$ and $n_{1}+n_{2}=N$. Then

$$
Z^{2} \circ \tilde{\sigma}_{t}^{\alpha}=\left(\int_{\mathbb{R}^{p_{1}} \times\left[a_{1}, b_{1}\right] \times \mathbb{R}^{p_{2}}} f_{1}\left(\sigma_{t}^{1}, s\right) \zeta^{1}(d s), \int_{\mathbb{R}^{q_{1}} \times\left[a_{2}, b_{2}\right] \times \mathbb{R}^{q_{2}}} f_{2}\left(\sigma_{t}^{2}, s\right) \zeta^{2}(d s)\right) .
$$

Let $Y^{i}$ be the additive sheet associated with $\zeta^{i}$. Note that $Y^{1}, Y^{2}, \sigma^{1}$, and $\sigma^{2}$ are mutually independent. Let $\mathbf{b}_{\sigma^{i}}$ be the $\mathbf{b}$ for $\sigma^{i}$ and $\mathbf{b}_{Y^{i}}$ for $Y^{i}$. One can construct $\mathbf{b}$ for $Z^{2} \circ \tilde{\sigma}^{\alpha}$ with $\mathbf{b}_{\sigma^{1}}, \mathbf{b}_{\sigma^{2}}, \mathbf{b}_{Y^{1}}$ and $\mathbf{b}_{Y^{2}}$ in the same way as for fractional fields given by (4.47). 
We can immediately deduce from Lemma 4.10 that $X \circ \tilde{\sigma}^{\alpha}$ has $\mathbf{b}$, where $X$ is a strictly stable fractional field with a control measure as described in Proposition 4.8. Clearly, the method of proving Proposition 4.5 can be adapted to obtain a deeper result as follows.

Proposition 4.11. Let $\mathbf{x}: \mathbb{R}^{N} \rightarrow \mathbb{R}^{d}$ be any symmetric rcll fractional field. Fix an $N$-dimensional compact interval $I=\left[a_{1}, b_{1}\right] \times \cdots \times\left[a_{N}, b_{N}\right] \subset\left(\mathbb{R}_{i}^{N}\right)^{o}$. Assume that $\mathbf{x}$ is an $M$-field on $I_{\varepsilon}=\left[a_{1}-\varepsilon, b_{1}+\varepsilon\right] \times \cdots \times\left[a_{N}-\varepsilon, b_{N}+\varepsilon\right] \subset\left(\mathbb{R}_{i}^{N}\right)^{o}$ for some sufficiently small $\varepsilon>0$. Then $X=\mathbf{x} \circ \tilde{\sigma}^{\alpha}$ is $\mathbf{b}$-related on $I^{\prime}$, where $\mathbf{x}$ is independent of $\tilde{\sigma}^{\alpha}$. Furthermore, if $\mathbf{x}$ has stationary increments, then $X^{b}$ too has stationary increments.

Here is an interesting example. Plainly a Lévy sheet $X$ is a linear fractional field since $X$ can be given by $X_{t}=\int_{\mathbb{R}^{N}} f(t, \lambda) \zeta(d \lambda), t \in \mathbb{R}^{N}$, where $f(t, \lambda)=1_{Q_{t}}(\lambda)$ and $Q_{t}$ is the rectangle introduced in the definition of additive sheets and has $m=\lambda_{N}$ as a control measure. To see this we notice that $\int_{\mathbb{R}^{N}} \Psi(\xi f(t, \lambda)) m(d \lambda)=$ $m\left(Q_{t}\right) \Psi(\xi), \xi \in \mathbb{R}^{d}$, since $\Psi(0)=0$ and $m\left(Q_{t}\right)<\infty$. Let $X: \mathbb{R}^{N} \rightarrow \mathbb{R}^{d}$ be any symmetric $(N, d)$-Lévy sheet with Lévy exponent $\Psi$. Let $\theta$ be a Lévy process with Lévy exponent $\Psi$. Assume that $\theta$ has a strong Feller resolvent. Under this assumption, $\left(X_{t_{1}}, \cdots, X_{t_{n}}\right)$ has a joint density for any distinct $t_{1}, \cdots, t_{n} \in \mathbb{R}_{o}^{N}$. (Recall that $\mathbb{R}_{o}^{N}=\bigcup_{i}\left(\mathbb{R}_{i}^{N}\right)^{o}$.) It follows from Proposition 4.11 and Lemma 4.10 that $X \circ \tilde{\sigma}^{\alpha}$ is b-related on any $I^{\prime} \subset\left(\mathbb{R}_{i}^{N}\right)^{o}$ and that if $X$ is strictly stable (not necessarily symmetric), $X \circ \tilde{\sigma}^{\alpha}$ has b since $m=\lambda_{N}$ is a control measure of the form assumed in Proposition 4.8.

Markov fields. It is a deep and difficult problem to determine $\mathbf{b}$ for an arbitrary Markov field. However, it is clear that if a field $X$ is adapted to an additive field or more generally to an additive sheet, then $X$ has $\mathbf{b}$. While the adaptability issue is certainly of great interest, such a method for Markov fields has not been found in the literature. On the other hand, by standard martingale argument in general a (one-parameter) nonexplosive Markov process is adapted to an additive process in one way or another. Thus, under some mild conditions, one can construct b for a Markov process. For instance, homogeneous Itô SDEs driven by Lévy processes, continuous diffusions, and homogeneous jump diffusions always have $\mathbf{b}$, and Feller processes satisfying an analytic condition (weak spatial homogeneity) have b. All these Markov processes are adapted to Lévy processes. (Consider a concrete example here: The elementary Ornstein-Uhlenbeck process in $\mathbb{R}^{d}: Y_{t}=$ $e^{-t Q} y+\int_{0}^{t} e^{(s-t) Q} d X_{s}, y \in \mathbb{R}^{d}$, where $Q$ is a $d \times d$ matrix whose eigenvalues have positive real parts and $X$ is a Lévy process in $\mathbb{R}^{d}$, is a Feller process. Write $Y_{t}=Y_{t}^{1}+Y_{t}^{2}$, where $Y_{t}^{1}=e^{-t Q} y$ is a continuous deterministic process and $Y_{t}^{2}=$ $\int_{0}^{t} e^{(s-t) Q} d X_{s}$. Clearly $Y^{2}$ is adapted to $X$ and therefore $Y^{2}$ has $\mathbf{b}$. Since $Y^{1}$ has $\mathbf{b}$ (trivial) and since $Y^{1}$ and $Y^{2}$ are independent, $Y$ has $\mathbf{b}$ by Lemma 2.1 ; cf. $Z^{3}$.) It follows that Markov fields of either type of

$$
\left(X_{t_{1}}^{1}, \cdots, X_{t_{N}}^{N}\right), \quad X_{t_{1}}^{1}+\cdots+X_{t_{N}}^{N}
$$

have $\mathbf{b}$, where $X^{j}$ are independent Markov processes each of which has $\mathbf{b}$, by Lemma 2.1 ; cf. $Z^{2}$ and $Z^{4}$. 


\section{REFERENCES}

[1] R. M. Blumenthal and R. K. Getoor, Markov processes and potential theory, Pure and Applied Mathematics, Vol. 29, Academic Press, New York, 1968. MR0264757 (41 \#9348)

[2] Kai Lai Chung, Lectures from Markov processes to Brownian motion, Grundlehren der Mathematischen Wissenschaften [Fundamental Principles of Mathematical Science], vol. 249, Springer-Verlag, New York, 1982. MR648601 (84c:60091)

[3] Robert C. Dalang and Eulalia Nualart, Potential theory for hyperbolic SPDEs, Ann. Probab. 32 (2004), no. 3A, 2099-2148, DOI 10.1214/009117904000000685. MR2073187(2005f:60163)

[4] P. J. Fitzsimmons and Thomas S. Salisbury, Capacity and energy for multiparameter Markov processes (English, with French summary), Ann. Inst. H. Poincaré Probab. Statist. 25 (1989), no. 3, 325-350. MR,1023955 (91d:60180)

[5] John Hawkes, Potential theory of Lévy processes, Proc. London Math. Soc. (3) 38 (1979), no. 2, 335-352, DOI 10.1112/plms/s3-38.2.335. MR.531166 (80g:60077)

[6] Davar Khoshnevisan and Zhan Shi, Brownian sheet and capacity, Ann. Probab. 27 (1999), no. 3, 1135-1159, DOI 10.1214/aop/1022677442. MR1733143 (2002f:60150)

Department of Mathematics, Tulane University, New Orleans, Louisiana 70118

E-mail address: myang1968@yahoo.com 